\title{
STRONG CONVERGENCE TO THE HOMOGENIZED LIMIT OF PARABOLIC EQUATIONS WITH RANDOM COEFFICIENTS
}

\author{
JOSEPH G. CONLON AND ARASH FAHIM
}

\begin{abstract}
This paper is concerned with the study of solutions to discrete parabolic equations in divergence form with random coefficients and their convergence to solutions of a homogenized equation. It has previously been shown that if the random environment is translational invariant and ergodic, then solutions of the random equation converge under diffusive scaling to solutions of a homogenized parabolic PDE. In this paper point-wise estimates are obtained on the difference between the averaged solution to the random equation and the solution to the homogenized equation for certain random environments which are strongly mixing.
\end{abstract}

\section{INTRODUCTION}

Let $(\Omega, \mathcal{F}, P)$ be a probability space and denote by $\langle\cdot\rangle$ expectation w.r. to the measure $P$. We assume that the $d$ dimensional integer lattice $\mathbf{Z}^{d}$ acts on $\Omega$ by space translation operators $\tau_{x, 0}: \Omega \rightarrow \Omega, x \in \mathbf{Z}^{d}$, which are measure preserving and satisfy the properties $\tau_{x, 0} \tau_{y, 0}=\tau_{x+y, 0}, \tau_{0,0}=$ identity, $x, y \in \mathbf{Z}^{d}$. We assume also that either the integers $\mathbf{Z}$ or the real line $\mathbf{R}$ acts on $\Omega$ by time translation operators $\tau_{0, t}: \Omega \rightarrow \Omega$, where $t \in \mathbf{Z}$ in the former case and $t \in \mathbf{R}$ in the latter. In either case we assume that for all $t, s$, one has $\tau_{0, t} \tau_{0, s}=\tau_{0, t+s}$ and that the operators $\tau_{0, t}$ commute with the operators $\tau_{x, 0}$, so we may set $\tau_{x, t}=\tau_{x, 0} \tau_{0, t}=\tau_{0, t} \tau_{x, 0}$.

Consider a bounded measurable function $\mathbf{a}: \Omega \rightarrow \mathbf{R}^{d(d+1) / 2}$ from $\Omega$ to the space of symmetric $d \times d$ matrices which satisfies the quadratic form inequality

$$
\lambda I_{d} \leq \mathbf{a}(\omega) \leq \Lambda I_{d}, \quad \omega \in \Omega,
$$

where $I_{d}$ is the identity matrix in $d$ dimensions and $\Lambda, \lambda$ are positive constants. In the case when $\mathbf{Z}$ acts on $\Omega$ by operators $\tau_{0, t}$, we shall be interested in solutions $u(x, t, \omega)$ to the discrete parabolic equation

(1.2) $u(x, t+1, \omega)-u(x, t, \omega)=-\nabla^{*} \mathbf{a}\left(\tau_{x, t} \omega\right) \nabla u(x, t, \omega), \quad x \in \mathbf{Z}^{d}, t \geq 0, \omega \in \Omega$,

with initial data

$$
u(x, 0, \omega)=h(x), \quad x \in \mathbf{Z}^{d}, \omega \in \Omega .
$$

In the case when $\mathbf{R}$ acts on $\Omega$ by operators $\tau_{0, t}$, we shall be interested in solutions $u(x, t, \omega)$ to the corresponding continuous in time, discrete in space parabolic

Received by the editors March 23, 2012 and, in revised form, November 1, 2012. 2010 Mathematics Subject Classification. Primary 81T08, 82B20, 35R60, 60J75.

Key words and phrases. Euclidean field theory, PDE with random coefficients, homogenization. 
equation

$$
\frac{\partial u(x, t, \omega)}{\partial t}=-\nabla^{*} \mathbf{a}\left(\tau_{x, t} \omega\right) \nabla u(x, t, \omega), \quad x \in \mathbf{Z}^{d}, t \geq 0, \omega \in \Omega,
$$

with initial data (1.3). In (1.2) and (1.4) we take $\nabla$ to be the discrete gradient operator, which has adjoint $\nabla^{*}$. Thus $\nabla$ is a $d$ dimensional column operator and $\nabla^{*}$ a $d$ dimensional row operator which act on functions $\phi: \mathbf{Z}^{d} \rightarrow \mathbf{R}$ by

$$
\begin{array}{rll}
\nabla \phi(x) & =\left(\nabla_{1} \phi(x), \ldots, \nabla_{d} \phi(x)\right), & \nabla_{i} \phi(x)=\phi\left(x+\mathbf{e}_{i}\right)-\phi(x), \\
\nabla^{*} \phi(x) & =\left(\nabla_{1}^{*} \phi(x), \ldots, \nabla_{d}^{*} \phi(x)\right), & \nabla_{i}^{*} \phi(x)=\phi\left(x-\mathbf{e}_{i}\right)-\phi(x) .
\end{array}
$$

In (1.5) the vector $\mathbf{e}_{i} \in \mathbf{Z}^{d}$ has 1 as the $i$ th coordinate and 0 for the other coordinates, $1 \leq i \leq d$.

One expects that if the translation operators $\tau_{x, t}$ are ergodic on $\Omega$, then solutions to the random equation (1.2) or (1.4) converge to solutions of a constant coefficient homogenized equation under diffusive scaling. Thus suppose $f: \mathbf{R}^{d} \rightarrow \mathbf{R}$ is a $C^{\infty}$ function with compact support, and for $\varepsilon$ satisfying $0<\varepsilon \leq 1$ set $h(x)=f(\varepsilon x), x \in$ $\mathbf{Z}^{d}$, in (1.3), and let $u_{\varepsilon}(x, t, \omega)$ denote the corresponding solution to (1.2) or (1.4) with this initial data. It has been shown in [21, just assuming ergodicity of the translation operators, that $u_{\varepsilon}\left(x / \varepsilon, t / \varepsilon^{2}, \omega\right)$ converges in probability as $\varepsilon \rightarrow 0$ to a function $u_{\text {hom }}(x, t), x \in \mathbf{R}^{d}, t>0$, which is the solution to a constant coefficient parabolic PDE

$$
\frac{\partial u_{\mathrm{hom}}(x, t)}{\partial t}=-\nabla^{*} \mathbf{a}_{\mathrm{hom}} \nabla u_{\mathrm{hom}}(x, t), \quad x \in \mathbf{R}^{d}, t>0
$$

with initial condition

$$
u_{\text {hom }}(x, 0)=f(x), \quad x \in \mathbf{R}^{d} .
$$

The $d \times d$ symmetric matrix $\mathbf{a}_{\text {hom }}$ in (1.6) satisfies the quadratic form inequality (1.11). Similar results under various ergodic type assumptions on $\Omega$ can be found in 1, 6, 12, 28. In time-independent environments the corresponding results for elliptic equations in divergence form have been proven much earlier; see [19,20, 26, 33].

In this paper we shall confine ourselves to studying the expectation $\langle u(x, t, \cdot)\rangle$ of the solution $u(x, t, \omega)$ to (1.2) and (1.4) with initial condition (1.3). Our first theorem is consistent with the result of [21] already mentioned:

Theorem 1.1. Let $f: \mathbf{R}^{d} \rightarrow \mathbf{R}$ be a $C^{\infty}$ function of compact support and set $h(x)=f(\varepsilon x), x \in \mathbf{Z}^{d}$, in (1.3). For the translation group $\tau_{x, t}, x \in \mathbf{Z}^{d}, t \in \mathbf{Z}$, on $\Omega$ assume that one of the operators $\tau_{\mathbf{e}_{j}, 0}, j=1, \ldots, d$, or $\tau_{0,1}$ is ergodic on $(\Omega, \mathcal{F}, P)$. Then if $4 d \Lambda \leq 1$ the solution $u_{\varepsilon}(x, t, \omega)$ of (1.2) with initial data (1.3) has the property

$$
\lim _{\varepsilon \rightarrow 0} \sup _{x \in \varepsilon \mathbf{Z}^{d}, t \in \varepsilon^{2} \mathbf{Z}^{+}}\left|\left\langle u_{\varepsilon}\left(x / \varepsilon, t / \varepsilon^{2}, \cdot\right)\right\rangle-u_{\text {hom }}(x, t)\right|=0 .
$$

For the translation group $\tau_{x, t}, x \in \mathbf{Z}^{d}, t \in \mathbf{R}$, on $\Omega$ assume that one of the operators $\tau_{\mathbf{e}_{j}, 0}, j=1, \ldots, d$, or the continuous 1 parameter group $\tau_{0, t}, t \in \mathbf{R}$, is ergodic on $(\Omega, \mathcal{F}, P)$. Then the solution $u_{\varepsilon}(x, t, \omega)$ of (1.4) with initial data (1.3) has the property

$$
\lim _{\varepsilon \rightarrow 0} \sup _{x \in \varepsilon \mathbf{Z}^{d}, t>0}\left|\left\langle u_{\varepsilon}\left(x / \varepsilon, t / \varepsilon^{2}, \cdot\right)\right\rangle-u_{\text {hom }}(x, t)\right|=0 .
$$


It has been shown in the case of homogenization of elliptic equations in divergence form with random coefficients that a rate of convergence in homogenization can be obtained provided the random environment satisfies some quantitative strong mixing property. The first results in this direction were proven in the 1980's by Yurinski [32, but there have been several papers more recently extending his work. In particular, Caffarelli and Souganidis [10 have obtained rates of convergence results in homogenization of fully nonlinear PDE. In recent work of Gloria and Otto [15] an optimal rate of convergence result was obtained for linear elliptic equations in divergence form. Following an idea of Naddaf and Spencer [24], they express the quantitative strong mixing assumption as a Poincaré inequality. This formulation of the strong mixing assumption is very useful when the random environment is a Euclidean field theory with uniformly convex Lagrangian.

For the case of parabolic equations in divergence form with random coefficients, we shall obtain a rate of convergence, but only for the averaged solution to the parabolic equation, as in Theorem 1.1, and for two particular environments. For the discrete time problem (1.2), (1.3) we assume the environment is i.i.d. That is, we assume the variables $\mathbf{a}\left(\tau_{x, t^{*}}\right),(x, t) \in \mathbf{Z}^{d+1}$, are i.i.d. For the continuous time problem (1.3), (1.4) we assume the environment is the stationary process associated with a massive Euclidean field theory. The only paper we were able to find in the literature proving results on rate of convergence in homogenization for the parabolic problem is the recent paper [23], in which the environment is assumed fixed in time. In [23] as in the present paper, the results are restricted to establishing rates of convergence for the mean $\left\langle u_{\varepsilon}\left(x / \varepsilon, t / \varepsilon^{2}, \cdot\right)\right\rangle$ of the solution of the parabolic equation with random coefficients to $u_{\text {hom }}(x, t)$.

The Euclidean field theory is determined by a potential $V: \mathbf{R}^{d} \rightarrow \mathbf{R}$, which is a $C^{2}$ uniformly convex function, and a mass $m>0$. Thus the second derivative $\mathbf{a}(\cdot)=V^{\prime \prime}(\cdot)$ of $V(\cdot)$ is assumed to satisfy the inequality (1.1). Consider functions $\phi: \mathbf{Z}^{d} \times \mathbf{R} \rightarrow \mathbf{R}$, which we denote as $\phi(x, t)$ where $x$ lies on the integer lattice $\mathbf{Z}^{d}$ and $t$ on the real line $\mathbf{R}$. Let $\Omega$ be the space of all such functions which have the property that for each $x \in \mathbf{Z}^{d}$ the function $t \rightarrow \phi(x, t)$ on $\mathbf{R}$ is continuous, and let $\mathcal{F}$ be the Borel algebra generated by finite dimensional rectangles $\{\phi(\cdot, \cdot) \in \Omega$ : $\left.\left|\phi\left(x_{i}, t_{i}\right)-a_{i}\right|<r_{i}, i=1, \ldots, N\right\}$, where $\left(x_{i}, t_{i}\right) \in \mathbf{Z}^{d} \times \mathbf{R}, a_{i} \in \mathbf{R}, r_{i}>0, i=$ $1, \ldots, N, N \geq 1$. The translation operators $\tau_{x, t}: \Omega \rightarrow \Omega,(x, t) \in \mathbf{Z}^{d} \times \mathbf{R}$, are defined by $\tau_{x, t} \phi(z, s)=\phi(x+z, t+s), z \in \mathbf{Z}^{d}, s \in \mathbf{R}$.

For any $d \geq 1$ and $m>0$ one can define [7,14] a unique ergodic translation invariant probability measure $P$ on $(\Omega, \mathcal{F})$ which depends on the function $V$ and $m$. In this measure the variables $\phi(x, t), x \in \mathbf{Z}^{d}, t>0$, conditioned on the variables $\phi(x, 0), x \in \mathbf{Z}^{d}$, are determined as solutions of the infinite dimensional stochastic differential equation

$$
d \phi(x, t)=-\frac{\partial}{\partial \phi(x, t)} \sum_{x^{\prime} \in \mathbf{Z}^{d}} \frac{1}{2}\left\{V\left(\nabla \phi\left(x^{\prime}, t\right)\right)+m^{2} \phi\left(x^{\prime}, t\right)^{2} / 2\right\} d t+d B(x, t)
$$

where $x \in \mathbf{Z}^{d}$ and $t>0$, and where $B(x, \cdot), x \in \mathbf{Z}^{d}$, are independent copies of Brownian motion. Formally the invariant measure for the Markov process (1.10) is the Euclidean field theory measure

$$
\exp \left[-\sum_{x \in \mathbf{Z}^{d}} V(\nabla \phi(x))+m^{2} \phi(x)^{2} / 2\right] \prod_{x \in \mathbf{Z}^{d}} d \phi(x) / \text { normalization. }
$$


Hence if the variables $\phi(x, 0), x \in \mathbf{Z}^{d}$, have distribution determined by (1.11), then $\phi(\cdot, t), t>0$, is a stationary process and so can be extended to all $t \in \mathbf{R}$ to yield a measure $P$ on $(\Omega, \mathcal{F})$. For this measure the translation operators $\tau_{x, t},(x, t) \in$ $\mathbf{Z}^{d} \times \mathbf{R}$, form a group of measure preserving transformations on $(\Omega, \mathcal{F}, P)$.

Theorem 1.2. Let $f: \mathbf{R}^{d} \rightarrow \mathbf{R}$ be a $C^{\infty}$ function of compact support and set $h(x)=f(\varepsilon x), x \in \mathbf{Z}^{d}$, in (1.3). Then if $4 d \Lambda \leq 1$ and the variables $\mathbf{a}\left(\tau_{x, t}\right),(x, t) \in$ $\mathbf{Z}^{d+1}$, are i.i.d., the solution $u_{\varepsilon}(x, t, \omega)$ of (1.2) with initial data (1.3) has the property

$$
\sup _{x \in \varepsilon \mathbf{Z}^{d}, t \in \varepsilon^{2} \mathbf{Z}^{+}}\left|\left\langle u_{\varepsilon}\left(x / \varepsilon, t / \varepsilon^{2}, \cdot\right)\right\rangle-u_{\text {hom }}(x, t)\right| \leq C \varepsilon^{\alpha}, \quad 0<\varepsilon \leq 1,
$$

where $\alpha>0$ is a constant depending only on $d, \Lambda / \lambda$ and $C$ is a constant depending only on $d, \Lambda, \lambda$ and the function $f(\cdot)$.

Let $\tilde{\mathbf{a}}: \mathbf{R} \rightarrow \mathbf{R}^{d(d+1) / 2}$ be a $C^{1}$ function on $\mathbf{R}$ with values in the space of symmetric $d \times d$ matrices which satisfy the quadratic form inequality (1.1). Let $(\Omega, \mathcal{F}, P)$ be the probability space of fields $\phi(\cdot, \cdot)$ determined by (1.10), (1.11), and set $\mathbf{a}(\cdot)$ in (1.4) to be $\mathbf{a}(\phi)=\tilde{\mathbf{a}}(\phi(0,0)), \phi \in \Omega$. Suppose in addition that the derivative D $\tilde{\mathbf{a}}(\cdot)$ of $\tilde{\mathbf{a}}(\cdot)$ satisfies the inequality $\|D \tilde{\mathbf{a}}(\cdot)\|_{\infty} \leq \Lambda_{1}$. Then the solution $u_{\varepsilon}(x, t, \omega)$ of (1.4) with initial data (1.3) has the property

$$
\sup _{x \in \varepsilon \mathbf{Z}^{d}, t>0}\left|\left\langle u_{\varepsilon}\left(x / \varepsilon, t / \varepsilon^{2}, \cdot\right)\right\rangle-u_{\mathrm{hom}}(x, t)\right| \leq C \varepsilon^{\alpha}, \quad 0<\varepsilon \leq 1,
$$

where $\alpha>0$ is a constant depending only on $d, \Lambda / \lambda$ and $C$ is a constant depending only on $d, \Lambda, \lambda, m, \Lambda_{1}$ and the function $f(\cdot)$.

Remark 1. It is clear that the exponent $\alpha>0$ in Theorem 1.2 must satisfy $\alpha \leq 2$. This follows from the fact that the error in approximating the solution to the heat equation on $\mathbf{R}^{d}$ by the solution to the corresponding lattice problem on $\varepsilon \mathbf{Z}^{d}$ is $O\left(\varepsilon^{2}\right)$. One can conclude from our method of proof that if $\lambda / \Lambda$ is sufficiently close to 1 , then the exponent $\alpha$ can be taken equal to 1 . The exponent $\alpha$ can be taken equal to 2 for $\lambda / \Lambda$ sufficiently close to 1 provided $\nabla$ in (1.2), (1.4) is defined by central difference rather than forward difference as in (1.5) and $\mathbf{a}(\cdot)$ is a diagonal matrix. In addition the environment $(\Omega, \mathcal{F}, P)$ must satisfy a reflection invariant condition. The i.i.d. environment satisfies this reflection invariance condition, but the massive field theory environment determined by (1.10) satisfies it only if the function $V: \mathbf{R}^{d} \rightarrow \mathbf{R}$ is reflection invariant (see Appendix).

We consider what Theorem 1.2 tells us about the expectation of Green's function for the equations (1.2) and (1.4). By translation invariance of the measure we have that

$$
\langle u(x, t, \cdot)\rangle=\sum_{y \in \mathbf{Z}^{d}} G_{\mathbf{a}}(x-y, t) h(y), \quad x \in \mathbf{Z}^{d},
$$

where $G_{\mathbf{a}}(x, t)$ is the expected value of Green's function. Setting $h(x)=f(\varepsilon x), x \in$ $\mathbf{Z}^{d}$, then (1.14) may be written as

$$
\left\langle u_{\varepsilon}\left(x / \varepsilon, t / \varepsilon^{2}, \cdot\right)\right\rangle=\int_{\varepsilon Z^{d}} \varepsilon^{-d} G_{\mathbf{a}}\left(\frac{x-z}{\varepsilon}, \frac{t}{\varepsilon^{2}}\right) f(z) d z, \quad x \in \varepsilon \mathbf{Z}^{d},
$$

where integration over $\varepsilon \mathbf{Z}^{d}$ is defined by

$$
\int_{\varepsilon Z^{d}} g(z) d z=\sum_{z \in \varepsilon \mathbf{Z}^{d}} g(z) \varepsilon^{d} .
$$


Let $G_{\mathbf{a}_{\text {hom }}}(x, t), x \in \mathbf{R}^{d}, t>0$, be Green's function for the PDE (1.6). One easily sees that $G_{\mathbf{a}_{\text {hom }}}(\cdot, \cdot)$ satisfies the scaling property

$$
\varepsilon^{-d} G_{\mathbf{a}_{\mathrm{hom}}}\left(x / \varepsilon, t / \varepsilon^{2}\right)=G_{\mathbf{a}_{\mathrm{hom}}}(x, t), \quad \varepsilon>0, x \in \mathbf{R}^{d}, t>0 .
$$

Hence Theorem 1.2 implies that averages of $\varepsilon^{-d} G_{\mathbf{a}}\left(x / \varepsilon, t / \varepsilon^{2}\right)-\varepsilon^{-d} G_{\mathbf{a}_{\text {hom }}}\left(x / \varepsilon, t / \varepsilon^{2}\right)$ with respect to $x \in \varepsilon \mathbf{Z}^{d}$ are bounded by $C \varepsilon^{\alpha}$ for some constant $C$. Conversely Theorem 1.2 is implied by the point-wise estimate on Green's functions:

$$
\begin{aligned}
& \left|\varepsilon^{-d} G_{\mathbf{a}}\left(x / \varepsilon, t / \varepsilon^{2}\right)-\varepsilon^{-d} G_{\mathbf{a}_{\mathrm{hom}}}\left(x / \varepsilon, t / \varepsilon^{2}\right)\right| \\
& \quad \leq \frac{C \varepsilon^{\alpha}}{\left[\Lambda t+\varepsilon^{2}\right]^{(d+\alpha) / 2}} \exp \left[-\gamma \min \left\{\frac{|x|}{\varepsilon}, \frac{|x|^{2}}{\Lambda t+\varepsilon^{2}}\right\}\right],
\end{aligned}
$$

provided $\Lambda t \geq \varepsilon^{2}$ and $x \in \varepsilon \mathbf{Z}^{d}$.

It is clear that the inequality (1.18) for $\varepsilon<1$ follows from the same inequality for $\varepsilon=1$ :

$$
\left|G_{\mathbf{a}}(x, t)-G_{\mathbf{a}_{\mathrm{hom}}}(x, t)\right| \leq \frac{C}{[\Lambda t+1]^{(d+\alpha) / 2}} \exp \left[-\gamma \min \left\{|x|, \frac{|x|^{2}}{\Lambda t+1}\right\}\right]
$$

provided $\Lambda t \geq 1$ and $x \in \mathbf{Z}^{d}$. We shall prove such an inequality and also similar inequalities for the derivatives of the expectation of Green's function,

$$
\left|\nabla G_{\mathbf{a}}(x, t)-\nabla G_{\mathbf{a}_{\mathrm{hom}}}(x, t)\right| \leq \frac{C}{[\Lambda t+1]^{(d+1+\alpha) / 2}} \exp \left[-\gamma \min \left\{|x|, \frac{|x|^{2}}{\Lambda t+1}\right\}\right],
$$

$$
\left|\nabla \nabla G_{\mathbf{a}}(x, t)-\nabla \nabla G_{\mathbf{a}_{\mathrm{hom}}}(x, t)\right| \leq \frac{C}{[\Lambda t+1]^{(d+2+\alpha) / 2}} \exp \left[-\gamma \min \left\{|x|, \frac{|x|^{2}}{\Lambda t+1}\right\}\right] \text {. }
$$

Theorem 1.3. Let $(\Omega, \mathcal{F}, P)$ and $\mathbf{a}(\omega), \omega \in \Omega$, be as in the statement of Theorem 1.2. Then for $d \geq 1$ there exists $\alpha, \gamma>0$ depending only on $d$ and $\Lambda / \lambda$, such that (1.19), (1.20) and (1.21) hold for some positive constant $C$. In the discrete time case, $C$ depends only on $d, \Lambda, \lambda$, and in the continuous time case, only on $d, \lambda, \Lambda, m, \Lambda_{1}$.

The proofs of Theorem 1.2 and Theorem 1.3 follow the same lines as the proofs of the corresponding results for elliptic equations proved in [10. One begins with a Fourier representation for the average of the solution to the random parabolic equation, which was obtained in 8 . Then for the i.i.d. environment the generalization by Jones [18] of the Calderon-Zygmund theorem [5] to parabolic multipliers, together with some interpolation inequalities, yields Theorem 1.2 and the inequalities (1.19), (1.20) of Theorem 1.3 in the discrete time case. Similarly to [10 we need to use the result of Delmotte and Deuschel [1] on the Hölder continuity of the second difference $\nabla \nabla G_{\mathbf{a}}(x, t)$ in order to prove (1.21). In the continuous time case we need in addition to prove some Poincaré inequalities for time-dependent fields. To do this we follow the methodology of Gourcy-Wu [16] by using the Clark-Ocone formula [25].

\section{FOURIER SPACE REPRESENTATION AND HOMOGENIZATION}

In this section we shall prove the homogenization result Theorem 1.1. The proof of this is based on a Fourier representation for the solutions of (1.2), (1.4), which was given in 8 . 
We begin by summarizing relevant results from [8] for the discrete time equation (1.2). Thus we are assuming a probability space $(\Omega, \mathcal{F}, P)$ and a set of translation operators $\tau_{x, t}, x \in \mathbf{Z}^{d}, t \in \mathbf{Z}$, acting on $\Omega$. For $\xi \in \mathbf{R}^{d}$ and $\psi: \Omega \rightarrow \mathbf{C}$ a measurable function we define the $\xi$ derivative of $\psi(\cdot)$ in the $j$ direction $\partial_{j, \xi}$ and its adjoint $\partial_{j, \xi}^{*}$ by

$$
\begin{aligned}
& \partial_{j, \xi} \psi(\omega)=e^{-i \mathbf{e}_{j} \cdot \xi} \psi\left(\tau_{\mathbf{e}_{j}, 0} \omega\right)-\psi(\omega), \\
& \partial_{j, \xi}^{*} \psi(\omega)=e^{i \mathbf{e}_{j} \cdot \xi} \psi\left(\tau_{-\mathbf{e}_{j}, 0} \omega\right)-\psi(\omega) .
\end{aligned}
$$

The $d$ dimensional column $\xi$ derivative operator $\partial_{\xi}$ is then given by $\partial_{\xi}=$ $\left(\partial_{1, \xi}, \ldots, \partial_{d, \xi}\right)$. Similarly to (1.5) its adjoint $\partial_{\xi}^{*}$ is given by the row operator $\partial_{\xi}^{*}=\left(\partial_{1, \xi}^{*}, \ldots, \partial_{d, \xi}^{*}\right)$. Let $P: L^{2}(\Omega) \rightarrow L^{2}(\Omega)$ be the projection orthogonal to the constant function, and $\eta \in \mathbf{C}$ with real part be denoted by $\Re \eta$ and imaginary part by $\Im \eta$ so that $\eta=\Re \eta+i \Im \eta$. Then for $\xi \in \mathbf{R}^{d}$ and $\eta \in \mathbf{C}$ with $\Re \eta>0$ there is a unique square integrable solution $\Phi(\xi, \eta, \omega)$ to the equation

$$
e^{\eta} \Phi\left(\xi, \eta, \tau_{0,1} \omega\right)-\Phi(\xi, \eta, \omega)+P \partial_{\xi}^{*} \mathbf{a}(\omega) \partial_{\xi} \Phi(\xi, \eta, \omega)=-P \partial_{\xi}^{*} \mathbf{a}(\omega),
$$

provided $4 d \Lambda \leq 1$. Thus there is a unique row vector $\Phi(\xi, \eta, \omega)=\left[\Phi_{1}(\xi, \eta, \omega), \ldots\right.$, $\left.\Phi_{d}(\xi, \eta, \omega)\right]$ with $\Phi_{j}(\xi, \eta, \cdot) \in L^{2}(\Omega), j=1, \ldots, d$, which satisfies (2.2). Let $q(\xi, \eta)=$ $\left[q_{r, r^{\prime}}(\xi, \eta)\right]$ be the $d \times d$ matrix function given in terms of the solution to (2.2) by the formula

$$
q(\xi, \eta)=\langle\mathbf{a}(\cdot)\rangle+\left\langle\mathbf{a}(\cdot) \partial_{\xi} \Phi(\xi, \eta, \cdot)\right\rangle .
$$

One can easily see that the homogenized diffusion constant $\mathbf{a}_{\text {hom }}$ of (1.6) in the case of an ergodic environment $(\Omega, \mathcal{F}, P)$ is given by the formula $\mathbf{a}_{\text {hom }}=\lim _{\eta \rightarrow 0} q(0, \eta)$. This follows by observing that the solution to (2.2) in the case $\xi=0, \Re \eta>0$ is also the solution to the equation

$$
e^{\eta} \Phi\left(0, \eta, \tau_{0,1} \omega\right)-\Phi(\xi, \eta, \omega)+\partial_{0}^{*} \mathbf{a}(\omega) \partial_{0} \Phi(\xi, \eta, \omega)=-\partial_{0}^{*} \mathbf{a}(\omega),
$$

since $\langle\Phi(0, \eta, \cdot)\rangle=0$. The standard formula for $\mathbf{a}_{\text {hom }}$ is given by $\mathbf{a}_{\text {hom }}=$ $\lim _{\eta \rightarrow 0} q(0, \eta)$, where $\Phi(0, \eta, \omega)$ is the solution to (2.4); see [15, 32 for the elliptic case $\tau_{0,1} \equiv$ identity and 21] for the parabolic case.

We define the $d$ dimensional periodic column vector $e(\xi) \in \mathbf{C}^{d}$ to have $j$ th entry given by the formula $e_{j}(\xi)=e^{-i \mathbf{e}_{j} \cdot \xi}-1,1 \leq j \leq d$. It was shown in 8 , that the solution $u(x, t, \omega), x \in \mathbf{Z}^{d}, t=0,1,2, \ldots, \omega \in \Omega$, to the initial value problem (1.2), (1.3) has the representation

$$
\begin{aligned}
& u(x, t, \omega) \\
& \quad=\frac{1}{(2 \pi)^{d+1}} \int_{[-\pi, \pi]^{d+1}} \frac{\hat{h}(\xi) e^{-i \xi \cdot x+\eta(t+1)}}{e^{\eta}-1+e(\xi)^{*} q(\xi, \eta) e(\xi)}\left[1+\Phi\left(\xi, \eta, \tau_{x, t} \omega\right) e(\xi)\right] d[\Im \eta] d \xi,
\end{aligned}
$$

where $\hat{h}(\cdot)$ is the Fourier transform of $h(\cdot)$,

$$
\hat{h}(\xi)=\sum_{x \in \mathbf{Z}^{d}} h(x) e^{i \xi \cdot x} .
$$

Note that the integration in (2.5) with respect to $\Im \eta$ over the interval $[-\pi, \pi]$ is for any fixed value of $\Re \eta>0$. Since the integrand is a function of $\eta$ which is analytic in $\eta$ for $\Re \eta>0$ and also periodic of period $2 \pi$ with respect to $\Im \eta$, the integral on 
the RHS of (2.5) is independent of $\Re \eta>0$. It follows from (2.5) that the FourierLaplace transform $\hat{G}_{\mathbf{a}}(\xi, \eta)$ of the averaged Green's function $G_{\mathbf{a}}(\cdot, \cdot)$ for (1.2), (1.3) given by

$$
\hat{G}_{\mathbf{a}}(\xi, \eta)=\sum_{t=0}^{\infty} \sum_{x \in \mathbf{Z}^{d}} G_{\mathbf{a}}(x, t) \exp [i x . \xi-\eta t], \quad \xi \in \mathbf{R}^{d}, \Re \eta>0,
$$

has the representation

$$
\hat{G}_{\mathbf{a}}(\xi, \eta)=e^{\eta} /\left[e^{\eta}-1+e(\xi)^{*} q(\xi, \eta) e(\xi)\right] .
$$

The solution to (2.5) can be generated by a convergent perturbation expansion. Let $\mathcal{H}(\Omega)$ be the Hilbert space of measurable functions $\psi: \Omega \rightarrow \mathbf{C}^{d}$ with norm $\|\psi\|$ given by $\|\psi\|^{2}=\left\langle|\psi(\cdot)|^{2}\right\rangle$. We define an operator $T_{\xi, \eta}$ on $\mathcal{H}(\Omega)$ as follows: For any $g \in \mathcal{H}$, let $\psi(\xi, \eta, \omega)$ be the solution to the equation

$$
\frac{1}{\Lambda}\left[e^{\eta} \psi\left(\xi, \eta, \tau_{0,1} \omega\right)-\psi(\xi, \eta, \omega)\right]+\partial_{\xi}^{*} \partial_{\xi} \psi(\xi, \eta, \omega)=\partial_{\xi}^{*} g(\omega) .
$$

Then $T_{\xi, \eta} g(\cdot)=\partial_{\xi} \psi(\xi, \eta, \cdot)$, or more explicitly

$$
T_{\xi, \eta} g(\omega)=\Lambda \sum_{t=0}^{\infty} e^{-\eta(t+1)} \sum_{x \in \mathbf{Z}^{d}}\left\{\nabla \nabla^{*} G_{\Lambda}(x, t)\right\}^{*} \exp [-i x . \xi] g\left(\tau_{x,-t-1} \omega\right),
$$

where $G_{\Lambda}(\cdot)$ is the solution to the initial value problem

$$
\begin{array}{r}
G_{\Lambda}(x, t+1)-G_{\Lambda}(x, t)+\Lambda \nabla^{*} \nabla G_{\Lambda}(x, t)=0, \quad x \in \mathbf{Z}^{d}, t \in \mathbf{Z}, t \geq 0 \\
G_{\Lambda}(x, 0)=\delta(x), \quad x \in \mathbf{Z}^{d} .
\end{array}
$$

Equation (2.11) has a unique solution provided $4 d \Lambda \leq 1$, and the function $G_{\Lambda}(x, t)$ satisfies an inequality

$$
\begin{aligned}
0 \leq G_{\Lambda}(x, t)+(\Lambda t+1)^{1 / 2}\left|\nabla G_{\Lambda}(x, t)\right|+(\Lambda t+1)\left|\nabla \nabla^{*} G_{\Lambda}(x, t)\right| \\
\leq \frac{C_{d}}{[\Lambda t+1]^{d / 2}} \exp \left[-\frac{\min \left\{|x|,|x|^{2} /(\Lambda t+1)\right\}}{C_{d}}\right],
\end{aligned}
$$

where $C_{d}>0$ is a constant depending only on dimension $d$. The inequality (2.12) can be proved using the Fourier inversion formula and contour integration, since the Fourier transform of $G_{\Lambda}$ can be explicitly computed from (2.11).

The operator $T_{\xi, \eta}$ is bounded on $\mathcal{H}(\Omega)$ with $\left\|T_{\xi, \eta}\right\| \leq 1$, provided $\xi \in \mathbf{R}^{d}, \Re \eta>0$. Now on setting $\mathbf{a}(\cdot)=\Lambda\left[I_{d}-\mathbf{b}(\cdot)\right]$, one sees that (2.2) is equivalent to the equation

$$
\partial_{\xi} \Phi(\xi, \eta, \cdot)=P T_{\xi, \eta}\left[\mathbf{b}(\cdot) \partial_{\xi} \Phi(\xi, \eta, \cdot)\right]+P T_{\xi, \eta}[\mathbf{b}(\cdot)] .
$$

Since $\left\|T_{\xi, \eta}\right\| \leq 1$ and $\|\mathbf{b}(\omega)\| \leq 1-\lambda / \Lambda, \omega \in \Omega$, the Neumann series for the solution to 2.13 ) given by

$$
\partial_{\xi} \Phi(\xi, \eta, \cdot)=\sum_{m=1}^{\infty}\left[P T_{\xi, \eta} \mathbf{b}(\cdot)\right]^{m}
$$

converges in $\mathcal{H}(\Omega)$.

It will be useful later to express the operator $T_{\xi, \eta}$ in its Fourier representation. To do this we use the standard notation for the Fourier transform of a function 
$h: \mathbf{Z}^{d+1} \rightarrow \mathbf{C}$ which we denote by $h(x, t), x \in \mathbf{Z}^{d}, t \in \mathbf{Z}$. Letting $\hat{h}(\zeta, \theta), \quad \zeta \in$ $[-\pi, \pi]^{d}, \theta \in[-\pi, \pi]$, be the Fourier transform of $h(\cdot, \cdot)$, then

$$
\hat{h}(\zeta, \theta)=\sum_{x \in \mathbf{Z}^{d}} \sum_{t \in \mathbf{Z}} h(x, t) e^{i x \cdot \zeta+i t \cdot \theta} .
$$

The Fourier inversion formula yields

$$
h(x, t)=\frac{1}{(2 \pi)^{d+1}} \int_{[-\pi, \pi]^{d+1}} \hat{h}(\zeta, \theta) e^{-i x . \zeta-i t \theta} d \zeta d \theta, \quad x \in \mathbf{Z}^{d}, t \in \mathbf{Z} .
$$

Now the action of the translation group $\tau_{x, 0}, x \in \mathbf{Z}^{d}$, on $\Omega$ can be described by a set $A_{1}, \ldots, A_{d}$ of commuting self-adjoint operators on $L^{2}(\Omega)$ so that

$$
f\left(\tau_{x, 0} \cdot\right)=\exp [i x . A] f(\cdot), \quad x \in \mathbf{Z}^{d}, f \in L^{2}(\Omega),
$$

where $A=\left(A_{1}, \ldots, A_{d}\right)$. Similarly the action of the translation group $\tau_{0, t}, t \in \mathbf{Z}$, on $\Omega$ can be described by a self-adjoint operator $B$ on $L^{2}(\Omega)$ which commutes with $A_{1}, \ldots, A_{d}$ so that

$$
f\left(\tau_{0, t^{*}}\right)=\exp [-i t B] f(\cdot), \quad t \in \mathbf{Z}, f \in L^{2}(\Omega) .
$$

It follows then from (2.9), (2.17), (2.18) that

$$
T_{\xi, \eta} g(\cdot)=\frac{\Lambda e(\xi-A) e^{*}(\xi-A)}{e^{\eta-i B}-1+\Lambda e(\xi-A)^{*} e(\xi-A)} g(\cdot) .
$$

The Neumann series (2.14) for the solution to (2.13) yields a convergent perturbation expansion for the function $q(\xi, \eta)$ of (2.3). Thus for $m=1,2, \ldots$, let the matrix function $h_{m}(\xi, \eta)$ be defined for $\Re \eta>0, \xi \in \mathbf{R}^{d}$, by

$$
h_{m}(\xi, \eta)=\left\langle\mathbf{b}(\cdot)\left[P T_{\xi, \eta} \mathbf{b}(\cdot)\right]^{m}\right\rangle ;
$$

whence (2.3), (2.14) imply that

$$
q(\xi, \eta)=\langle\mathbf{a}(\cdot)\rangle-\Lambda \sum_{m=1}^{\infty} h_{m}(\xi, \eta) .
$$

It is easy to see that the function $q(\xi, \eta)$ is $C^{\infty}$ for $\xi \in \mathbf{R}^{d}, \Re \eta>0$. As in [8, 10] we can extend this result as follows:

Proposition 2.1. Suppose that $4 d \Lambda \leq 1$ and any of the translation operators $\tau_{\mathbf{e}_{j}, 0}, 1 \leq j \leq d$, or $\tau_{0,1}$ is ergodic on $\Omega$. Then the limit $\lim _{(\xi, \eta) \rightarrow(0,0)} q(\xi, \eta)=$ $q(0,0)$ exists. If any of the translation operators is weak mixing [27] on $\Omega$, then $q(\xi, \eta), \xi \in \mathbf{R}^{d}, \Re \eta>0$, extends to a continuous function on $\xi \in \mathbf{R}^{d}, \Re \eta \geq 0$.

Proof. We follow the same argument as in Lemma 2.5 of 8 and Proposition 2.1 of [10.

Remark 2. Note that the projection operator $P$ in equation (2.2) plays a critical role in establishing continuity. For a constant function $g(\cdot) \equiv v \in \mathbf{C}^{d}$, one has

$$
T_{\xi, \eta} g(\cdot)=\left[e(\xi)^{*} v\right] e(\xi) /\left[\left(e^{\eta}-1\right) / \Lambda+e(\xi)^{*} e(\xi)\right],
$$

which does not extend to a continuous function of $(\xi, \eta)$ on the set $\xi \in \mathbf{R}^{d}, \Re \eta \geq 0$.

Next we show that the function $q(\xi, \eta)$ with domain $\xi \in \mathbf{R}^{d}, \Re \eta>0$, can be extended to complex $\xi=\Re \xi+i \Im \xi \in \mathbf{C}^{d}$ with small imaginary part. 
Lemma 2.1. The $C^{\infty}$ operator valued function $(\xi, \eta) \rightarrow T_{\xi, \eta}$ with domain $\{(\xi, \eta)$ : $\left.\xi \in \mathbf{R}^{d}, \Re \eta>0\right\}$ and range the space of bounded linear operators $\mathcal{B}[\mathcal{H}(\Omega)]$ on $\mathcal{H}(\Omega)$ has an analytic continuation to a region $\left\{(\xi, \eta) \in \mathbf{C}^{d+1}: 0<\Re \eta<\Lambda,|\Im \xi|<\right.$ $\left.C_{1} \sqrt{\Re \eta / \Lambda}\right\}$, where $C_{1}$ is a constant depending only on $d$. For $(\xi, \eta)$ in this region the norm of $T_{\xi, \eta}$ satisfies the inequality $\left\|T_{\xi, \eta}\right\| \leq 1+C_{2}|\Im \xi|^{2} /[\Re \eta / \Lambda]$, where the constant $C_{2}$ depends only on $d$.

Proof. That there is an analytic continuation to the region $\left\{\xi \in \mathbf{C}^{d}: 0<\Re \eta<\right.$ $\left.\Lambda,|\Im \xi|<C_{1} \sqrt{\Re \eta / \Lambda}\right\}$ follows from (2.10), (2.12) using the fact that $\left|\nabla \nabla^{*} G_{\Lambda}(x, t)\right|$ is bounded by $(\Lambda t+1)^{-1}$ times the RHS of (2.12). For $(\xi, \eta)$ in this region one has that

$$
\Re[-\eta(t+1)-i x \cdot \xi] \leq \sup _{|\theta|<1}\left[-\theta^{2} \Lambda(t+1)+C_{1} \theta|x|\right] \leq \min \left\{C_{1}|x|, C_{1}^{2}|x|^{2} / 4 \Lambda(t+1)\right\} .
$$

Hence using the representation (2.10) for $T_{\xi, \eta}$, we see that the analytic continuation extends to any region $\left\{\xi \in \mathbf{C}^{d}: 0<\Re \eta<\Lambda,|\Im \xi|<C_{1} \sqrt{\Re \eta / \Lambda}\right\}$ provided $C_{1}$ satisfies the inequalities $C_{1}<C_{d}^{-1}, C_{1}^{2}<4 C_{d}^{-1}$, where $C_{d}$ is the constant in (2.12).

The bound on $\left\|T_{\xi, \eta}\right\|$ can be obtained from (2.9). Thus on multiplying (2.9) by $\bar{\psi}\left(\xi, \eta, \tau_{0,1} \omega\right)$ we see that

$$
\begin{aligned}
\frac{e^{\Re \eta}}{\Lambda}\left\langle|\psi(\xi, \eta, \cdot)|^{2}\right\rangle \leq & \frac{1}{\Lambda}\left|\left\langle\bar{\psi}\left(\xi, \eta, \tau_{0,1} \cdot\right)\left[I-\Lambda \partial_{\xi}^{*} \partial_{\xi}\right] \psi(\xi, \eta, \cdot)\right\rangle\right| \\
& +\left|\left\langle\bar{\psi}\left(\xi, \eta, \tau_{0,1} \cdot\right) \partial_{\xi}^{*} g(\cdot)\right\rangle\right| .
\end{aligned}
$$

Since $4 d \Lambda \leq 1$ it follows that for $\xi \in \mathbf{R}^{d}$ the operator $I-\Lambda \partial_{\xi}^{*} \partial_{\xi}$ is symmetric non-negative definite. Hence if $\xi \in \mathbf{R}^{d}$ one has that

$$
\begin{aligned}
\left|\left\langle\bar{\psi}\left(\xi, \eta, \tau_{0,1} \cdot\right)\left[I-\Lambda \partial_{\xi}^{*} \partial_{\xi}\right] \psi(\xi, \eta, \cdot)\right\rangle\right| \\
\leq \frac{1}{2}\left\langle\bar{\psi}\left(\xi, \eta, \tau_{0,1} \cdot\right)\left[I-\Lambda \partial_{\xi}^{*} \partial_{\xi}\right] \psi\left(\xi, \eta, \tau_{0,1} \cdot\right)\right\rangle \\
\quad+\frac{1}{2}\left\langle\bar{\psi}(\xi, \eta, \cdot)\left[I-\Lambda \partial_{\xi}^{*} \partial_{\xi}\right] \psi(\xi, \eta, \cdot)\right\rangle .
\end{aligned}
$$

Similarly one has that

$$
\begin{aligned}
\left|\left\langle\bar{\psi}\left(\xi, \eta, \tau_{0,1} \cdot\right) \partial_{\xi}^{*} g(\cdot)\right\rangle\right| & \\
\leq & \frac{1}{2}\left\langle\bar{\psi}\left(\xi, \eta, \tau_{0,1} \cdot\right)\left[\partial_{\xi}^{*} \partial_{\xi}\right] \psi\left(\xi, \eta, \tau_{0,1} \cdot\right)\right\rangle \\
& +\frac{1}{2}\|g(\cdot)\|^{2} .
\end{aligned}
$$

We conclude from (2.24) -2.26 that $\left\|T_{\xi, \eta}\right\| \leq 1$ provided $\xi \in \mathbf{R}^{d}$ and $\Re \eta>0$. This argument can then be extended as in Lemma 2.1 of $\left[10\right.$ to $\xi \in \mathbf{C}^{d}$.

Corollary 2.1. The $d \times d$ matrix function $q(\xi, \eta)$ with domain $\{(\xi, \eta): \xi \in$ $\left.\mathbf{R}^{d}, \Re \eta>0\right\}$ has an analytic continuation to a region $\left\{\xi \in \mathbf{C}^{d}: 0<\Re \eta<\right.$ $\left.\Lambda,|\Im \xi|<C_{1} \sqrt{\lambda \Re \eta / \Lambda^{2}}\right\}$, where $C_{1}$ is a constant depending only on d. There is a constant $C_{2}$ depending only on d such that for $\xi$ in this region,

$$
\|q(\xi, \eta)-q(\Re \xi, \eta)\| \leq \frac{C_{2} \Lambda^{2}}{\lambda} \frac{|\Im \xi|}{\sqrt{\Re \eta / \Lambda}} .
$$


Proof. The fact that $q(\xi, \eta)$ has an analytic continuation follows from the representations (2.20), (2.21), Lemma 2.1 and the matrix norm bound $\|\mathbf{b}(\omega)\| \leq$ $1-\lambda / \Lambda, \omega \in \Omega$. On summing the perturbation series (2.21), we conclude that for $\xi$ satisfying $|\Im \xi|<C_{1} \sqrt{\lambda \Re \eta / \Lambda^{2}}$, then $\|q(\xi, \eta)\| \leq C_{2} \Lambda^{2} / \lambda$ for a constant $C_{2}$ depending only on $d$, provided $C_{1}$ is chosen sufficiently small, depending only on $d$. By arguing as in Lemma 2.1 we also see that there are positive constants $C_{1}, C_{2}$ such that

$$
\left\|T_{\xi, \eta}-T_{\Re \xi, \eta}\right\| \leq C_{2}|\Im \xi| / \sqrt{\Re \eta / \Lambda}, \quad \xi \in \mathbf{C}^{d},|\Im \xi|<C_{1} \sqrt{\Re \eta / \Lambda} .
$$

The inequality (2.27) follows from (2.28).

It follows from Corollary 2.1 that for $\xi \in \mathbf{C}^{d}, \eta \in \mathbf{C}$ with fixed $\Im \xi \in \mathbf{R}^{d}, \Re \eta>$ 0 satisfying $0<\Re \eta<\Lambda,|\Im \xi|<C_{1} \sqrt{\lambda \Re \eta / \Lambda^{2}}$, the periodic matrix function $(\Re \xi, \Im \eta) \rightarrow q(\xi, \eta)$ on $\mathbf{R}^{d+1}$ with fundamental region $[-\pi, \pi]^{d+1}$ is bounded.

Corollary 2.2. There exist positive constants $C_{1}, C_{2}$ depending only on $d$ and $\Lambda / \lambda$ such that

$$
\left|e^{\eta}-1+e(\bar{\xi})^{*} q(\xi, \eta) e(\xi)\right| \geq C_{2}\left[|\eta|+\Lambda|e(\Re \xi)|^{2}\right],
$$

provided $0<\Re \eta<\Lambda,|\Im \xi|<C_{1} \sqrt{\Re \eta / \Lambda}$.

Proof. The inequality (2.29) follows from Corollary 2.1 and Lemma 2.7, Lemma 2.8 of 8 .

Proof of Theorem 1.1 (Discrete time case). Taking $h(x)=f(\varepsilon x)$, we have from (2.5) that

$$
\begin{aligned}
& \left\langle u_{\varepsilon}\left(x / \varepsilon, t / \varepsilon^{2}, \cdot\right)\right\rangle \\
& \quad=\frac{1}{(2 \pi)^{d+1}} \int_{[-\pi / \varepsilon, \pi / \varepsilon]^{d}} \int_{-\pi / \varepsilon^{2}}^{\pi / \varepsilon^{2}} \frac{\varepsilon^{2} \hat{f}_{\varepsilon}(\xi) e^{-i \xi \cdot x+\eta\left(t+\varepsilon^{2}\right)}}{e^{\varepsilon^{2} \eta}-1+e(\varepsilon \xi)^{*} q\left(\varepsilon \xi, \varepsilon^{2} \eta\right) e(\varepsilon \xi)} d[\Im \eta] d \xi,
\end{aligned}
$$

where

$$
\hat{f}_{\varepsilon}(\xi)=\sum_{y \in \varepsilon \mathbf{Z}^{d}} \varepsilon^{d} f(y) e^{i y \cdot \xi}
$$

We also have that

$$
u_{\mathrm{hom}}(x, t)=\frac{1}{(2 \pi)^{d+1}} \int_{\mathbf{R}^{d}} \int_{\mathbf{R}} \frac{\hat{f}(\xi) e^{-i \xi \cdot x+\eta t}}{\eta+\xi^{*} q(0,0) \xi} d[\Im \eta] d \xi,
$$

where $\hat{f}(\cdot)$ is the Fourier transform of $f(\cdot)$,

$$
\hat{f}(\xi)=\int_{\mathbf{R}^{d}} f(y) e^{i y \cdot \xi} d y, \quad \xi \in \mathbf{R}^{d} .
$$

Since $f: \mathbf{R}^{d} \rightarrow \mathbf{R}$ is $C^{\infty}$ of compact support it follows from (2.31), (2.33) that

$$
\begin{gathered}
\sup _{0<\varepsilon \leq 1, \xi \in[-\pi / \varepsilon, \pi / \varepsilon]^{d}}\left|\hat{f}_{\varepsilon}(\xi)\right|\left(1+|\xi|^{2}\right)^{N}<\infty, \\
\sup _{0<\varepsilon \leq 1, \xi \in[-\pi / \varepsilon, \pi / \varepsilon]^{d}}\left|\hat{f}_{\varepsilon}(\xi)-\hat{f}(\xi)\right|\left(1+|\xi|^{2}\right)^{N} / \varepsilon^{2}<\infty,
\end{gathered}
$$

where $N$ in (2.34) can be arbitrarily large. 
We first observe from (2.34) and Lemma 2.9 and Lemma 2.10 of 8 that

$$
\int_{|\xi|>1 / \sqrt{\Lambda \varepsilon}}\left|\int_{-\pi / \varepsilon^{2}}^{\pi / \varepsilon^{2}} \frac{\varepsilon^{2} \hat{f}_{\varepsilon}(\xi) e^{-i \xi \cdot x+\eta\left(t+\varepsilon^{2}\right)}}{e^{\varepsilon^{2} \eta}-1+e(\varepsilon \xi)^{*} q\left(\varepsilon \xi, \varepsilon^{2} \eta\right) e(\varepsilon \xi)} d[\Im \eta]\right| d \xi \leq C \varepsilon,
$$

for a constant $C$ depending only on the function $f(\cdot)$ and $d, \lambda, \Lambda$. Since the function $q(\xi, \eta)$ is continuous at $(\xi, \eta)=(0,0)$, we similarly see there exists for any $\delta>0$ an $\varepsilon(\delta)>0$ depending only on $\delta, d, \lambda, \Lambda$ such that if $\varepsilon \leq \varepsilon(\delta)$, then

$\left|\int_{-\pi / \varepsilon^{2}}^{\pi / \varepsilon^{2}} \frac{\varepsilon^{2} e^{\eta\left(t+\varepsilon^{2}\right)}}{e^{\varepsilon^{2} \eta}-1+e(\varepsilon \xi)^{*} q\left(\varepsilon \xi, \varepsilon^{2} \eta\right) e(\varepsilon \xi)}-\frac{\varepsilon^{2} e^{\eta\left(t+\varepsilon^{2}\right)}}{e^{\varepsilon^{2} \eta}-1+e(\varepsilon \xi)^{*} q(0,0) e(\varepsilon \xi)} d[\Im \eta]\right| \leq \delta$

for all $\xi \in[-\pi / \varepsilon, \pi / \varepsilon]^{d}$ such that $|\xi| \leq 1 / \sqrt{\Lambda \varepsilon}$. It follows from (2.34), (2.35), (2.36) that for any $\delta>0$ there exists $\varepsilon(\delta)>0$ depending only on $\delta, d, \lambda, \Lambda$, and the function $f(\cdot)$ such that

$$
\begin{aligned}
\sup _{x \in \varepsilon \mathbf{Z}^{d}, t \in \varepsilon^{2} \mathbf{Z}^{+}} & \mid\left\langle u_{\varepsilon}\left(x / \varepsilon, t / \varepsilon^{2}, \cdot\right)\right\rangle \\
& \quad-\int_{[-\pi / \varepsilon, \pi / \varepsilon]^{d}} \int_{-\pi / \varepsilon^{2}}^{\pi / \varepsilon^{2}} \frac{\varepsilon^{2} \hat{f}(\xi) e^{-i \xi \cdot x+\eta\left(t+\varepsilon^{2}\right)}}{e^{\varepsilon^{2} \eta}-1+e(\varepsilon \xi)^{*} q(0,0) e(\varepsilon \xi)} d[\Im \eta] d \xi \mid \leq \delta
\end{aligned}
$$

provided $\varepsilon \leq \varepsilon(\delta)$. If we use the identities

$$
\frac{1}{2 \pi} \int_{-\pi / \varepsilon^{2}}^{\pi / \varepsilon^{2}} \frac{\varepsilon^{2} e^{\eta\left(t+\varepsilon^{2}\right)}}{e^{\varepsilon^{2} \eta}-1+e(\varepsilon \xi)^{*} q(0,0) e(\varepsilon \xi)} d[\Im \eta]=\left[1-e(\varepsilon \xi)^{*} q(0,0) e(\varepsilon \xi)\right]^{t / \varepsilon^{2}},
$$

$$
\frac{1}{2 \pi} \int_{\mathbf{R}} \frac{e^{\eta t}}{\eta+\xi^{*} q(0,0) \xi} d[\Im \eta]=\exp \left[-\left\{\xi^{*} q(0,0) \xi\right\} t\right],
$$

we can conclude from (2.32), (2.37) that for any $\delta>0$ there exists $\varepsilon(\delta)>0$ depending only on $\delta, d, \lambda, \Lambda$, and the function $f(\cdot)$ such that

$$
\sup _{x \in \varepsilon \mathbf{Z}^{d}, t \in \varepsilon^{2} \mathbf{Z}^{+}}\left|\left\langle u_{\varepsilon}\left(x / \varepsilon, t / \varepsilon^{2}, \cdot\right)\right\rangle-u_{\text {hom }}(x, t)\right| \leq \delta,
$$

provided $\varepsilon \leq \varepsilon(\delta)$.

Remark 3 . It is easy to see that if $\tau_{\mathbf{e}_{j}, 0}$ for some $j, 1 \leq j \leq d$, or $\tau_{0,1}$ acts ergodically on $\Omega$, then

$$
\lim _{(\xi, \eta) \rightarrow(0,0)}\left[e^{\Re \eta}-1\right]\|\Phi(\xi, \eta, \cdot)\|^{2}=0 .
$$

In the case of an elliptic equation with random coefficients, the limit corresponding to (2.41) implies that the solution to the random equation converges in distribution to the solution of the homogenized equation [10]. This is not the case for the parabolic problem due to the fact that the integrand in (2.30), when multiplied by an arbitrary bounded function of $\eta$, can have a logarithmic divergence upon integration with respect to $\Im \eta$. 
In the continuous time case there is a similar development to the above. The solution $u(x, t, \omega)$ to (1.3), (1.4) has the representation

$$
\begin{aligned}
& u(x, t, \omega) \\
& \quad=\frac{1}{(2 \pi)^{d+1}} \int_{[-\pi, \pi]^{d}} \int_{-\infty}^{\infty} \frac{\hat{h}(\xi) e^{-i \xi \cdot x+\eta t}}{\eta+e(\xi)^{*} q(\xi, \eta) e(\xi)}\left[1+\Phi\left(\xi, \eta, \tau_{x, t} \omega\right) e(\xi)\right] d[\Im \eta] d \xi,
\end{aligned}
$$

where now the $d$ dimensional row vector $\Phi(\xi, \eta, \omega)$ is the solution to the equation

$$
\eta \Phi(\xi, \eta, \omega)+\partial \Phi(\xi, \eta, \omega)+P \partial_{\xi}^{*} \mathbf{a}(\omega) \partial_{\xi} \Phi(\xi, \eta, \omega)=-P \partial_{\xi}^{*} \mathbf{a}(\omega) .
$$

In (2.43) the operator $\partial$ is the infinitesimal generator of the time translation group $\tau_{0, t}, t \in \mathbf{R}$. The $d \times d$ matrix function $q(\xi, \eta)$ in (2.42) is given in terms of the solution to (2.43) by the formula (2.3). It follows from (2.42) that the Fourier transform $\hat{G}_{\mathbf{a}}(\xi, \eta)$ of the averaged Green's function $G_{\mathbf{a}}(\cdot, \cdot)$ for (1.4) defined by

$$
\hat{G}_{\mathbf{a}}(\xi, \eta)=\int_{0}^{\infty} d t \sum_{x \in \mathbf{Z}^{d}} G_{\mathbf{a}}(x, t) \exp [i x \cdot \xi-\eta t]
$$

has the representation

$$
\hat{G}_{\mathbf{a}}(\xi, \eta)=1 /\left[\eta+e(\xi)^{*} q(\xi, \eta) e(\xi)\right], \quad \xi \in \mathbf{R}^{d}, \Re \eta>0 .
$$

Let $G(x, t), x \in \mathbf{Z}^{d}, t>0$, be the solution to the initial value problem

$$
\begin{aligned}
\frac{\partial G(x, t)}{\partial t}+\nabla^{*} \nabla G(x, t) & =0, \quad x \in \mathbf{Z}^{d}, t>0, \\
G(x, 0) & =\delta(x), \quad x \in \mathbf{Z}^{d} .
\end{aligned}
$$

Then the equation (2.43) is equivalent to (2.13), where the operator $T_{\xi, \eta}$ is given by the formula

$$
T_{\xi, \eta} g(\omega)=\Lambda \int_{0}^{\infty} e^{-\eta t} d t \sum_{x \in \mathbf{Z}^{d}}\left\{\nabla \nabla^{*} G_{\Lambda}(x, t)\right\}^{*} \exp [-i x . \xi] g\left(\tau_{x,-t} \omega\right),
$$

with $G_{\Lambda}(x, t)=G(x, \Lambda t), x \in \mathbf{Z}^{d}, t>0$. Note that in the continuous time case there is no restriction on the value of $\Lambda>0$. The operator $T_{\xi, \eta}$ of (2.47) is bounded on $\mathcal{H}(\Omega)$ with $\left\|T_{\xi, \eta}\right\| \leq 1$, provided $\xi \in \mathbf{R}^{d}, \Re \eta>0$, and hence the Neumann series for the solution of (2.13) converges in $\mathcal{H}(\Omega)$.

As in the discrete time case it will be useful later to express the operator $T_{\xi, \eta}$ in its Fourier representation. To do this we use the standard notation for the Fourier transform of a function $h: \mathbf{Z}^{d} \times \mathbf{R} \rightarrow \mathbf{C}$, which we denote by $h(x, t), x \in \mathbf{Z}^{d}, t \in \mathbf{R}$. Letting $\hat{h}(\zeta, \theta), \zeta \in[-\pi, \pi]^{d}, \theta \in \mathbf{R}$, be the Fourier transform of $h(\cdot, \cdot)$, then

$$
\hat{h}(\zeta, \theta)=\int_{-\infty}^{\infty} d t \sum_{x \in \mathbf{Z}^{d}} h(x, t) e^{i x \cdot \zeta+i t \theta} .
$$

The Fourier inversion formula yields

$$
h(x, t)=\frac{1}{(2 \pi)^{d+1}} \int_{-\infty}^{\infty} \int_{[-\pi, \pi]^{d}} \hat{h}(\zeta, \theta) e^{-i x \cdot \zeta-i t \theta} d \zeta d \theta, \quad x \in \mathbf{Z}^{d}, t \in \mathbf{R}
$$


Now the action of the translation group $\tau_{x, 0}, x \in \mathbf{Z}^{d}$, on $\Omega$ can be described by a set $A_{1}, \ldots, A_{d}$ of commuting self-adjoint operators on $L^{2}(\Omega)$ so that

$$
f\left(\tau_{x, 0}\right)=\exp [i x . A] f(\cdot), \quad x \in \mathbf{Z}^{d}, f \in L^{2}(\Omega),
$$

where $A=\left(A_{1}, \ldots, A_{d}\right)$. Similarly the action of the translation group $\tau_{0, t}, t \in \mathbf{R}$, on $\Omega$ can be described by a self-adjoint operator $B$ on $L^{2}(\Omega)$ which commutes with $A_{1}, \ldots, A_{d}$ so that

$$
f\left(\tau_{0, t^{*}}\right)=\exp [-i t B] f(\cdot), \quad t \in \mathbf{R}, f \in L^{2}(\Omega),
$$

whence the infinitesimal generator $\partial$ in (2.43) is given by $\partial=-i B$. It follows now from (2.47), (2.50), 2.51) that

$$
T_{\xi, \eta} g(\cdot)=\frac{\Lambda e(\xi-A) e^{*}(\xi-A)}{\eta-i B+\Lambda e(\xi-A)^{*} e(\xi-A)} g(\cdot) .
$$

The Neumann series for the solution to (2.13), with the operator $T_{\xi, \eta}$ given now by (2.52), yields a convergent perturbation expansion (2.20), (2.21) for the function $q(\xi, \eta)$. It is easy to see that the analogues of Proposition 2.1, Lemma 2.1 and Corollary 2.1 continue to hold for the continuous time case. In the continuous time analogue of Corollary 2.2 the inequality (2.29) is replaced by

$$
\left|\eta+e(\bar{\xi})^{*} q(\xi, \eta) e(\xi)\right| \geq C\left[|\eta|+\Lambda|e(\Re \xi)|^{2}\right] .
$$

The inequality (2.53) follows from Lemma 5.3 of [8].

Proof of Theorem 1.1 (Continuous time case). We proceed as in the discrete time case replacing (2.5) by (2.42) and using Lemma 5.4 and Lemma 5.5 of [8] in place of Lemma 2.9 and Lemma 2.10 of [8].

\section{RATE OF CONVERGENCE IN HOMOGENIZATION}

In this section we shall prove Theorem 1.2 under the assumption that the solutions $\Phi(\xi, \eta, \omega)$ of (2.2), (2.43) satisfy a certain property which we describe below. In $\S 5$ we shall show that this property holds for the independent variable environment, and in $\S 6$ for the massive field theory environment. We first consider the discrete time case, whence $\Phi(\xi, \eta, \omega)$ is a solution to (2.2).

For $1 \leq p \leq \infty$ let $L^{p}\left(\mathbf{Z}^{d+1}, \mathbf{C}^{d} \otimes \mathbf{C}^{d}\right)$ be the Banach space of $d \times d$ matrix valued functions $g: \mathbf{Z}^{d+1} \rightarrow \mathbf{C}^{d} \otimes \mathbf{C}^{d}$ with norm $\|g\|_{p}$ defined by

$$
\begin{aligned}
\|g\|_{p}^{p} & =\sup _{v \in \mathbf{C}^{d}:|v|=1} \sum_{(x, t) \in \mathbf{Z}^{d+1}}|g(x, t) v|^{p} \text { if } p<\infty, \\
\|g\|_{\infty} & =\sup _{v \in \mathbf{C}^{d}:|v|=1}\left[\sup _{(x, t) \in \mathbf{Z}^{d+1}}|g(x, t) v|\right],
\end{aligned}
$$

where $|g(x, t) v|$ is the Euclidean norm of the vector $g(x, t) v \in \mathbf{C}^{d}$. We assume the following:

Hypothesis 3.1. There exists $p_{0}(\Lambda / \lambda)>1$ depending only on $d, \Lambda / \lambda$ and a constant $C$ such that for $1 \leq p \leq p_{0}(\Lambda / \lambda)$,

$$
\left\|P \sum_{(x, t) \in \mathbf{Z}^{d+1}} g(x, t) \mathbf{b}\left(\tau_{x,-t^{*}}\right)\left[v+\partial_{\xi} \Phi\left(\xi, \eta, \tau_{x,-t^{*}}\right) v\right]\right\| \leq C\|g\|_{p}|v|
$$

for all $\xi \in \mathbf{R}^{d}, \eta \in \mathbf{C}$ with $0<\Re \eta<\Lambda$, and $g \in L^{p}\left(\mathbf{Z}^{d+1}, \mathbf{C}^{d} \otimes \mathbf{C}^{d}\right), v \in \mathbf{C}^{d}$. 
Remark 4. Note from Lemma 2.3 of [8] that since $\left\|\partial_{\xi} \Phi(\xi, \eta, \cdot) v\right\|^{2} \leq \Lambda|v|^{2} / \lambda$ for $\xi \in \mathbf{R}^{d}, \Re \eta>0$, the inequality (3.2) holds for $p=1$. Hence if (3.2) holds for $p=p_{0}(\Lambda / \lambda)$, by the Riesz convexity theorem [31] it also holds for any $p$ satisfying $1 \leq p \leq p_{0}(\Lambda / \lambda)$.

We show that if Hypothesis 3.1 holds, then the function $q(\xi, \eta)$ defined by (2.3) is Hölder continuous with exponent depending on $d, \Lambda / \lambda$.

Lemma 3.1. Assume Hypothesis 3.1 holds. Then there exists $\alpha>0$ depending only on $d, \Lambda / \lambda$ and a constant $C_{\alpha}$ such that the $d \times d$ matrix function $q(\xi, \eta)$ of (2.3) satisfies the inequality

$$
\left\|q\left(\xi^{\prime}, \eta^{\prime}\right)-q(\xi, \eta)\right\| \leq C_{\alpha} \Lambda\left[\left|\xi^{\prime}-\xi\right|^{\alpha}+\left|\left(\eta^{\prime}-\eta\right) / \Lambda\right|^{\alpha / 2}\right]
$$

for all $\xi^{\prime}, \xi \in \mathbf{R}^{d}, 0<\Re \eta^{\prime}, \Re \eta<\Lambda$.

Proof. It follows from (2.20) that

$h_{k}\left(\xi^{\prime}, \eta^{\prime}\right)-h_{k}(\xi, \eta)=\sum_{j=1}^{k}\left\langle\mathbf{b}(\cdot)\left[P T_{\xi^{\prime}, \eta^{\prime}} \mathbf{b}(\cdot)\right]^{j-1} P\left[T_{\xi^{\prime}, \eta^{\prime}}-T_{\xi, \eta}\right] \mathbf{b}(\cdot)\left[P T_{\xi, \eta} \mathbf{b}(\cdot)\right]^{k-j}\right\rangle$.

Hence we conclude from (2.13), (2.21) and (3.4) upon using the inequality $\left\|T_{\xi^{\prime}, \eta^{\prime}}\right\| \leq$ 1 that for $v \in \mathbf{C}^{d}$,

$$
\left\|\left[q\left(\xi^{\prime}, \eta^{\prime}\right)-q(\xi, \eta)\right] v\right\| \leq\left(\Lambda^{2} / \lambda\right)\left\|P\left[T_{\xi^{\prime}, \eta^{\prime}}-T_{\xi, \eta}\right] \mathbf{b}(\cdot)\left[v+\partial_{\xi} \Phi(\xi, \eta, \cdot) v\right]\right\| .
$$

From (2.10) we see that the RHS of (3.5) is the same as the LHS of (3.2) with the function $g(\cdot, \cdot)$ given by the formula

$$
g(x, t)=\Lambda\left[\nabla \nabla^{*} G_{\Lambda}(x, t-1)\right]^{*}\left[e^{-\eta^{\prime} t-i x \cdot \xi^{\prime}}-e^{-\eta t-i x \cdot \xi}\right] .
$$

Observe now that for $0 \leq \alpha \leq 1$ one has

$$
\begin{aligned}
& \left|e^{-\eta^{\prime} t-i x \cdot \xi^{\prime}}-e^{-\eta t-i x \cdot \xi}\right| \\
& \quad \leq 2 \exp \left[-\min \left(\Re \eta, \Re \eta^{\prime}\right) t\right]\left\{|x|^{\alpha}\left|\xi^{\prime}-\xi\right|^{\alpha}+(\Lambda t)^{\alpha / 2}\left|\left(\eta^{\prime}-\eta\right) / \Lambda\right|^{\alpha / 2}\right\} .
\end{aligned}
$$

Hence from (2.12) the function $g(\cdot, \cdot)$ is in $L^{p}\left(\mathbf{Z}^{d+1}, \mathbf{C}^{d} \otimes \mathbf{C}^{d}\right)$ with $p>(d+2) /$ $(d+2-\alpha)$ and with $\|g(\cdot, \cdot)\|_{p}$ satisfying the inequality

$$
\|g(\cdot, \cdot)\|_{p} \leq C_{p} \Lambda^{1-1 / p}\left[\left|\xi^{\prime}-\xi\right|^{\alpha}+\left|\left(\eta^{\prime}-\eta\right) / \Lambda\right|^{\alpha / 2}\right],
$$

where the constant $C_{p}$ depends only on $d, p$. The Hölder continuity (3.3) for sufficiently small $\alpha>0$ follows from (3.5) and (3.8).

Proof of Theorem 1.2 (Discrete time case). We follow the proof of Theorem 1.1 using the Hölder continuity of the function $q(\cdot, \cdot)$.

For the continuous time case we prove Theorem 1.2 assuming a hypothesis analogous to Hypothesis 3.1. For $1 \leq p \leq \infty$ let $L^{p}\left(\mathbf{Z}^{d} \times \mathbf{R}, \mathbf{C}^{d} \otimes \mathbf{C}^{d}\right)$ be the Banach space of $d \times d$ matrix valued functions $g: \mathbf{Z}^{d} \times \mathbf{R} \rightarrow \mathbf{C}^{d} \otimes \mathbf{C}^{d}$ with norm $\|g\|_{p}$ 
defined by

$$
\begin{aligned}
\|g\|_{p}^{p} & =\sup _{v \in \mathbf{C}^{d}:|v|=1} \sum_{x \in \mathbf{Z}^{d}} \int_{-\infty}^{\infty} d t|g(x, t) v|^{p} \text { if } p<\infty, \\
\|g\|_{\infty} & =\sup _{v \in \mathbf{C}^{d}:|v|=1}\left[\sup _{(x, t) \in \mathbf{Z}^{d} \times \mathbf{R}}|g(x, t) v|\right]
\end{aligned}
$$

where $|g(x, t) v|$ is the Euclidean norm of the vector $g(x, t) v \in \mathbf{C}^{d}$.

Hypothesis 3.2. There exists $p_{0}(\Lambda / \lambda)>1$ depending only on $d, \Lambda / \lambda$ and a constant $C$ such that for $1 \leq p \leq p_{0}(\Lambda / \lambda)$,

$$
\left\|P \sum_{x \in \mathbf{Z}^{d}} \int_{-\infty}^{\infty} d t g(x, t) \mathbf{b}\left(\tau_{x,-t^{*}}\right)\left[v+\partial_{\xi} \Phi\left(\xi, \eta, \tau_{x,-t^{*}}\right) v\right]\right\| \leq C\|g\|_{p}|v|
$$

for all $\xi \in \mathbf{R}^{d}, \eta \in \mathbf{C}$ with $0<\Re \eta<\Lambda$, and $g \in L^{p}\left(\mathbf{Z}^{d} \times \mathbf{R}, \mathbf{C}^{d} \otimes \mathbf{C}^{d}\right), v \in \mathbf{C}^{d}$.

It is easy to see that Hypothesis 3.2 implies the Hölder continuity of the matrix function $q(\cdot, \cdot)$ defined by (2.3),$(2.43)$. We conclude that Theorem 1.2 holds for the continuous time case also.

\section{Fludtuations of averaged Green's functions}

In this section we shall prove Theorem 1.3 under the assumption that the solutions $\Phi(\xi, \eta, \omega)$ of (2.2), (2.43) satisfy stronger versions of Hypothesis 3.1 and 3.2 of $\S 3$. Thus in the discrete time case our hypothesis is:

Hypothesis 4.1. Let $T_{\xi, \eta}$ be the operator 2.10) on the Hilbert space $\mathcal{H}(\Omega)$ and let $T_{\xi, \eta}^{*}$ denote its adjoint. Then for $k \geq 1, p_{2}=p_{3}=\cdots=p_{k}=1$, and $S_{\xi, \eta}=T_{\xi, \eta}$ or $S_{\xi, \eta}=T_{\xi, \eta}^{*}$, there exists $p_{0}(\Lambda / \lambda)>1$ depending only on $d, \Lambda / \lambda$ and a constant $C(k)$ such that

$$
\begin{aligned}
& \left\|\sum_{\left(x_{1}, t_{1}\right), \ldots,\left(x_{k}, t_{k}\right) \in \mathbf{Z}^{d+1}}\left\{\prod_{j=1}^{k} g_{j}\left(x_{j}, t_{j}\right) \tau_{x_{j},-t_{j}} P \mathbf{b}(\cdot)\left[I-P S_{\xi, \eta} \mathbf{b}(\cdot)\right]^{-1}\right\} v\right\| \\
& \leq C(k) \prod_{j=1}^{k}\left\|g_{j}\right\|_{p_{j}}|v| \quad \text { for } g_{j} \in L^{p_{j}}\left(\mathbf{Z}^{d+1}, \mathbf{C}^{d} \otimes \mathbf{C}^{d}\right), j=1, \ldots, k, v \in \mathbf{C}^{d},
\end{aligned}
$$

provided $1 \leq p_{1} \leq p_{0}(\Lambda / \lambda)$ and $\xi \in \mathbf{C}^{d}, \eta \in \mathbf{C}$ satisfy $0<\Re \eta<\Lambda,|\Im \xi| \leq$ $C_{1} \sqrt{\Re \eta / \Lambda}$, with $C_{1}$ depending only on $d, \Lambda / \lambda$.

Remark 5. Note that from (2.13) and Lemma 2.1 we see that the inequality (4.1) holds for $p_{1}=1$. Hence if (4.1) holds for $p_{1}=p_{0}(\Lambda / \lambda)$, by the Riesz convexity theorem [31] it also holds for any $p_{1}$ satisfying $1 \leq p_{1} \leq p_{0}(\Lambda / \lambda)$. 
We define spaces $L^{p}\left([-\pi, \pi]^{d+1} \times \Omega, \mathbf{C}^{d} \otimes \mathbf{C}^{d}\right)$ of $d \times d$ matrix valued functions $g:[-\pi, \pi]^{d+1} \times \Omega \rightarrow \mathbf{C}^{d} \otimes \mathbf{C}^{d}$ with norm $\|g\|_{p}$ defined by

$$
\begin{array}{r}
\|g\|_{p}^{p}=\sup _{v \in \mathbf{C}^{d}:|v|=1} \frac{1}{(2 \pi)^{d+1}} \int_{[-\pi, \pi]^{d+1}}\left\langle|g(\xi, \Im \eta, \cdot) v|^{2}\right\rangle^{p / 2} d[\Im \eta] d \xi \quad \text { if } p<\infty \\
\|g\|_{\infty}=\sup _{v \in \mathbf{C}^{d}:|v|=1}\left[\sup _{(\xi, \Im \eta) \in[-\pi, \pi]^{d+1}}\left\langle|g(\xi, \Im \eta, \cdot) v|^{2}\right\rangle^{1 / 2}\right] .
\end{array}
$$

We consider $\xi \in \mathbf{C}^{d}, \eta \in \mathbf{C}$ with $\xi$ having fixed imaginary part, $\eta$ having fixed positive real part, and satisfying the conditions of Hypothesis 4.1. For $k \geq 1$ we define a multilinear operator $T_{k, \Im \xi, \Re \eta}$ from a sequence $\left[g_{1}, g_{2}, \ldots, g_{k}\right]$ of $k$ functions $g_{j}: \mathbf{Z}^{d+1} \rightarrow \mathbf{C}^{d} \otimes \mathbf{C}^{d}, j=1, \ldots, k$, to periodic functions $T_{k, \Im \xi, \Re \eta}\left[g_{1}, g_{2}, \ldots, g_{k}\right]:$ $[-\pi, \pi]^{d+1} \times \Omega \rightarrow \mathbf{C}^{d} \otimes \mathbf{C}^{d}$ by

$$
=\sum_{\left(x_{1}, t_{1}\right), \ldots,\left(x_{k}, t_{k}\right) \in \mathbf{Z}^{d+1}} \prod_{j=1}^{k} g_{j}\left(x_{j}, t_{j}\right) e^{-i\left(x_{j} . \Re \xi+t \Im \eta\right)} \tau_{x_{j},-t_{j}} P \mathbf{b}(\cdot)\left[I-P T_{\xi, \eta} \mathbf{b}(\cdot)\right]^{-1} \text {. }
$$

Note that the RHS of (4.3) depends on $(\Re \xi$, $\Im \xi)$ and $(\Re \eta, \Im \eta)$ through taking $\xi=$ $\Re \xi+i \Im \xi$ and $\eta=\Re \eta+i \Im \eta$ in the operator $T_{\xi, \eta}$ as well as through the exponential term. We similarly define multilinear operators $\tilde{T}_{k, \Im \xi, \Re \eta}$ by replacing $T_{\xi, \eta}$ in (4.3) with $T_{\xi, \eta}^{*}$. For $p$ satisfying $1 \leq p \leq \infty$ let $p^{\prime}$ be the conjugate of $p$, so $1 / p+1 / p^{\prime}=1$. In [8] the following result was obtained:

Lemma 4.1. Suppose $2 \leq q \leq \infty$ and $p_{1}, \ldots, p_{k}$ with $1 \leq p_{1}, \ldots, p_{k} \leq 2$ satisfy the identity

$$
\frac{1}{p_{1}^{\prime}}+\frac{1}{p_{2}^{\prime}}+\cdots+\frac{1}{p_{k}^{\prime}}=\frac{1}{q}
$$

and for $j=1, \ldots, k$, the functions $g_{j} \in L^{p_{j}}\left(\mathbf{Z}^{d+1}, \mathbf{C}^{d} \otimes \mathbf{C}^{d}\right)$. Then there exist positive constants $C_{1}, C_{2}$ depending only on $d, \Lambda / \lambda$ such that if $0<\Re \eta<$ $\Lambda,|\Im \xi|<C_{1} \sqrt{\Re \eta / \Lambda}$, the function $S_{k, \Im \xi, \Re \eta}\left[g_{1}, g_{2}, \ldots, g_{k}\right]=T_{k, \Im \xi, \Re \eta}\left[g_{1}, g_{2}, \ldots, g_{k}\right]$ or $S_{k, \Im \xi, \Re \eta}\left[g_{1}, g_{2}, \ldots, g_{k}\right]=\tilde{T}_{k, \Im \xi, \Re \eta}\left[g_{1}, g_{2}, \ldots, g_{k}\right]$ is in $L^{q}\left([-\pi, \pi]^{d+1} \times \Omega, \mathbf{C}^{d} \otimes \mathbf{C}^{d}\right)$ and

$$
\left\|S_{k, \Im \xi, \Re \eta}\left[g_{1}, g_{2}, \ldots, g_{k}\right]\right\|_{q} \leq C_{2}^{k} \prod_{j=1}^{k}\left\|g_{j}\right\|_{p_{j}} .
$$

If we assume Hypothesis 4.1 we can improve Lemma 4.1 as follows:

Lemma 4.2. Suppose Hypothesis 4.1 holds with $p_{0}(\Lambda / \lambda) \leq 2$, and $q, p_{1}, \ldots, p_{k}$ with $2 \leq q \leq \infty, 1 \leq p_{1}, \ldots, p_{k} \leq 2$ satisfy the inequality

$$
\frac{1}{q} \leq \frac{1}{p_{1}^{\prime}}+\frac{1}{p_{2}^{\prime}}+\cdots+\frac{1}{p_{k}^{\prime}} \leq \frac{1}{q}+\left[1-\frac{1}{p_{0}(\Lambda / \lambda)}\right]\left[1-\frac{2}{q}\right] .
$$


Then there exists a positive constant $C_{1}$ depending only on $d$ and $\Lambda / \lambda$ such that if $0<\Re \eta<\Lambda$ and $|\Im \xi|<C_{1} \sqrt{\Re \eta / \Lambda}$, the function $S_{k, \Im \xi, \Re \eta}\left[g_{1}, g_{2}, \ldots, g_{k}\right]=$ $T_{k, \Im \xi, \Re \eta}\left[g_{1}, g_{2}, \ldots, g_{k}\right]$ or $S_{k, \Im \xi, \Re \eta}\left[g_{1}, g_{2}, \ldots, g_{k}\right]=\tilde{T}_{k, \Im \xi, \Re \eta}\left[g_{1}, g_{2}, \ldots, g_{k}\right]$ is in $L^{q}\left([-\pi, \pi]^{d+1} \times \Omega, \mathbf{C}^{d} \otimes \mathbf{C}^{d}\right)$ and

$$
\left\|S_{k, \Im \xi, \Re \eta}\left[g_{1}, g_{2}, \ldots, g_{k}\right]\right\|_{q} \leq C(k) \prod_{j=1}^{k}\left\|g_{j}\right\|_{p_{j}},
$$

for some constant $C(k)$.

Proof. We assume first that $p_{2}=p_{3}=\cdots=p_{k}=1$, in which case Hypothesis 4.1 and Lemma 4.1 imply respectively that (4.7) holds for $1 / p_{1}^{\prime} \leq 1-1 / p_{0}(\Lambda / \lambda), q=$ $\infty$, and for $p_{1}=q=2$. The Riesz convexity theorem then implies that (4.7) holds if $p_{1}^{\prime}, q$ satisfy (4.6) with $p_{2}=p_{3}=\cdots=p_{k}=1$. Next assume for induction that we have proved (4.7) in the case when (4.6) holds with $p_{r+1}=$ $p_{r+2}=\cdots=p_{k}=1$ for some $r \geq 1$. Hence (4.7) holds for $1 / p_{1}^{\prime}+\cdots+1 / p_{r}^{\prime} \leq$ $1-1 / p_{0}(\Lambda / \lambda), p_{r+1}=1, q=\infty$, where the functions $g_{r+1}, \ldots, g_{k}$ are fixed with $p_{r+2}=p_{r+3}=\cdots=p_{k}=1$. From Lemma 4.1 we see that (4.7) also holds for $1 / p_{1}^{\prime}+\cdots+1 / p_{r+1}^{\prime}=1 / 2, q=2$, with the same functions $g_{r+1}, \ldots, g_{k}$. Now we fix the functions $g_{1}, \ldots, g_{r}, g_{r+2}, \ldots, g_{k}$ with $p_{r+1}=p_{r+2}=\cdots=p_{k}=1$ and $1 / p_{1}^{\prime}+\cdots+1 / p_{r}^{\prime} \leq 1-1 / p_{0}(\Lambda / \lambda) \leq 1 / 2$. Applying the Riesz convexity theorem to the functions $g_{r+1}$, we conclude that (4.7) holds if $p_{1}, \ldots, p_{r+1}$ satisfies (4.6) with $p_{r+2}=p_{r+3}=\cdots=p_{k}=1$.

For $1 \leq p<\infty$ let $L_{w}^{p}\left([-\pi, \pi]^{d+1}\right)$ be the space of functions $g:[-\pi, \pi]^{d+1} \rightarrow \mathbf{C}$ which are weakly $p$ integrable. The norm $\|g\|_{p, w}$ of $g$ is defined to be the minimum number satisfying the inequality

$(2 \pi)^{-(d+1)} \operatorname{meas}\left\{(\xi, \Im \eta) \in[-\pi, \pi]^{d+1}:|g(\xi, \Im \eta)|>z\right\} \leq\|g\|_{p, w}^{p} / z^{p} \quad$ for all $z>0$.

Proposition 4.1. Assume Hypothesis 4.1 holds, $4 d \Lambda \leq 1$ and $m$ is a positive integer. Then there exist positive constants $C_{1}$ and $\alpha \leq 1$ depending only on $d$ and $\Lambda / \lambda$ such that

$$
\begin{aligned}
& \left\|q\left(\xi^{\prime}, \eta^{\prime}\right)-q(\xi, \eta)\right\| \leq C \Lambda\left[\left|\xi^{\prime}-\xi\right|^{\alpha}+\left|\left(\eta^{\prime}-\eta\right) / \Lambda\right|^{\alpha / 2}\right], \\
& \quad \text { for } 0<\Re \eta, \Re \eta^{\prime} \leq \Lambda, \quad \xi^{\prime}, \xi \in \mathbf{C}^{d} \text { with }|\Im \xi|+\left|\Im \xi^{\prime}\right| \leq C_{1} \sqrt{\Re \eta / \Lambda},
\end{aligned}
$$

where $C$ is a constant.

If $\xi \in \mathbf{C}^{d}, \eta \in \mathbf{C}$ with fixed $\Im \xi \in \mathbf{R}^{d}, \Re \eta>0$ satisfying $0<\Re \eta<\Lambda,|\Im \xi|<$ $C_{1} \sqrt{\Re \eta / \Lambda}$, and $m<1+d / 2$, then the function

$$
(\Re \xi, \Im \eta) \rightarrow \frac{\partial^{m} q_{r, r^{\prime}}(\xi, \eta)}{\partial \eta^{m}}, \quad(\Re \xi, \Im \eta) \in[-\pi, \pi]^{d+1},
$$

is in the space $L_{w}^{p}\left([-\pi, \pi]^{d+1}\right)$ with $p=(1+d / 2) /(m-\alpha / 2)$ and its norm is bounded by $C \Lambda^{1-m+1 / p}$ for some constant $C$.

If $m$ is the largest integer strictly less than $1+d / 2$ and $0 \leq \delta<1+d / 2-m$, then for any $\rho \in \mathbf{R}$ satisfying $|\rho| \leq 1$, the function

$$
(\Re \xi, \Im \eta) \rightarrow \frac{\partial^{m}}{\partial \eta^{m}}\left[q_{r, r^{\prime}}(\xi, \eta+i \rho)-q_{r, r^{\prime}}(\xi, \eta)\right] /|\rho|^{\delta}
$$


is in the space $L_{w}^{p}\left([-\pi, \pi]^{d+1}\right)$ with $p=(1+d / 2) /(m+\delta-\alpha / 2)$ and its norm is bounded by $C_{p} \Lambda^{1-m-\delta+1 / p}$, where the constant $C_{p}$ can diverge as $p \rightarrow 1$.

Proof. The Hölder continuity (4.9) of the function $q(\cdot, \cdot)$ has already been proven in Lemma 3.1. We first prove that the derivative (4.10) with $m=1$ is in $L_{w}^{p}\left([-\pi, \pi]^{d+1}\right)$ with $p=(1+d / 2) /(1-\alpha / 2)$ for some $\alpha>0$ depending only on $d, \Lambda / \lambda$. Observe from (2.20) and (2.21) that

$$
\left(\frac{\partial}{\partial \eta}\right) q(\xi, \eta)=-\Lambda\left\langle\mathbf{b}(\cdot)\left[I-P T_{\xi, \eta} \mathbf{b}(\cdot)\right]^{-1}\left\{\frac{\partial}{\partial \eta} T_{\xi, \eta}\right\} P \mathbf{b}(\cdot)\left[I-P T_{\xi, \eta} \mathbf{b}(\cdot)\right]^{-1}\right\rangle .
$$

Denoting by $[\cdot, \cdot]$ the inner product for $\mathcal{H}(\Omega)$, we therefore have for $v_{1}, v_{2} \in \mathbf{C}^{d}$ that (4.13)

$$
\left(\frac{\partial}{\partial \eta}\right) v_{1}^{*} q(\xi, \eta) v_{2}=-\Lambda\left[\tilde{T}_{1, \Im \xi, \Re \eta} g(\Re \xi, \Im \eta, \cdot) v_{1}, T_{1, \Im \xi, \Re \eta} h(\Re \xi, \Im \eta, \cdot) v_{2}\right]
$$

for certain $d \times d$ matrix valued functions $g(x, t), h(x, t), x \in \mathbf{Z}^{d}, t \in \mathbf{Z}$. The functions $g(\cdot, \cdot), h(\cdot, \cdot)$ are determined from their Fourier transforms (2.15) by the formula

$$
\hat{g}(\zeta, \theta)^{*} \hat{h}(\zeta, \theta)=-\frac{\Lambda e(i \Im \xi-\zeta) e(-i \Im \xi-\zeta)^{*}}{\left[e^{\Re \eta-i \theta}-1+\Lambda e(-i \Im \xi-\zeta)^{*} e(i \Im \xi-\zeta)\right]^{2}},
$$

which follows from (2.19). We take $\hat{h}(\cdot, \cdot)$ to be given by the formula

$$
\hat{h}(\zeta, \theta)=\left(\frac{\Lambda}{d}\right)^{1 / 2} \frac{\mathbf{1}_{d} e(-i \Im \xi-\zeta)^{*}}{\left[e^{\Re \eta-i \theta}-1+\Lambda e(-i \Im \xi-\zeta)^{*} e(i \Im \xi-\zeta)\right]},
$$

where $\mathbf{1}_{d}$ is the $d$ dimensional column vector with all entries equal to 1 . From (2.11) and (4.15) it follows that

$$
h(x, t)=\left(\frac{\Lambda}{d}\right)^{1 / 2} \mathbf{1}_{d}\left\{\nabla G_{\Lambda}(x, t-1)\right\}^{*} e^{x \cdot \Im \xi-t \Re \eta} \text { if } t \geq 1, \quad h(x, t)=0 \text { otherwise. }
$$

Assuming $0<\Re \eta<\Lambda,|\Im \xi| \leq C \sqrt{\Re \eta / \Lambda}$, for sufficiently small positive constant $C$ depending only on $d$, it follows from (2.12), (4.16) that $h(\cdot, \cdot)$ is in $L_{w}^{p}\left(\mathbf{Z}^{d+1}\right)$ with $p=(d+2) /(d+1)$ and $\|h\|_{p, w} \leq C \Lambda^{1 / 2-1 / p}$ for a constant $C$ depending only on $d$.

Observe now that by the Hunt interpolation theorem [29] the inequality (4.7) also holds for the operator $T_{1, \Im \xi, \Re \eta}$ as a mapping from $L_{w}^{p_{1}}\left(\mathbf{Z}^{d+1}\right)$ to $L_{w}^{q}\left([-\pi, \pi]^{d+1} \times\right.$ $\left.\Omega, \mathbf{C}^{d} \otimes \mathbf{C}^{d}\right)$. Hence $T_{1, \Im \xi, \Re \eta} h$ is in $L_{w}^{q}\left([-\pi, \pi]^{d+1} \times \Omega, \mathbf{C}^{d} \otimes \mathbf{C}^{d}\right)$ provided $q$ satisfies the inequality in (4.6) with $p_{1}=(d+2) /(d+1)$. Evidently we can choose $q$ so that $q / 2>1+d / 2$. Since we can make an exactly similar argument for the function $g(x, t)$ and $\tilde{T}_{1, \Im \xi, \Re \eta} g$, we conclude from (4.13) that $\partial q_{r, r^{\prime}}(\xi, \eta) / \partial \eta$ is in the space $L_{w}^{q / 2}\left([-\pi, \pi]^{d+1}\right)$ with norm bounded by $\Lambda^{2-2 / p}$ times a constant. We have proved for $m=1$ that the derivative (4.10) is in the appropriate weak $L^{p}$ space. We proceed similarly to estimate the higher derivatives (4.10) and the fractional derivative (4.11).

Remark 6 . Proposition 4.1 with $\alpha=0$ was proven in 8 . In that case the constant $C$ in the statement of the proposition depends only on $d, \Lambda / \lambda$.

Proposition 4.1 enables us to compare the averaged Green's function $G_{\mathbf{a}}(x, t)$, $x \in \mathbf{Z}^{d}, t \in \mathbf{Z}^{+}$for (1.2), (1.3) to the lattice Green's function $G_{\mathbf{a}_{\text {hom }}}^{\text {lattice }}(x, t), x \in \mathbf{Z}^{d}$, 
$t \in \mathbf{Z}^{+}$defined by

$$
G_{\mathbf{a}_{\text {hom }}}^{\text {lattice }}(x, t)=\frac{1}{(2 \pi)^{d+1}} \int_{[-\pi, \pi]^{d+1}} \frac{e^{-i \xi \cdot x+\eta(t+1)}}{e^{\eta}-1+e(\xi)^{*} q(0,0) e(\xi)} d[\Im \eta] d \xi .
$$

Theorem 4.1. Assume Hypothesis 4.1 holds and $4 d \Lambda \leq 1$. Then there exist positive constants $\alpha, \gamma$, with $\alpha \leq 1$, depending only on $d, \Lambda / \lambda$ and a constant $C$ such that for $x \in \mathbf{Z}^{d}, t \in \mathbf{Z}, t \geq 0$,

$$
\left|G_{\mathbf{a}}(x, t)-G_{\mathbf{a}_{\text {hom }}}^{\text {lattice }}(x, t)\right| \leq \frac{C}{[\Lambda t+1]^{(d+\alpha) / 2}} \exp \left[-\gamma \min \left\{|x|, \frac{|x|^{2}}{\Lambda t+1}\right\}\right],
$$

$$
\left|\nabla G_{\mathbf{a}}(x, t)-\nabla G_{\mathbf{a}_{\text {hom }}}^{\text {lattice }}(x, t)\right| \leq \frac{C}{[\Lambda t+1]^{(d+1+\alpha) / 2}} \exp \left[-\gamma \min \left\{|x|, \frac{|x|^{2}}{\Lambda t+1}\right\}\right] .
$$

If $\delta$ satisfies $0<\delta \leq 1$, then there exist $\alpha, \gamma>0$ depending only on $d, \Lambda / \lambda, \delta$ and $a$ constant $C_{\delta}$ such that the following inequality holds:

$$
\begin{aligned}
& \left|\left[\nabla G_{\mathbf{a}}\left(x^{\prime}, t\right)-\nabla G_{\mathbf{a} \text { hom }}^{\text {lattice }}\left(x^{\prime}, t\right)\right]-\left[\nabla G_{\mathbf{a}}(x, t)-\nabla G_{\mathbf{a}_{\text {hom }}}^{\text {lattice }}(x, t)\right]\right| \\
& \leq\left|x^{\prime}-x\right|^{1-\delta} \frac{C_{\delta}}{[\Lambda t+1]^{(d+2-\delta+\alpha) / 2}} \exp \left[-\gamma \min \left\{|x|, \frac{|x|^{2}}{\Lambda t+1}\right\}\right], \\
& x^{\prime}, x \in \mathbf{Z}^{d}, 1 / 2 \leq\left(\left|x^{\prime}\right|+1\right) /(|x|+1) \leq 2 .
\end{aligned}
$$

The constant $\alpha$ in (4.20) must satisfy $\alpha<\delta$.

Proof. From (2.8), (4.17) and Corollary 2.1 there is a constant $C$ depending only on $\Lambda / \lambda$ such that for $a \in \mathbf{R}^{d}$ with $|a| \leq 1$,

$$
\begin{aligned}
& G_{\mathbf{a}}(x, t)-G_{\mathbf{a}_{\text {hom }}}^{\text {lattice }}(x, t) \\
& \quad=\frac{\exp \left[a \cdot x / C+\Lambda|a|^{2}(t+1)\right]}{(2 \pi)^{d+1}} \int_{[-\pi, \pi]^{d+1}} e^{-i \xi \cdot x+i \Im \eta(t+1)} f_{a}(\xi, \Im \eta) d \xi d[\Im \eta],
\end{aligned}
$$

where the function $f_{a}(\xi, \Im \eta)$ is given by the formula

$$
\begin{aligned}
& f_{a}(\xi, \Im \eta)=\frac{e(\xi-i a / C)^{*}\left\{q(0,0)-q\left(\xi+i a / C, \Lambda|a|^{2}+i \Im \eta\right)\right\} e(\xi+i a / C)}{\left[\exp \left[\Lambda|a|^{2}+i \Im \eta\right]-1+e(\xi-i a / C)^{*} q(0,0) e(\xi+i a / C)\right]} \\
& \times \frac{1}{\left[\exp \left[\Lambda|a|^{2}+i \Im \eta\right]-1+e(\xi-i a / C)^{*} q\left(\xi+i a / C, \Lambda|a|^{2}+i \Im \eta\right) e(\xi+i a / C)\right]} .
\end{aligned}
$$

The exponential decay in the inequalities (4.18)-(4.20) is obtained by choosing $a$ in (4.21) to be given by

(4.23) $a=-x /(C+1)(\Lambda t+1)$ if $|x| \leq \Lambda t+1, \quad a=-x /(C+1)|x|$ if $|x| \geq \Lambda t+1$.

It follows from (2.29), Proposition 4.1 and Corollary 2.1 that there is a positive constant $C_{1}$ such that the function in (4.22) is bounded by

$$
\left|f_{a}(\xi, \Im \eta)\right| \leq \frac{C_{1}\left[|e(\xi)|^{2}+|a|^{2}\right]}{\Lambda\left[|\Im \eta| / \Lambda+|e(\xi)|^{2}+|a|^{2}\right]^{2-\alpha / 2}}, \quad \text { for }(\xi, \Im \eta) \in[-\pi, \pi]^{d+1} \text {. }
$$

To complete the proof of the theorem we need to obtain the polynomial decay in $[\Lambda t+1]$ in (4.18)-(4.20), whence we may assume that $\Lambda t \geq 1$. We divide the torus $[-\pi, \pi]^{d+1}$ into various regions, the first of which is

$$
E_{0,0}=\left\{(\xi, \Im \eta) \in[-\pi, \pi]^{d+1}: \Lambda t|e(\xi)|^{2} \leq 1,|\Im \eta| \leq 1 / t\right\} .
$$


It follows then from (4.24) that there is a constant $C_{2}$ such that

$$
\int_{E_{0,0}}\left|f_{a}(\xi, \Im \eta)\right| d \xi d[\Im \eta] \leq C_{2} /[\Lambda t+1]^{(d+\alpha) / 2} .
$$

Next we consider for $k=1,2, \ldots$, regions

$$
E_{0, k}=\left\{(\xi, \Im \eta) \in[-\pi, \pi]^{d+1}: \Lambda t|e(\xi)|^{2} \leq 1, \quad 2^{k-1} / t<|\Im \eta| \leq 2^{k} / t\right\}
$$

From (4.24) we see that if $|a| \leq 2 / \Lambda t$ there is a constant $C_{3}$ such that $(4.28)$

$$
\left|\int_{E_{0, k}} e^{-i \xi \cdot x+i \Im \eta(t+1)} f_{a}(\xi, \Im \eta) d \xi d[\Im \eta]\right| \leq C_{3} 2^{-k(1-\alpha / 2)} /[\Lambda t+1]^{(d+\alpha) / 2} .
$$

In general $a=O(1)$, so we need to take advantage of the oscillatory nature of the integral in (4.28). Let $\rho=\pi /(t+1)$ so that $e^{i \rho(t+1)}=-1$, and $E_{0, k}^{\rho}=\{(\xi, \Im \eta)$ : $\left.(\xi, \Im \eta+\rho) \in E_{0, k}\right\}$. Then the LHS of (4.28) is bounded by

$$
\begin{aligned}
& \frac{1}{2} \int_{E_{0, k} \cap E_{0, k}^{\rho}}\left|f_{a}(\xi, \Im \eta)-f_{a}(\xi, \Im \eta+\rho)\right| d \xi d[\Im \eta] \\
+ & \frac{1}{2} \int_{E_{0, k}-E_{0, k}^{\rho}}\left|f_{a}(\xi, \Im \eta)\right| d \xi d[\Im \eta]+\frac{1}{2} \int_{E_{0, k}^{\rho}-E_{0, k}}\left|f_{a}(\xi, \Im \eta+\rho)\right| d \xi d[\Im \eta] .
\end{aligned}
$$

It follows again from (4.24) that the last two integrals on the RHS of (4.29) are bounded by the RHS of (4.28). In order to bound the first integral we observe from the Hölder continuity (4.9) of the function $q(\cdot, \cdot)$ that there are constants $C_{4}, C_{5}$ and

$$
\begin{aligned}
\mid f_{a}(\xi, \Im \eta) & -f_{a}(\xi, \Im \eta+\rho) \mid \leq \frac{C_{4}\left[|e(\xi)|^{2}+|a|^{2}\right](\rho / \Lambda)^{\alpha / 2}}{\Lambda\left[|\Im \eta| / \Lambda+|e(\xi)|^{2}+|a|^{2}\right]^{2}} \\
& +\frac{C_{5}\left[|e(\xi)|^{2}+|a|^{2}\right](\rho / \Lambda)}{\Lambda\left[|\Im \eta| / \Lambda+|e(\xi)|^{2}+|a|^{2}\right]^{3-\alpha / 2}}, \quad \text { for }(\xi, \Im \eta) \in E_{0, k} \cap E_{0 . k}^{\rho}
\end{aligned}
$$

Since we are assuming $|a| \geq 2 / \Lambda t$ it follows from (4.30) that

$$
\sum_{k \geq 1} \int_{E_{0, k} \cap E_{0, k}^{\rho}}\left|f_{a}(\xi, \Im \eta)-f_{a}(\xi, \Im \eta+\rho)\right| d \xi d[\Im \eta] \leq C_{6} /[\Lambda t+1]^{(d+\alpha) / 2}
$$

for some constant $C_{6}$. We therefore conclude from (4.26)-(4.31) that there is a constant $C_{7}$ and

$$
\sum_{k \geq 0}\left|\int_{E_{0, k}} e^{-i \xi \cdot x+i \Im \eta(t+1)} f_{a}(\xi, \Im \eta) d \xi d[\Im \eta]\right| \leq C_{7} /[\Lambda t+1]^{(d+\alpha) / 2} .
$$

The inequality (4.32) can also be derived by using the fact from Theorem 3.1 that the derivative $\partial q\left(\xi+i a / C, \Lambda|a|^{2}+i \Im \eta\right) / \partial[\Im \eta]$ is in the space $L_{w}^{p}\left([-\pi, \pi]^{d+1}\right)$ with $p=(1+d / 2) /(1-\alpha / 2)$. Thus we observe that

$$
\begin{array}{r}
\left|\int_{E_{0, k}} e^{-i \xi \cdot x+i \Im \eta(t+1)} f_{a}(\xi, \Im \eta) d \xi d[\Im \eta]\right| \leq \frac{1}{t+1} \int_{\partial E_{0, k}}\left|f_{a}(\xi, \Im \eta)\right| d \xi \\
+\frac{1}{t+1} \int_{E_{0, k}}\left|\frac{\partial f_{a}(\xi, \Im \eta)}{\partial[\Im \eta]}\right| d \xi d[\Im \eta]
\end{array}
$$


where $\partial E_{0, k}$ is the union of sets $\left\{(\xi, \Im \eta): \Lambda t|e(\xi)|^{2} \leq 1\right.$, $\Im \eta=$ constant $\}$ with the constant given by $\pm 2^{k} / t$ or $\pm 2^{k-1} / t$. It follows from (4.24) that the first integral on the RHS of (4.33) is bounded by the RHS of (4.28). To bound the second integral we use the inequality

$$
\begin{aligned}
\left|\frac{\partial f_{a}(\xi, \Im \eta)}{\partial[\Im \eta]}\right| \leq \frac{C_{8}\left[|e(\xi)|^{2}+|a|^{2}\right]}{\Lambda^{2}\left[|\Im \eta| / \Lambda+|e(\xi)|^{2}+|a|^{2}\right]^{3-\alpha / 2}} \\
+\frac{C_{9}\left[|e(\xi)|^{2}+|a|^{2}\right]}{\Lambda^{2}\left[|\Im \eta| / \Lambda+|e(\xi)|^{2}+|a|^{2}\right]^{2}}\left|\frac{\partial q\left(\xi+i a / C, \Lambda|a|^{2}+i \Im \eta\right)}{\partial[\Im \eta]}\right|,
\end{aligned}
$$

where $C_{8}, C_{9}$ are constants. We can bound the integral of the first term on the RHS of (4.34) just as we did with the second term on the RHS of (4.30). To bound the integral of the second term we use the well known fact that if $f \in L_{w}^{p}\left([-\pi, \pi]^{d+1}\right)$ with $1<p<\infty$, then for any measurable set $F$, one has

$$
\int_{F}|f| \leq C_{p}\|f\|_{p, w} m(F)^{1-1 / p},
$$

where the constant $C_{p}$ depends only on $p$. Taking $p=(1+d / 2) /(1-\alpha / 2)$ we conclude from Proposition 4.1 that $1 /(t+1)$ times the integral over $E_{0, k}$ of the second term on the RHS of (4.34) is bounded by

$$
\frac{C_{10}\left[1 / \Lambda t+|a|^{2}\right]}{\Lambda t\left[2^{k} / \Lambda t+|a|^{2}\right]^{2}} \frac{2^{k(1-1 / p)}}{[\Lambda t+1]^{(d+\alpha) / 2}}
$$

for some constant $C_{10}$. Summing (4.36) over $k \geq 1$ we obtain the inequality (4.32) again.

For $r \geq 1, k \geq 0$, let $E_{r, k}$ be defined by

$$
\begin{gathered}
E_{r, k}=\left\{(\xi, \Im \eta) \in[-\pi, \pi]^{d+1}: 2^{r-1}<\Lambda t|e(\xi)|^{2} \leq 2^{r},\right. \\
\\
\left.2^{k-1} / t<|\Im \eta| \leq 2^{k} / t\right\}, k \geq 1, \\
E_{r, 0}=\left\{(\xi, \Im \eta) \in[-\pi, \pi]^{d+1}: 2^{r-1}<\Lambda t|e(\xi)|^{2} \leq 2^{r}, \quad|\Im \eta| \leq 1 / t\right\} .
\end{gathered}
$$

Then we have that

$$
\begin{aligned}
\sum_{k=0}^{\infty} \int_{E_{r, k}} e^{-i \xi \cdot x+i \Im \eta(t+1)} f_{a}(\xi, \Im \eta) d \xi d[\Im \eta] & \\
= & \frac{i^{m}}{(t+1)^{m}} \sum_{k=0}^{\infty} \int_{E_{r, k}} e^{-i \xi \cdot x+i \Im \eta(t+1)} \frac{\partial^{m} f_{a}(\xi, \Im \eta)}{\partial[\Im \eta]^{m}} d \xi d[\Im \eta]
\end{aligned}
$$

Just as in (4.34) we see from Proposition 4.1 that

$$
\frac{\partial^{m} f_{a}(\xi, \Im \eta)}{\partial[\Im \eta]^{m}}=\frac{|e(\xi)|^{2}+|a|^{2}}{\Lambda^{2}\left[|\Im \eta| / \Lambda+|e(\xi)|^{2}+|a|^{2}\right]^{2}} g_{a, m}(\xi, \Im \eta)
$$

where for $m<1+d / 2$ the function $g_{a, m}(\cdot, \cdot)$ is in $L_{w}^{p}\left([-\pi, \pi]^{d+1}\right)$ with $p=$ $(1+d / 2) /(m-\alpha / 2)$. Thus there is a constant $C_{11}$ such that

$$
\int_{F}\left|g_{a, m}(\xi, \Im \eta)\right| d \xi d[\Im \eta] \leq C_{11} \Lambda^{1-m+1 / p} m(F)^{1-1 / p}, \quad F \subset[-\pi, \pi]^{d+1}
$$

It follows from (4.39), 4.40) that

$$
\frac{1}{(t+1)^{m}} \int_{E_{r, k}}\left|\frac{\partial^{m} f_{a}(\xi, \Im \eta)}{\partial[\Im \eta]^{m}}\right| d \xi d[\Im \eta] \leq \frac{C_{12}}{[\Lambda t+1]^{(d+\alpha) / 2}} \frac{2^{(r d / 2+k)(1-1 / p)}}{2^{r}+2^{k}}
$$


for some constant $C_{12}$. Observe that

$$
\sum_{k=0}^{\infty} \sum_{r=1}^{\infty} \frac{2^{(r d / 2+k)(1-1 / p)}}{2^{r}+2^{k}}<\infty
$$

provided $m$ satisfies the inequality $m>(d+\alpha) / 2$. If $d$ is odd, then there is an integer $m$ satisfying $d / 2<m<1+d / 2$, whence (4.32), (4.38), and (4.41) imply that (4.18) holds for some $\alpha>0$.

In the case when $d$ is even we note from (4.38) that

$$
\sum_{k=0}^{\infty} \int_{E_{r, k}} e^{-i \xi \cdot x+i \Im \eta(t+1)} f_{a}(\xi, \Im \eta) d \xi d[\Im \eta]
$$

$$
=\frac{i^{m}}{2(t+1)^{m}} \sum_{k=0}^{\infty} \int_{E_{r, k}} e^{-i \xi \cdot x+i \Im \eta(t+1)}\left[\frac{\partial^{m} f_{a}(\xi, \Im \eta)}{\partial[\Im \eta]^{m}}-\frac{\partial^{m} f_{a}(\xi, \Im \eta+\rho)}{\partial[\Im \eta]^{m}}\right] d \xi d[\Im \eta],
$$

where $m$ is the largest integer satisfying $m<1+d / 2$ and $\rho=\pi /(t+1)$. Similarly to (4.39) we have that

$$
\begin{aligned}
\frac{1}{|\rho|^{\delta}} & {\left[\frac{\partial^{m} f_{a}(\xi, \Im \eta)}{\partial[\Im \eta]^{m}}-\frac{\partial^{m} f_{a}(\xi, \Im \eta+\rho)}{\partial[\Im \eta]^{m}}\right] } \\
= & \frac{|e(\xi)|^{2}+|a|^{2}}{\Lambda^{2}\left[|\Im \eta| / \Lambda+|e(\xi)|^{2}+|a|^{2}\right]^{2}} g_{a, \delta}(\xi, \Im \eta),
\end{aligned}
$$

where for $0 \leq \delta<1+d / 2-m$ the function $g_{a, \delta}(\cdot, \cdot)$ satisfies an inequality (4.35) with $p=(1+d / 2) /(m+\delta-\alpha / 2)$. Hence as in (4.41) we conclude that

$$
\begin{aligned}
& \frac{1}{(t+1)^{m}} \int_{E_{r, k}}\left|\frac{\partial^{m} f_{a}(\xi, \Im \eta)}{\partial[\Im \eta]^{m}}-\frac{\partial^{m} f_{a}(\xi, \Im \eta+\rho)}{\partial[\Im \eta]^{m}}\right| d \xi d[\Im \eta] \\
& \quad \leq \frac{C_{13}}{[\Lambda t+1]^{(d+\alpha) / 2}} \frac{2^{(r d / 2+k)(1-1 / p)}}{2^{r}+2^{k}},
\end{aligned}
$$

where $C_{13}$ also depends on $\delta$ as well as on $\Lambda, d, \Lambda / \lambda$. Now 4.18) for some $\alpha>0$ follows from (4.32), (4.43), and (4.45) by choosing $\delta$ in (4.45) so that $0<\delta<1$.

In order to prove (4.19) we follow the previous argument, replacing the function $f_{a}(\xi, \Im \eta)$ by the function $e(\xi) f_{a}(\xi, \Im \eta)$. To prove (4.20) we use the inequality

$$
\left|e^{i \xi \cdot\left(x-x^{\prime}\right)}-1\right| \leq 10\left|x-x^{\prime}\right|^{1-\delta}|e(\xi)|^{1-\delta}
$$

and replace the function $f_{a}(\xi, \Im \eta)$ by the function $|e(\xi)|^{2-\delta} f_{a}(\xi, \Im \eta)$ in the argument to prove (4.18).

Remark 7. In the case when $\alpha=0$ the constant $C$ in (4.18), (4.19) depends only on $d, \Lambda / \lambda$. For $\alpha>0$ the constant $C$ also depends on the constant in the inequality (4.1) of Hypothesis 4.1.

The inequalities (1.19), (1.20) of Theorem 1.3 are a consequence now of Theorem 4.1 and the following result which compares the lattice Green's function $G_{\mathbf{a}_{\text {hom }}}^{\text {lattice }}(x, t)$ to the Green's function $G_{\mathbf{a}_{\text {hom }}}(x, t)$ for the PDE (1.6):

Lemma 4.3. Assuming $4 d \Lambda \leq 1$, there exist positive constants $\gamma, C$ depending only on $d, \Lambda / \lambda$ such that for $x \in \mathbf{Z}^{\bar{d}}, t \in \mathbf{Z}$ with $\Lambda t \geq 1$,

$$
\left|G_{\mathbf{a}_{\mathrm{hom}}}(x, t)-G_{\mathbf{a}_{\mathrm{hom}}}^{\text {lattice }}(x, t)\right| \leq \frac{C}{[\Lambda t+1]^{(d+1) / 2}} \exp \left[-\gamma \min \left\{|x|, \frac{|x|^{2}}{\Lambda t+1}\right\}\right],
$$




$$
\left|\nabla G_{\mathbf{a}_{\mathrm{hom}}}(x, t)-\nabla G_{\mathbf{a}_{\mathrm{hom}}}^{\text {lattice }}(x, t)\right| \leq \frac{C}{[\Lambda t+1]^{(d+2) / 2}} \exp \left[-\gamma \min \left\{|x|, \frac{|x|^{2}}{\Lambda t+1}\right\}\right],
$$

$$
\left|\nabla \nabla G_{\mathbf{a}_{\text {hom }}}(x, t)-\nabla \nabla G_{\mathbf{a}_{\text {hom }}}^{\text {lattice }}(x, t)\right| \leq \frac{C}{[\Lambda t+1]^{(d+3) / 2}} \exp \left[-\gamma \min \left\{|x|, \frac{|x|^{2}}{\Lambda t+1}\right\}\right] \text {. }
$$

Proof. Taking $\mathbf{a}_{\text {hom }}=q(0,0)$ in (1.6), we see from (4.17) that $G_{\mathbf{a}_{\text {hom }}}^{\text {lattice }(\cdot, \cdot)}$ is the Green's function for the discrete parabolic equation corresponding to (1.6),

$$
u(x, t+1, \omega)-u(x, t, \omega)=-\nabla^{*} \mathbf{a}_{\mathrm{hom}} \nabla u(x, t, \omega), \quad x \in \mathbf{Z}^{d}, t=0,1,2, \ldots
$$

To prove the theorem we follow a standard method of numerical analysis for estimating error between the solution of a continuous problem and its approximating discrete problems. The method is to regard the solution of the continuous problem as an approximate solution to the discrete problem. An alternative approach based on comparison of the Fourier representation (4.17) of the lattice Green's function $G_{\mathbf{a}_{\text {hom }}}^{\text {lattice }}(\cdot, \cdot)$ to the Fourier representation of the continuous Green's function $G_{\mathbf{a}_{\text {hom }}}(\cdot, \cdot)$ is pursued in 22 for the case of elliptic equations.

Let $f: \mathbf{R}^{d} \rightarrow \mathbf{R}$ be a non-negative $C^{\infty}$ function with support contained in the ball $\left\{x \in \mathbf{R}^{d}:|x|<1\right\}$ and let $u(x, t)=u_{\text {hom }}(x, t)$ be the solution to the initial value problem (1.6), (1.7). With $\nabla_{x}, \nabla_{x}^{*}$ denoting the discrete operators (1.5), we have that

$$
\begin{aligned}
& u(x+z, t+1)-u(x+z, t)+\nabla_{x}^{*} \mathbf{a}_{\mathrm{hom}} \nabla_{x} u(x+z, t) \\
& =u(x+z, t+1)-u(x+z, t) \\
& \quad+\text { Trace }\left[\mathbf{a}_{\mathrm{hom}} A(x+z, t)\right], \quad x \in \mathbf{Z}^{d}, z \in \mathbf{R}^{d}, \quad t=0,1, \ldots,
\end{aligned}
$$

where the $d \times d$ matrix $A(y, t)=\left[A_{i, j}(y, t)\right], y \in \mathbf{R}^{d}, t>0$ is given by the formula

$$
\begin{aligned}
A_{i, j}(y, t) & =u(y, t)+u\left(y+\mathbf{e}_{j}-\mathbf{e}_{i}, t\right)-u\left(y+\mathbf{e}_{j}, t\right)-u\left(y-\mathbf{e}_{i}, t\right) \\
& =-E\left[\frac{\partial^{2} u\left(y+Y_{i, j}, t\right)}{\partial y_{i} \partial y_{j}}\right]
\end{aligned}
$$

with $Y_{i, j}$ the random variable uniformly distributed in the unit square $\left\{y_{j} \mathbf{e}_{j}-y_{i} \mathbf{e}_{i} \in\right.$ $\left.\mathbf{R}^{d}: 0 \leq y_{i}, y_{j} \leq 1\right\}$. It follows then from (4.51), (4.52) that

$$
u(x+z, t+1)-u(x+z, t)+\nabla_{x}^{*} \mathbf{a}_{\mathrm{hom}} \nabla_{x} u(x+z, t)=h_{1}(x+z, t)-h_{2}(x+z, t)
$$

where the functions $h_{j}(\cdot, \cdot), j=1,2$, are given by the formulas

$$
h_{1}(y, t)=E\left[\frac{\partial u(y, t+T)}{\partial t}\right]-\frac{\partial u(y, t)}{\partial t}, \quad y \in \mathbf{R}^{d}, t>0,
$$

$$
h_{2}(y, t)=\sum_{i, j=1}^{d} \mathbf{a}_{\mathrm{hom}}(i, j)\left\{E\left[\frac{\partial^{2} u\left(y+Y_{i, j}, t\right)}{\partial y_{i} \partial y_{j}}\right]-\frac{\partial^{2} u(y, t)}{\partial y_{i} \partial y_{j}}\right\}, y \in \mathbf{R}^{d}, t>0 .
$$


In (4.54) the random variable $T$ is uniformly distributed in the interval $0<T<1$. Since $u(x+z, 0)=f(x+z), x \in \mathbf{Z}^{d}$, we conclude from (4.53) that

$$
\begin{array}{r}
u(x+z, t)=\sum_{y \in \mathbf{Z}^{d}} G_{\mathbf{a}_{\mathrm{hom}}}^{\text {lattice }}(x-y, t) \\
f(y+z)+\sum_{r=1}^{t} \sum_{y \in \mathbf{Z}^{d}} G_{\mathbf{a}_{\mathrm{hom}}}^{\text {lattice }}(x-y, t-r) h_{1}(y+z, r-1) \\
-\sum_{r=1}^{t} \sum_{y \in \mathbf{Z}^{d}} G_{\mathbf{a}_{\mathrm{hom}}}^{\text {lattice }}(x-y, t-r) h_{2}(y+z, r-1) .
\end{array}
$$

Let $Q_{0} \subset \mathbf{R}^{d}$ be the unit cube centered at the origin. Then we have that

$$
\begin{array}{r}
\int_{Q_{0}} d z\left[u(x+z, t)-\sum_{y \in \mathbf{Z}^{d}} G_{\mathbf{a}_{\mathrm{hom}}}^{\text {lattice }}(x-y, t) f(y+z)\right] \\
=\left[G_{\mathbf{a}_{\mathrm{hom}}}(x, t)-G_{\mathbf{a}_{\mathrm{hom}}}^{\text {lattice }}(x, t)\right] \int_{\mathbf{R}^{d}} f(y) d y+\operatorname{Error}(x)
\end{array}
$$

where $|\operatorname{Error}(x)|$ is bounded by the RHS of (4.47).

Next observe from (1.6), (4.54), 4.55) that

$$
\int_{Q_{0}} d z \sum_{y \in \mathbf{Z}^{d}} h_{j}(y+z, t)=0 \quad \text { for } j=1,2 .
$$

It follows from (4.58) that if we integrate the third term on the RHS of (4.56) with respect to $z \in Q_{0}$ it is equal to

$$
\int_{Q_{0}} d z \sum_{r=1}^{t} \sum_{y \in \mathbf{Z}^{d}}\left[G_{\mathbf{a}_{\mathrm{hom}}}^{\text {lattice }}(x-y, t-r)-G_{\mathbf{a}_{\mathrm{hom}}}^{\text {lattice }}(x, t-r)\right] h_{2}(y+z, r-1) .
$$

Using the fact that the distribution of $Y_{j, i}$ is the same as the distribution of $-Y_{i, j}$, we see from (4.55) that $h_{2}(\cdot, t)$ is bounded by the fourth derivative of $u(\cdot, t)$, whence we conclude that there are constants $\gamma, C$ depending only on $d$ such that

$$
\left|h_{2}(y, t)\right| \leq \frac{C \Lambda\|f\|_{\infty}}{[\Lambda t+1]^{(d+4) / 2}} \exp \left[-\frac{\gamma|y|^{2}}{\Lambda t+1}\right], \quad y \in \mathbf{R}^{d}, t>0 .
$$

We also have that there are constants $\gamma, C$ depending only on $d$ such that (4.61)

$$
\left|\nabla G_{\mathbf{a}_{\text {hom }}}^{\text {lattice }}(y, t)\right| \leq \frac{C}{[\Lambda t+1]^{(d+1) / 2}} \exp \left[-\gamma \min \left\{|y|, \frac{|y|^{2}}{\Lambda t+1}\right\}\right], \quad y \in \mathbf{Z}^{d}, t \in \mathbf{Z}^{+} .
$$

Using (4.60), (4.61) we can estimate (4.59) and see that it is bounded by the RHS of (4.47). Since we can do a similar estimate with the function $h_{2}$ replaced by $h_{1}$, we conclude from (4.57) that (4.47) holds. We can obtain the bounds (4.48), (4.49) by taking the gradient of (4.56) with respect to $x$ and following the previous argument. 
The inequality (1.21) of Theorem 1.3 is a consequence of Lemma 4.3 and the following:

Theorem 4.2. Assume Hypothesis 4.1 holds and $4 d \Lambda \leq 1$. Then there exist positive constants $\alpha, \gamma$, with $\alpha \leq 1$, depending only on $d, \Lambda / \lambda$ and a constant $C$ such that for $x \in \mathbf{Z}^{d}, t \in \mathbf{Z}, t \geq 0$,

$\left|\nabla \nabla G_{\mathbf{a}}(x, t)-\nabla \nabla G_{\mathbf{a}_{\text {hom }}}^{\text {lattice }}(x, t)\right| \leq \frac{C}{[\Lambda t+1]^{(d+2+\alpha) / 2}} \exp \left[-\gamma \min \left\{|x|, \frac{|x|^{2}}{\Lambda t+1}\right\}\right]$.

Proof. Let $\chi: \mathbf{R}^{d+1} \rightarrow \mathbf{R}$ be a $C^{\infty}$ function with compact support such that the integral of $\chi(\cdot)$ over $\mathbf{R}^{d+1}$ equals 1 . We write

$$
G_{\mathbf{a}}(x, t)=\chi_{L} * G_{\mathbf{a}}(x, t)+\left[G_{\mathbf{a}}(x, t)-\chi_{L} * G_{\mathbf{a}}(x, t)\right],
$$

where $\chi_{L}(x, t)=\Lambda L^{-(d+2)} \chi\left(x / L, \Lambda t / L^{2}\right), x \in \mathbf{R}^{d}, t \in \mathbf{R}$, and $*$ denotes convolution on $\mathbf{Z}^{d+1}$. Let $\hat{\chi}_{L}(\zeta, \theta), \zeta \in[-\pi, \pi]^{d}, \theta \in[-\pi, \pi]$ be the Fourier transform (2.15) of $\chi_{L}(\cdot, \cdot)$ restricted to the $\mathbf{Z}^{d+1}$ lattice. Since $\chi_{L}(\cdot, \cdot)$ has compact support, $\hat{\chi}_{L}(\cdot, \cdot)$ has an analytic continuation to $\mathbf{C}^{d+1}$. Furthermore for $L \geq 1$ there is a constant $C$ such that

(4.64) $\left|\hat{\chi}_{L}(0,0)-1\right| \leq C / L, \quad\left|\hat{\chi}_{L}\left(\zeta+i a, \theta-i \Lambda|a|^{2}\right)\right| \leq C \exp \left[C|a|^{2} L^{2}\right] \quad a \in \mathbf{R}^{d}$.

There also exist for positive integers $n$ constants $C_{n}$ such that

$$
\left|\hat{\chi}_{L}\left(\zeta+i a, \theta-i \Lambda|a|^{2}\right)\right| \leq \frac{C_{n}}{\left[1+L|\zeta|+L^{2}|\theta| / \Lambda\right]^{n}} \quad \text { if }|a| L \leq 1 .
$$

We assume now that $R<\sqrt{\Lambda t+1}<2 R$ and choose $L=R^{1-\delta}$ for some $\delta>0$. Then from (2.5) we see that

$$
\begin{aligned}
\chi_{L} * & \nabla_{k} \nabla_{j} G_{\mathbf{a}}(x, t) \\
& =\frac{1}{(2 \pi)^{d+1}} \exp \left[a \cdot x / C+\Lambda|a|^{2}(t+1)\right] \int_{[-\pi, \pi]^{d}} \int_{-\pi}^{\pi} f_{a}(\zeta, \theta) d \theta d \zeta,
\end{aligned}
$$

where $a$ is given by (4.23) and $f_{a}(\zeta, \theta)$ is defined by

$$
f_{a}(\zeta, \theta)=\frac{e_{k}(\zeta+i a / C) e_{j}(\zeta+i a / C) \hat{\chi}_{L}\left(\zeta+i a / C, \theta-i \Lambda|a|^{2}\right) e^{-i \zeta . x+i \theta(t+1)}}{e^{\Lambda|a|^{2}+i \theta}-1+e(\zeta-i a / C)^{*} q\left(\zeta+i a / C, \Lambda|a|^{2}+i \theta\right) e(\zeta+i a / C)} .
$$

It follows from Corollary 2.2 and the second inequality of (4.64) that if $|a| L \geq 1$ there is a constant $C_{1}$ such that

$$
\begin{aligned}
& \exp \left[a \cdot x / C+\Lambda|a|^{2}(t+1)\right] \int_{[-\pi, \pi]^{d}} \int_{-\pi}^{\pi}\left|f_{a}(\zeta, \theta)\right| d \theta d \zeta \\
& \leq \frac{C_{1}}{[\Lambda t+1]^{(d+3) / 2}} \exp \left[-\gamma \min \left\{|x|, \frac{|x|^{2}}{\Lambda t+1}\right\}\right] .
\end{aligned}
$$

If $|a| L<1$ we also have from Corollary 2.2 and (4.65), for some constant $C_{1}$, that

$$
\begin{aligned}
\int_{[-\pi, \pi]^{d}} \cap\left\{|\zeta|>1 / R^{1-2 \delta}\right\} & \int_{-\pi}^{\pi}\left|f_{a}(\zeta, \theta)\right| d \theta d \zeta \\
& +\int_{[-\pi, \pi]^{d}} \int_{[-\pi, \pi] \cap\left\{\sqrt{|\theta| / \Lambda}>1 / R^{1-2 \delta}\right\}}\left|f_{a}(\zeta, \theta)\right| d \theta d \zeta \\
\leq & C_{1} /[\Lambda t+1]^{(d+3) / 2} .
\end{aligned}
$$


To estimate the integral of $f_{a}(\zeta, \theta)$ over the set $\left\{(\zeta, \theta):|\zeta|<1 / R^{1-2 \delta}, \sqrt{|\theta| / \Lambda}<\right.$ $\left.1 / R^{1-2 \delta}\right\}$ we use the Hölder continuity (4.9) of the function $q(\cdot, \cdot)$. Thus let $g_{a}(\cdot, \cdot)$ be defined similarly to the function $f_{a}(\cdot, \cdot)$ by

$$
g_{a}(\zeta, \theta)=\frac{e_{k}(\zeta+i a / C) e_{j}(\zeta+i a / C) \hat{\chi}_{L}\left(\zeta+i a / C, \theta-i \Lambda|a|^{2}\right) e^{-i \zeta \cdot x+i \theta(t+1)}}{e^{\Lambda|a|^{2}+i \theta}-1+e(\zeta-i a / C)^{*} q(0,0) e(\zeta+i a / C)} .
$$

Then from (4.9) we see that

$$
\int_{\left\{|\zeta|<1 / R^{1-2 \delta}\right\}} \int_{\sqrt{|\theta| / \Lambda}<1 / R^{1-2 \delta}}\left|f_{a}(\zeta, \theta)-g_{a}(\zeta, \theta)\right| d \theta d \zeta \leq C_{2} /[\Lambda t+1]^{d+2+\alpha)(1-2 \delta)}
$$

for some constant $C_{2}$. We choose now $\delta>0$ in (4.71) sufficiently small so that $(d+2+\alpha)(1-2 \delta)>d+2$. It follows then from (4.68), (4.69), (4.71) that $\mid \chi_{L} *$ $\nabla_{k} \nabla_{j} G_{\mathbf{a}}(x, t)-\chi_{L} * \nabla_{k} \nabla_{j} G_{\mathbf{a}_{\text {hom }}}^{\text {lattice }}(x, t) \mid$ is bounded by the RHS of (4.62).

To complete the proof of the inequality (4.62) we use the Hölder continuity result of [1]. Thus from the first inequality of (4.64) and [11] we see that $\mid \nabla_{k} \nabla_{j} G_{\mathbf{a}}(x, t)-$ $\chi_{L} * \nabla_{k} \nabla_{j} G_{\mathbf{a}}(x, t) \mid$ is bounded by the RHS of (4.62) for some $\alpha>0$. The result follows.

We can essentially repeat the foregoing arguments for the continuous time averaged Green's function $G_{\mathbf{a}}(x, t), x \in \mathbf{Z}^{d}, t \geq 0$, for (1.4). In the continuous time case our hypothesis is:

Hypothesis 4.2. Let $T_{\xi, \eta}$ be the operator (2.47) on the Hilbert space $\mathcal{H}(\Omega)$ and let $T_{\xi, \eta}^{*}$ denote its adjoint. Then for $k \geq 1, p_{2}=p_{3}=\cdots=p_{k}=1$, and $S_{\xi, \eta}=T_{\xi, \eta}$ or $S_{\xi, \eta}=T_{\xi, \eta}^{*}$, there exists $p_{0}(\Lambda / \lambda)>1$ depending only on $d, \Lambda / \lambda$ and a constant $C(k)$ such that

$$
\begin{aligned}
& \left\|\sum_{x_{1}, \ldots x_{k} \in \mathbf{Z}^{d+1}} \int_{\mathbf{R}^{k}} d t_{1} \cdots d t_{k}\left\{\prod_{j=1}^{k} g_{j}\left(x_{j}, t_{j}\right) \tau_{x_{j},-t_{j}} P \mathbf{b}(\cdot)\left[I-P S_{\xi, \eta} \mathbf{b}(\cdot)\right]^{-1}\right\} v\right\| \\
& \leq C(k) \prod_{j=1}^{k}\left\|g_{j}\right\|_{p_{j}}|v| \quad \text { for } g_{j} \in L^{p_{j}}\left(\mathbf{Z}^{d} \times \mathbf{R}, \mathbf{C}^{d} \otimes \mathbf{C}^{d}\right), j=1, \ldots, k, v \in \mathbf{C}^{d},
\end{aligned}
$$

provided $1 \leq p_{1} \leq p_{0}(\Lambda / \lambda)$ and $\xi \in \mathbf{C}^{d}, \eta \in \mathbf{C}$ satisfy $0<\Re \eta<\Lambda,|\Im \xi| \leq$ $C_{1} \sqrt{\Re \eta / \Lambda}$, with $C_{1}$ depending only on $d, \Lambda / \lambda$.

Assuming Hypothesis 4.2 holds, we can prove the analogues of Proposition 4.1, Theorem 4.1 and Theorem 4.2 for the continuous case. Theorem 1.3 therefore follows in the continuous time case once we are able to establish Hypothesis 4.2.

\section{INDEPENDENT VARIABLE ENVIRONMENT}

Our goal in this section will be to prove Hypothesis 3.1 and its generalized form Hypothesis 4.1 in the case when the variables $\mathbf{a}\left(\tau_{x, t}\right), x \in \mathbf{Z}^{d}, t \in \mathbf{Z}$, are independent. Following [9] we first consider the case of a Bernoulli environment. Thus for each $x \in \mathbf{Z}^{d}, t \in \mathbf{Z}$, let $Y_{x, t}$ be independent Bernoulli variables, whence $Y_{x, t}= \pm 1$ with equal probability. The probability space $(\Omega, \mathcal{F}, P)$ is then the space generated by all the variables $Y_{x, t},(x, t) \in \mathbf{Z}^{d+1}$. A point $\omega \in \Omega$ is a set of 
configurations $\left\{\left(Y_{n}, n\right): n \in \mathbf{Z}^{d+1}\right\}$. For $(x, t) \in \mathbf{Z}^{d+1}$ the translation operator $\tau_{x, t}$ acts on $\Omega$ by taking the point $\omega=\left\{\left(Y_{n}, n\right): n \in \mathbf{Z}^{d+1}\right\}$ to $\tau_{x, t} \omega=\left\{\left(Y_{n+(x,-t)}, n\right)\right.$ : $\left.n \in \mathbf{Z}^{d+1}\right\}$. The random matrix $\mathbf{a}(\cdot)$ is then defined by

$$
\mathbf{a}(\omega)=\left(1+\gamma Y_{0}\right) I_{d}, \quad \omega=\left\{\left(Y_{n}, n\right): n \in \mathbf{Z}^{d+1}\right\},
$$

where $0 \leq \gamma<1$.

In [9] we defined for $1 \leq p<\infty$ Fock spaces $\mathcal{F}^{p}\left(\mathbf{Z}^{d+1}\right)$ of complex valued functions and observed that $\mathcal{F}^{2}\left(\mathbf{Z}^{d+1}\right)$ is unitarily equivalent to $L^{2}(\Omega)$. We can similarly define Fock spaces $\mathcal{H}_{\mathcal{F}}^{p}\left(\mathbf{Z}^{d+1}\right)$ of vector valued functions with range $\mathbf{C}^{d}$ such that $\mathcal{H}_{\mathcal{F}}^{2}\left(\mathbf{Z}^{d+1}\right)$ is unitarily equivalent to $\mathcal{H}(\Omega)$. Hence we can regard the operator $T_{\xi, \eta}$ of (2.10) as acting on $\mathcal{H}_{\mathcal{F}}^{2}\left(\mathbf{Z}^{d+1}\right)$, and by unitary equivalence it is a bounded operator satisfying $\left\|T_{\xi, \eta}\right\| \leq 1$ for $\xi \in \mathbf{R}^{d}$, $\Re \eta>0$. From (2.10) we see that $T_{\xi, \eta}$ acts as a convolution operator on $N$ particle wave functions $\psi_{N}(\cdot)$ in $\mathcal{H}_{\mathcal{F}}^{2}\left(\mathbf{Z}^{d+1}\right)$ as

$$
\begin{aligned}
T_{\xi, \eta} \psi_{N}\left(x_{1}, t_{1}, \ldots, x_{N}, t_{N}\right) & \\
=\Lambda \sum_{t^{\prime}=1}^{\infty} e^{-\eta t^{\prime}} \sum_{x \in \mathbf{Z}^{d}}\{ & \left.\nabla \nabla^{*} G_{\Lambda}\left(x^{\prime}, t^{\prime}-1\right)\right\}^{*} \exp \left[-i x^{\prime} \cdot \xi\right] \\
\times & \psi_{N}\left(x_{1}-x^{\prime}, t_{1}-t^{\prime}, . ., x_{N}-x^{\prime}, t_{N}-t^{\prime}\right) .
\end{aligned}
$$

Note that for all $N$ particle wave functions, $T_{\xi, \eta}$ acts as a convolution operator on functions on $\mathbf{Z}^{d+1}$. Hence its action is determined by its action on 1-particle wave functions. Let $\hat{\psi}_{1}(\zeta, \theta), \zeta \in[-\pi, \pi]^{d}, \theta \in[-\pi, \pi]$, be the Fourier transform (2.15) of the 1-particle wave function $\psi_{1}(x, t), x \in \mathbf{Z}^{d}, t \in \mathbf{Z}$. We see from (15.2) that for $\xi \in \mathbf{C}^{d}, \Re \eta>0$, the action of $T_{\xi, \eta}$ in Fourier space is given by

$\hat{T}_{\xi, \eta} \hat{\psi}_{1}(\zeta, \theta)=\frac{\Lambda e(\xi-\zeta) e(\bar{\xi}-\zeta)^{*}}{e^{\eta-i \theta}-1+\Lambda e(\bar{\xi}-\zeta)^{*} e(\xi-\zeta)} \hat{\psi}_{1}(\zeta, \theta), \quad \zeta \in[-\pi, \pi]^{d}, \theta \in[-\pi, \pi]$.

Hence the result of Lemma 2.1 for the Bernoulli case follows from:

Lemma 5.1. Assume $4 d \Lambda \leq 1$. Then there exist positive constants $C_{1}, C_{2}$ depending only on $d$ such that for $(\xi, \eta)$ in the region $\left\{(\xi, \eta) \in \mathbf{C}^{d+1}: 0<\Re \eta<\Lambda,|\Im \xi|<\right.$ $\left.C_{1} \sqrt{\Re \eta / \Lambda}\right\}$, there is the inequality

$$
\Lambda \max \left[|e(\xi)|^{2},|e(\bar{\xi})|^{2}\right] \leq\left(1+C_{2}|\Im \xi|^{2} /[\Re \eta / \Lambda]\right)\left|e^{\eta}-1+\Lambda e(\bar{\xi})^{*} e(\xi)\right| .
$$

Proof. We have that

$$
\begin{aligned}
\left|e^{\eta}-1+\Lambda e(\bar{\xi})^{*} e(\xi)\right| & \geq e^{\Re \eta}-\left|1-\Lambda e(\bar{\xi})^{*} e(\xi)\right| \\
& \geq e^{\Re \eta}-1+\Lambda e(\Re \xi)^{*} e(\Re \xi)-\Lambda\left|e(\Re \xi)^{*} e(\Re \xi)-e(\bar{\xi})^{*} e(\xi)\right|,
\end{aligned}
$$

where we have used the fact that $4 d \Lambda \leq 1$. Observe that there is a constant $C$ depending only on $d$ such that

$$
\begin{aligned}
\Lambda\left|e(\Re \xi)^{*} e(\Re \xi)-e(\bar{\xi})^{*} e(\xi)\right| \leq C \Lambda\left[|\Im \xi|^{2}+|\Im \xi||e(\Re \xi)|\right] \\
\leq C^{2}\left\{\Lambda e(\Re \xi)^{*} e(\Re \xi)\right\}|\Im \xi|^{2} /[\Re \eta / \Lambda]+\left[1 / 4+C C_{1}^{2}\right] \Re \eta .
\end{aligned}
$$

We conclude then from (5.5), (5.6) that

$$
\left|e^{\eta}-1+\Lambda e(\bar{\xi})^{*} e(\xi)\right| \geq\left[3 / 4-C C_{1}^{2}\right] \Re \eta+\left[1-C^{2}|\Im \xi|^{2} /[\Re \eta / \Lambda] \Lambda e(\Re \xi)^{*} e(\Re \xi) .\right.
$$


The inequality (5.4) follows from (5.7) by observing similarly to (5.6) that

$$
\Lambda|e(\xi)|^{2} \leq \Lambda e(\Re \xi)^{*} e(\Re \xi)+C \Lambda\left[|\Im \xi|^{2}+|\Im \xi||e(\Re \xi)|\right] .
$$

Lemma 5.2. Assume $4 d \Lambda \leq 1$. Then there exist positive constants $C_{1}, C_{2}, C_{3}$ depending only on $d$ such that for $(\xi, \eta)$ in the region $\left\{(\xi, \eta) \in \mathbf{C}^{d+1}: 0<\Re \eta<\right.$ $\left.\Lambda,|\Im \xi|<C_{1} \sqrt{\Re \eta / \Lambda}\right\}$, the operator $T_{\xi, \eta}$ of (5.2) is bounded on $\mathcal{H}_{\mathcal{F}}^{p}\left(\mathbf{Z}^{d+1}\right)$ for $p=3 / 2$ or $p=3$, and the norm $\left\|T_{\xi, \eta}\right\|_{p}$ of $T_{\xi, \eta}$ satisfies the inequality $\left\|T_{\xi, \eta}\right\|_{p} \leq$ $C_{3}\left(1+C_{2}|\Im \xi|^{2} /[\Re \eta / \Lambda]\right)$.

Proof. It will be sufficient for us to prove the theorem on the space of 1-particle wave functions. To do this we follow the argument of Jones [18, which adapts the methodology of Calderon-Zygmund $[5]$ to Fourier multipliers associated with parabolic PDE. A more general theory of Fourier multipliers can be found in Chapter IV of [30], but because of the generality it is hard to estimate the values of constants using this theory.

For a set $E \subset \mathbf{Z}^{d+1}$, we denote by $|E|$ the number of lattice points of $\mathbf{Z}^{d+1}$ contained in $E$. Let $\psi(x, t), x \in \mathbf{Z}^{d}, t \in \mathbf{Z}$, be a 1-particle wave function with finite support. We shall show that for any $\gamma>0$, the set $E_{\gamma}=\left\{(x, t) \in \mathbf{Z}^{d+1}\right.$ : $\left.\left|T_{\xi, \eta} \psi(x, t)\right|>\gamma\right\}$ satisfies the inequality

$$
\left|E_{\gamma}\right| \leq C_{4}\left(1+C_{2}|\Im \xi|^{2} /[\Re \eta / \Lambda]\right) \gamma^{-2} \sum_{(x, t) \in \mathbf{Z}^{d+1}} \min [|\psi(x, t)|, \gamma]^{2}+C_{5} \beta^{\psi}(\gamma)
$$

where $C_{2}$ is the constant of Lemma 5.1 and $C_{4}, C_{5}$ depend only on $d$. The function $\beta^{\psi}(\cdot)$ is defined in [5, 18, in terms of the distribution function of $\psi(\cdot, \cdot)$. Once (5.9) is proved the result follows from the argument of [5], which shows that $\left\|T_{\xi, \eta}\right\|_{p}$ is simply bounded in terms of the constants occurring in (5.9).

We use a Calderon-Zygmund decomposition to prove (5.9). Recalling that $1 / \Lambda \geq$ $4 d$, let $N_{0} \geq 2$ be the integer which satisfies $2^{N_{0}} \leq 1 / \Lambda<2^{N_{0}+1}$. We choose $a_{1}, \ldots a_{d}, b \in \mathbf{Z}$ and sufficiently large integer $N_{1}$ such that the rectangle $R=$ $\left\{(x, t)=\left(x_{1}, \ldots, x_{d}, t\right) \in \mathbf{R}^{d+1}: a_{j}+1 / 2 \leq x_{j} \leq 2^{N_{1}}+a_{j}+1 / 2, j=\right.$ $1, \ldots, d$, and $\left.b+1 / 2 \leq t \leq 2^{2 N_{1}+N_{0}}+b+1 / 2\right\}$ contains the support of $\psi(\cdot, \cdot)$ and

$$
\frac{1}{|R|} \sum_{(x, t) \in R \cap \mathbf{Z}^{d+1}}|\psi(x, t)| \leq \gamma .
$$

Note that the length of the side of $R$ in the $t$ direction is $2^{N_{0}}$ times the square of the length of a side in an $x_{j}$ direction for all $1 \leq j \leq d$. We subdivide $R$ into $2^{d} \times 4$ subrectangles with the same property and continue to similarly subdivide until we reach a set of disjoint rectangles $R_{m}, m=1, \ldots, M_{1}$, with side in the $x_{j}, 1 \leq j \leq d$, direction a non-negative power of 2 , which satisfy the inequality

$$
\gamma<\frac{1}{\left|R_{m}\right|} \sum_{(x, t) \in R_{m}}|\psi(x, t)| \leq 2^{d+2} \gamma, \quad 1 \leq m \leq M_{1},
$$

together with a set of rectangles $R_{m}^{\prime}, m=1,2, \ldots, M_{2}$, with side in the $x_{j}, 1 \leq$ $j \leq d$, direction equal to 1 and equal to $2^{N_{0}}$ in the $t$ direction which satisfy

$$
\frac{1}{\left|R_{m}^{\prime}\right|} \sum_{(x, t) \in R_{m}^{\prime}}|\psi(x, t)| \leq \gamma \text {. }
$$


We subdivide the rectangles $R_{m}^{\prime}, m=1, \ldots, M_{2}$, into 2 rectangles with side in the $t$ direction of length $2^{N_{0}-1}$, and continue to subdivide until we reach a set of disjoint rectangles $R_{m}, m=M_{1}+1, \ldots, M$, with side in the $t$ direction a non-negative power of 2 , which satisfy the inequality

$$
\gamma<\frac{1}{\left|R_{m}\right|} \sum_{(x, t) \in R_{m}}|\psi(x, t)| \leq 2 \gamma, \quad M_{1}+1 \leq m \leq M,
$$

together with a set of unit cubes centered at lattice points of $\mathbf{Z}^{d+1}$. Setting $D_{\gamma}=$ $\cup_{m=1}^{M} R_{m}$, one sees that $\mathbf{R}^{d+1}-D_{\gamma}$ is a union of unit cubes centered at lattice points of $\mathbf{Z}^{d+1}$, whence

$$
|\psi(x, t)| \leq \gamma \quad \text { for }(x, t) \in \mathbf{Z}^{d+1} \cap\left[\mathbf{R}^{d+1}-D_{\gamma}\right] .
$$

We consider the distribution function $\gamma \rightarrow\left|\left\{(x, t) \in \mathbf{Z}^{d+1}:|\psi(x, t)|>\gamma\right\}\right|$ of $\psi(\cdot, \cdot)$ with domain $\{\gamma \geq 0\}$, which is a piece-wise constant right continuous decreasing function with range $0 \leq s \leq|\operatorname{supp}[\psi(\cdot, \cdot)]|$. The decreasing rearrangement $\psi^{*}(s)$ of $\psi(\cdot, \cdot)$ with domain $s \geq 0$ is also a piece-wise constant right continuous decreasing function satisfying $\psi^{*}(0)=\sup |\psi(\cdot, \cdot)|$ and $\psi^{*}(s)=0$ for $s \geq|\operatorname{supp}[\psi(\cdot, \cdot)]|$. It is the approximate right continuous inverse of the distribution function for $0 \leq s \leq|\operatorname{supp}[\psi(\cdot, \cdot)]|$. In view of (5.11), (5.13) we have that

$$
\gamma<\frac{1}{\left|D_{\gamma}\right|} \sum_{(x, t) \in D_{\gamma}}|\psi(x, t)| \leq \frac{1}{\left|D_{\gamma}\right|} \int_{0}^{\left|D_{\gamma}\right|} \psi^{*}(s) d s=\beta_{\psi}\left(\left|D_{\gamma}\right|\right),
$$

where the function $\beta_{\psi}(s)$ with domain $s \geq 0$ is decreasing and continuous with range $0<\gamma \leq \sup |\psi(\cdot, \cdot)|$. There is a well-defined inverse function $\beta^{\psi}(\gamma)$ for $\beta_{\psi}(\cdot)$ with domain $0<\gamma<\sup |\psi(\cdot, \cdot)|$, and (5.15) implies that $\left|D_{\gamma}\right| \leq \beta^{\psi}(\gamma)$.

We write $\psi(\cdot, \cdot)=\psi_{1}(\cdot, \cdot)+\psi_{2}(\cdot, \cdot)$, where the function $\psi_{1}(\cdot, \cdot)$ is defined by

$$
\begin{array}{r}
\psi_{1}(x, t)=\frac{1}{\left|R_{m}\right|} \sum_{\left(x^{\prime}, t^{\prime}\right) \in R_{m}} \psi\left(x^{\prime}, t^{\prime}\right) \quad \text { if }(x, t) \in R_{m} \text { for some } m, 1 \leq m \leq M \\
\psi_{1}(x, t)=\psi(x, t) \quad \text { otherwise } .
\end{array}
$$

From Lemma 5.1 and (5.14) we have then that

$$
\begin{aligned}
& \left|\left\{(x, t) \in \mathbf{Z}^{d+1}:\left|T_{\xi, \eta} \psi_{1}(x, t)\right|>\gamma / 2\right\}\right| \\
& \leq\left(1+C_{2}|\Im \xi|^{2} /[\Re \eta / \Lambda]\right)^{2}\left\{4 \gamma^{-2} \sum_{(x, t) \in \mathbf{Z}^{d+1}} \min [|\psi(x, t)|, \gamma]^{2}+2^{2 d+6}\left|D_{\gamma}\right|\right\} .
\end{aligned}
$$

To bound the distribution function of $\psi_{2}(\cdot, \cdot)$ which has support contained in $D_{\gamma}$, we consider a rectangle $R_{m}, 1 \leq m \leq M$, with center $\left(x^{m}, t^{m}\right) \in \mathbf{Z}^{d+1}$ and let $\tilde{R}_{m}$ be the double of $R_{m}$. We observe that similarly to $(2.12)$ there is a constant $C_{d}$ depending only on $d$ such that the function $\nabla \nabla^{*} G_{\Lambda}(x, t)$ satisfies inequalities

$$
\begin{aligned}
\left|e^{-\Re \eta(t+1)-i x \cdot \Im \xi} \nabla \nabla^{*} G_{\Lambda}(x, t+1)-e^{-\Re \eta t-i x \cdot \Im \xi} \nabla \nabla^{*} G_{\Lambda}(x, t)\right| & \\
\leq & \frac{C_{d}}{(t+1)[\Lambda t+1]^{d / 2+1}} \exp \left[-\frac{\min \left\{|x|,|x|^{2} /(\Lambda t+1)\right\}}{C_{d}}\right]
\end{aligned}
$$




$$
\begin{aligned}
& \left|e^{-\Re \eta t-i\left(x+\mathbf{e}_{j}\right) \cdot \Im \xi} \nabla \nabla^{*} G_{\Lambda}\left(x+\mathbf{e}_{j}, t\right)-e^{-\Re \eta t-i x \cdot \Im \xi} \nabla \nabla^{*} G_{\Lambda}(x, t)\right| \\
& \quad \leq \frac{C_{d}}{[\Lambda t+1]^{d / 2+3 / 2}} \exp \left[-\frac{\min \left\{|x|,|x|^{2} /(\Lambda t+1)\right\}}{C_{d}}\right], j=1, \ldots, d,
\end{aligned}
$$

provided $\xi \in \mathbf{C}^{d}, \eta \in \mathbf{C}$, satisfy the conditions in the statement of the lemma. Extending the function $G_{\Lambda}(x, t), x \in \mathbf{Z}^{d}, t=0,1,2, \ldots$, defined by (2.11) to get domain $\mathbf{Z}^{d+1}$ by setting $G_{\Lambda}(x, t)=0$ for $x \in \mathbf{Z}^{d}, t<0$, we conclude from (5.18), (5.19) that if $\left(x^{\prime}, t^{\prime}\right) \in R_{m}$, then there is a constant $C_{d}$ depending only on $d$ such that

$$
\begin{aligned}
\sum_{(x, t) \in \mathbf{Z}^{d+1}-\tilde{R}_{m}} \Lambda \mid e^{-\Re \eta\left(t-t^{\prime}\right)-i\left(x-x^{\prime}\right) \cdot \Im \xi} \nabla \nabla^{*} G_{\Lambda}\left(x-x^{\prime}, t-t^{\prime}\right) \\
-e^{-\Re \eta\left(t-t^{m}\right)-i\left(x-x^{m}\right) \cdot \Im \xi} \nabla \nabla^{*} G_{\Lambda}\left(x-x^{m}, t-t^{m}\right) \mid \leq C_{d} .
\end{aligned}
$$

It follows from (5.11), (5.13), (5.20) that if $\tilde{D}_{\gamma}=\bigcup_{m=1}^{M} \tilde{R}_{m}$, then

$$
\sum_{(x, t) \in \mathbf{Z}^{d+1}-\tilde{D}_{\gamma}}\left|T_{\xi, \eta} \psi_{2}(x, t)\right| \leq C_{d} \gamma\left|D_{\gamma}\right|
$$

for some constant $C_{d}$ depending only on $d$. Hence we have that

$\left|\left\{(x, t) \in \mathbf{Z}^{d+1}:\left|T_{\xi, \eta} \psi_{2}(x, t)\right|>\gamma / 2\right\}\right| \leq 2 C_{d}\left|D_{\gamma}\right|+\left|\tilde{D}_{\gamma}\right| \leq\left[2 C_{d}+2^{d+2}\right]\left|D_{\gamma}\right|$.

The inequality (5.9) follows from (5.17) and (5.22).

Corollary 5.1. Under the assumptions of Lemma 5.2 the operator $T_{\xi, \eta}$ is bounded on $\mathcal{H}^{p}\left(\mathbf{Z}^{d+1}\right)$ for $3 / 2 \leq p \leq 3$ and $\left\|T_{\xi, \eta}\right\|_{p} \leq[1+\delta(p)]\left(1+C_{2}|\Im \xi|^{2} /[\Re \eta / \Lambda]\right)$, where the function $\delta(\cdot)$ depends only on $d$ and $\lim _{p \rightarrow 2} \delta(p)=0$.

Proof. The result follows from Lemma 5.1, Lemma 5.2 and the Riesz-Thorin interpolation theorem [31].

Proof of Hypothesis 4.1. We choose $q_{0}=q_{0}(\Lambda / \lambda)$ with $1<q_{0}<2$ so that $\delta\left(q_{0}\right) \leq$ $\lambda / 2 \Lambda$, where $\delta(\cdot)$ is the function in the statement of Corollary 5.1. It follows then from Young's inequality that Hypothesis 4.1 holds if we choose $p_{0}=p_{0}(\Lambda / \lambda)>$ 1 with $1 / p_{0}+1 / q_{0}=3 / 2$. It is shown in 9 . how to extend the argument for the Bernoulli environment corresponding to (5.1) to general i.i.d. environments $\mathbf{a}\left(\tau_{x, t^{*}}\right),(x, t) \in \mathbf{Z}^{d+1}$. We have therefore proven Hypothesis 4.1 for $\mathbf{a}\left(\tau_{x, t}\right),(x, t) \in$ $\mathbf{Z}^{d+1}$, i.i.d. such that (1.1) holds.

\section{MASsive FIELD THEORY ENVIRONMENT}

In this section we show that Hypothesis 3.2 and its generalization Hypothesis 4.2 hold if $(\Omega, \mathcal{F}, P)$ is given by the massive field theory environment determined by (1.10), (1.11). We recall the main features of the construction of this measure. Let $L$ be a positive even integer and $Q=Q_{L} \subset \mathbf{Z}^{d}$ be the integer lattice points in the cube centered at the origin with side of length $L$. By a periodic function $\phi: Q \times \mathbf{R} \rightarrow \mathbf{R}$ we mean a function $\phi$ on $Q \times \mathbf{R}$ with the property that $\phi(x, t)=\phi(y, t)$ for all $x, y \in Q, t \in \mathbf{R}$, such that $x-y=L \mathbf{e}_{k}$ for some $k, 1 \leq k \leq d$. Let $\Omega_{Q}$ be the space of continuous in time periodic functions $\phi: Q \times \mathbf{R} \rightarrow \mathbf{R}$ and $\mathcal{F}_{Q}$ be the Borel algebra generated by the requirement that the functions $\phi(\cdot, \cdot) \rightarrow \phi(x, t)$ from $\Omega_{Q} \rightarrow \mathbf{R}$ are Borel measurable for all $x \in Q$ and $t$ rational. For $m>0$ we define a 
probability measure $P_{Q}$ on $\left(\Omega_{Q}, \mathcal{F}_{Q}\right)$ by first defining expectations of functions of the variables $\phi(x, 0), x \in Q$, as follows:

(6.1) $\langle F(\phi(\cdot, 0))\rangle_{\Omega_{Q}}$

$$
=\int_{\mathbf{R}^{L^{d}}} F(\phi(\cdot)) \exp \left[-\sum_{x \in Q}\left\{V(\nabla \phi(x))+\frac{1}{2} m^{2} \phi(x)^{2}\right\}\right] \prod_{x \in Q} d \phi(x) / \text { normalization, }
$$

where $F: \mathbf{R}^{L^{d}} \rightarrow \mathbf{R}$ is a continuous function such that $|F(z)| \leq C \exp [A|z|], z \in$ $\mathbf{R}^{L^{d}}$, for some constants $C, A$. By translation invariance of the measure (6.1) we see that $\langle\phi(x, 0)\rangle_{\Omega_{Q}}=0$ for all $x \in Q$ and hence the Brascamp-Lieb inequality [2] applied to (6.1) and function $F(\phi(\cdot))=\exp [(f, \phi)]$, where $(\cdot, \cdot)$ is the Euclidean inner product for periodic functions on $Q$, yields the inequality

$$
\langle\exp [(f, \phi)]\rangle_{\Omega_{Q}} \leq \exp \left[\frac{1}{2}\left(f,\left\{-\lambda \Delta+m^{2}\right\}^{-1} f\right)\right] .
$$

The variables $\phi(x, t), x \in Q, t>0$, are determined from the variables $\phi(x, 0), x \in$ $Q$, by solving the stochastic differential equation

$$
d \phi(x, t)=-\frac{\partial}{\partial \phi(x, t)} \sum_{x^{\prime} \in Q} \frac{1}{2}\left\{V\left(\nabla \phi\left(x^{\prime}, t\right)\right)+m^{2} \phi\left(x^{\prime}, t\right)^{2} / 2\right\} d t+d B(x, t)
$$

where $x \in Q$ and $t>0$, and where $B(x, \cdot), x \in Q$, are independent copies of Brownian motion modulo the periodicity constraint on $Q$. Since (6.1) is the invariant measure for the stochastic process $\phi(\cdot, t), t \geq 0$, it follows that (6.1), (6.3) determine a stationary process for $t \geq 0$, which therefore can be extended to all $t \in \mathbf{R}$. Furthermore the functions $t \rightarrow \phi(x, t)$ on $\mathbf{R}$ are continuous with probability 1 for all $x \in Q$. The probability measure $P_{Q}$ on $\left(\Omega_{Q}, \mathcal{F}_{Q}\right)$ is the measure induced by the stationary process $\phi(\cdot, t), t \in \mathbf{R}$.

The probability space $(\Omega, \mathcal{F}, P)$ on continuous in time fields $\phi: \mathbf{Z}^{d} \times \mathbf{R} \rightarrow \mathbf{R}$ is obtained as the limit of the spaces $\left(\Omega_{Q}, \mathcal{F}_{Q}, P_{Q}\right)$ as $|Q| \rightarrow \infty$. In particular one has from Lemma 2.4 of [7] the following result:

Proposition 6.1. Assume $m>0$ and let $F: \mathbf{R}^{k} \rightarrow \mathbf{R}$ be a $C^{1}$ function which satisfies the inequality

$$
|D F(z)| \leq A \exp [B|z|], \quad z \in \mathbf{R}^{k},
$$

for some constants $A, B$. Then for any $x_{1}, \ldots, x_{k} \in \mathbf{Z}^{d}$, and $t_{1}, \ldots, t_{k} \in \mathbf{R}$, the limit

$$
\begin{gathered}
\lim _{|Q| \rightarrow \infty}\left\langle F\left(\phi\left(x_{1}, t_{1}\right), \phi\left(x_{2}, t_{2}\right), \ldots, \phi\left(x_{k}, t_{k}\right)\right)\right\rangle_{\Omega_{Q}} \\
=\left\langle F\left(\phi\left(x_{1}, t_{1}\right), \phi\left(x_{2}, t_{2}\right), \ldots, \phi\left(x_{k}, t_{k}\right)\right)\right\rangle
\end{gathered}
$$

exists and is finite.

From (6.2) and the Helly-Bray theorem [3, 13] one sees that Proposition 6.1 implies the existence of a unique Borel probability measure on $\mathbf{R}^{k}$ corresponding to the probability distribution of the variables $\left(\phi\left(x_{1}, t_{1}\right), \ldots, \phi\left(x_{k}, t_{k}\right)\right) \in \mathbf{R}^{k}$, and this measure satisfies (6.5). The Kolmogorov construction [3, 13, then implies the existence of a Borel measure on fields $\phi: \mathbf{Z}^{d} \times \mathbf{R} \rightarrow \mathbf{R}$ with finite dimensional distribution functions satisfying (6.5). We have constructed the probability space $(\Omega, \mathcal{F}, P)$ corresponding to (1.10), (1.11) for which $\Omega$ is the set of continuous in 
time functions $\phi: \mathbf{Z}^{d} \times \mathbf{R} \rightarrow \mathbf{R}$, and it is clear that the translation operators $\tau_{x, t}, x \in \mathbf{Z}^{d}, t \in \mathbf{R}$, are measure preserving and form a group.

The BL inequality [2] plays a crucial role in establishing the existence of the limit (6.5) in [7,14. In particular it yields a Poincaré inequality for the measure (6.1). Thus if $F: \mathbf{R}^{L^{d}} \rightarrow \mathbf{R}$ is a $C^{1}$ function such that $|D F(z)| \leq C \exp [A|z|], z \in \mathbf{R}^{L^{d}}$, for some constants $C, A$, then

$$
\operatorname{var}_{\Omega_{Q}}[F(\phi(\cdot, 0))]=\left\langle[F(\phi(\cdot, 0))-\langle F(\phi(\cdot, 0))\rangle]^{2}\right\rangle_{\Omega_{Q}} \leq \frac{1}{m^{2}}\left\langle\|d F(\phi(\cdot, 0))\|^{2}\right\rangle_{\Omega_{Q}},
$$

where $d F(\phi(\cdot, 0)) \in \mathbf{R}^{L^{d}}$ is the gradient of $F$ at $\phi(\cdot, 0)$. A simple proof of (6.6) follows from the Helffer-Sjöstrand (HS) representation [17]:

$$
\begin{aligned}
& \left\langle F_{1}(\phi(\cdot, 0)) F_{2}(\phi(\cdot, 0))\right\rangle_{\Omega_{Q}} \\
& \quad=\left\langle d F_{1}(\phi(\cdot, 0))\left[d^{*} d+\nabla^{*} V^{\prime \prime}(\nabla \phi(\cdot)) \nabla+m^{2}\right]^{-1} d F_{2}(\phi(\cdot, 0))\right\rangle_{\Omega_{Q}},
\end{aligned}
$$

which holds for $C^{1}$ functions $F_{1}, F_{2}: \mathbf{R}^{L^{d}} \rightarrow \mathbf{R}$ that satisfy $\left|F_{j}(z)\right|+\left|D F_{j}(z)\right| \leq$ $C \exp [A|z|], z \in \mathbf{R}^{L^{d}}, j=1,2$, for some constants $C, A$, and $\left\langle F_{1}(\phi(\cdot, 0))\right\rangle_{\Omega_{Q}}=0$. In (6.7) the operator $d^{*}$ is the adjoint of the gradient operator $d$ with respect to the measure (6.1), and hence $d^{*} d$ is a non-negative self-adjoint operator.

Our first goal here will be to prove strong mixing of the operator $\tau_{\mathbf{e}_{1}, 0}$ on $(\Omega, \mathcal{F}, P)$. In order to do this we will need a Poincaré inequality for the measure $\left(\Omega_{Q}, \mathcal{F}_{Q}, P_{Q}\right)$, in particular a generalization of (6.6) to functions $F\left(\phi\left(\cdot, t_{1}\right), \ldots\right.$, $\left.\phi\left(\cdot, t_{k}\right)\right)$ depending on values of the field $\phi(\cdot, \cdot)$ at different times. To do this we follow the development of Gourcy-Wu [16, who make use of the Malliavin calculus [25] to prove a log-Sobolev inequality for such measures. The basic insight of the Malliavin calculus is that the Wiener space generated by independent Brownian motions $B(x, t), x \in Q, t>0$, can be identified with a probability space whose set of configurations is the Hilbert space $L^{2}\left(Q \times \mathbf{R}^{+}\right)$, where $\mathbf{R}^{+}$is the open interval $(0, \infty)$. We denote the Euclidean inner product on $L^{2}\left(Q \times \mathbf{R}^{+}\right)$by $[\cdot, \cdot]$. The measure on $L^{2}\left(Q \times \mathbf{R}^{+}\right)$is uniquely determined by the requirement that the variables $\psi \rightarrow\left[\psi, \psi_{j}\right], j=1, \ldots, k$, are i.i.d. standard normal for any set of orthonormal vectors $\psi_{j}, j=1, \ldots, k$. We denote this Malliavin probability space by $\left(\Omega_{Q, \text { Mal }}, \mathcal{F}_{Q, \text { Mal }}, P_{Q, \text { Mal }}\right)$, where $\Omega_{Q, \text { Mal }}=L^{2}\left(Q \times \mathbf{R}^{+}\right)$and $\mathcal{F}_{Q, \text { Mal }}$ is determined by the requirement that the functions $\psi \rightarrow\left[\psi, \psi_{0}\right]$ from $\Omega_{Q, \text { Mal }}$ to $\mathbf{R}$ are Borel measurable for all $\psi_{0} \in L^{2}\left(Q \times \mathbf{R}^{+}\right)$.

The identification of the Wiener space with $\left(\Omega_{Q, \text { Mal }}, \mathcal{F}_{Q, \text { Mal }}, P_{Q, \text { Mal }}\right)$ follows from the fact that the expectation of a function $F(\psi(\cdot, \cdot))$ with respect to $\left(\Omega_{Q \text {, Mal }}, \mathcal{F}_{Q, \text { Mal }}\right.$, $\left.P_{Q, \text { Mal }}\right)$ is the same as the expectation of $F(W(\cdot, \cdot))$ with respect to Wiener space, where $W(\cdot, \cdot)$ is the white noise process corresponding to $B(\cdot, \cdot)$ in (6.3). Hence the identification may be summarized as follows:

$$
\psi(x, t) \leftrightarrow W(x, t), \quad W(x, t)=d B(x, t) / d t, \quad x \in Q, t>0 .
$$

For $t>0$ let $\mathcal{F}_{t}$ be the $\sigma$-field generated by the Brownian motions $B(x, s), x \in$ $Q, s<t$, of (6.3), so from (6.8) we can regard $\mathcal{F}_{t}$ as a sub $\sigma$-field of $\mathcal{F}_{Q, \text { Mal }}$. We consider next vector fields $G: L^{2}\left(Q \times \mathbf{R}^{+}\right) \rightarrow L^{2}\left(Q \times \mathbf{R}^{+}\right)$on $\Omega_{Q \text {,Mal }}$, which are measurable in the sense that for any $\psi_{0} \in L^{2}\left(Q \times \mathbf{R}^{+}\right)$the function $\psi(\cdot, \cdot) \rightarrow$ $\left[G(\psi(\cdot, \cdot)), \psi_{0}\right]$ is $\left(\Omega_{Q, \text { Mal }}, \mathcal{F}_{Q, \text { Mal }}\right)$ measurable. The vector field is predictable if for any $t, 0<t<\infty, \psi_{0}$ has support in the interval $Q \times[0, t]$ implying that the function $\left[G(\psi(\cdot, \cdot)), \psi_{0}\right]$ is $\mathcal{F}_{t}$ measurable. The Martingale representation theorem 
25] implies that for any function $F \in L^{2}\left(\Omega_{Q, \text { Mal }}\right)$ there is a predictable vector field $G: L^{2}\left(Q \times \mathbf{R}^{+}\right) \rightarrow L^{2}\left(Q \times \mathbf{R}^{+}\right)$such that

$$
\begin{aligned}
\operatorname{var}_{\Omega_{Q, \mathrm{Mal}}}[F(\cdot)] & =\left\langle\|G(\cdot)\|^{2}\right\rangle_{\Omega_{Q, \mathrm{Mal}}}, \\
F(\psi(\cdot, \cdot))-\langle F(\cdot)\rangle & =[G(\psi(\cdot, \cdot)), \psi(\cdot, \cdot)] .
\end{aligned}
$$

Suppose now that $F \in L^{2}\left(\Omega_{Q, \text { Mal }}\right)$ also has a Malliavin derivative $D_{\text {Mal }} F$ : $L^{2}\left(Q \times \mathbf{R}^{+}\right) \rightarrow L^{2}\left(Q \times \mathbf{R}^{+}\right)$with the property that $\left\langle\left\|D_{\mathrm{Mal}} F(\cdot)\right\|^{2}\right\rangle_{\Omega_{Q . \mathrm{Mal}}}<\infty$. The Clark-Ocone formula 25] states that the vector field $G(\psi(\cdot, \cdot))$ in (6.9) can be expressed in terms of the Malliavin derivative $D_{\mathrm{Mal}} F(\psi(\cdot, \cdot))$. Denoting the values of $G(\psi(\cdot, \cdot)), D_{\mathrm{Mal}} F(\psi(\cdot, \cdot))$ at $(x, t) \in Q \times \mathbf{R}^{+}$by $G(x, t ; \psi(\cdot, \cdot)), D_{\mathrm{Mal}} F(x, t ; \psi(\cdot, \cdot))$ respectively, then

$$
G(x, t ; \psi(\cdot, \cdot))=\left\langle D_{\mathrm{Mal}} F(x, t ; \psi(\cdot, \cdot)) \mid \mathcal{F}_{t}\right\rangle_{\Omega_{Q, \mathrm{Mal}}} \quad x \in Q, t>0 .
$$

We show how the Clark-Ocone formula (6.9), (6.10) implies the HS formula (6.7). Let $\phi(\cdot, T)$ be the solution at time $T>0$ of (6.3) with initial data $\phi(\cdot, 0)=0$ and $f: Q \rightarrow \mathbf{R}$. We can find an expression for the Malliavin derivative of the function $F(\psi(\cdot, \cdot))=(f(\cdot), \phi(\cdot, T))$ by analyzing the first variation equation for (6.3). Evidently one has that $D_{\mathrm{Mal}} F(x, t ; \psi(\cdot, \cdot))=0$ for $x \in Q, t>T$. To get an expression for $D_{\mathrm{Mal}} F(x, t ; \psi(\cdot, \cdot))$ when $t \leq T$ we first note from (6.3) that

$$
\begin{aligned}
\frac{d}{d t}(f(\cdot), \phi(\cdot, t))= & -\frac{1}{2}\left\{\left(\nabla f(\cdot), V^{\prime}(\nabla \phi(\cdot, t))\right)+m^{2}(f(\cdot), \phi(\cdot, t))\right\} \\
& +(f(\cdot), W(\cdot, t)), \quad t>0 .
\end{aligned}
$$

It follows from (6.11) that for $\psi_{0} \in L^{2}\left(Q \times \mathbf{R}^{+}\right)$the function $\left.\xi(\cdot, t)=\left[D_{\mathrm{Mal}} \phi(\cdot, t)\right), \psi_{0}\right]$ from $Q$ to $\mathbf{R}$ is a solution to the initial value problem

$$
\begin{aligned}
& \frac{d}{d t}(f(\cdot), \xi(\cdot, t))=-\frac{1}{2}\left\{\left(\nabla f(\cdot), V^{\prime \prime}(\nabla \phi(\cdot, t)) \nabla \xi(\cdot, t)\right)\right. \\
& \left.+m^{2}(f(\cdot), \xi(\cdot, t))\right\}+\left(f(\cdot), \psi_{0}(\cdot, t)\right) \text { for } t>0, f: Q \rightarrow \mathbf{R} ; \quad \xi(\cdot, 0)=0 .
\end{aligned}
$$

From (6.12) we see that $\xi(x, t), x \in Q, t>0$, is the solution to the initial value problem for the parabolic PDE:

$$
\begin{aligned}
\frac{\partial \xi(x, t)}{\partial t} & =-\frac{1}{2}\left\{\nabla^{*} V^{\prime \prime}(\nabla \phi(x, t)) \nabla \xi(x, t)+m^{2} \xi(x, t)\right\}+\psi_{0}(x, t) \\
\xi(x, 0) & =0 .
\end{aligned}
$$

Consider now the terminal value problem for the backwards in time parabolic PDE:

$$
\begin{aligned}
\frac{\partial u(x, t)}{\partial t} & =\frac{1}{2} \nabla^{*} V^{\prime \prime}(\nabla \phi(x, t)) \nabla u(x, t), t<T, \\
u(x, T) & =u_{0}(x),
\end{aligned}
$$

with solution

$$
u(x, t)=\sum_{y \in Q} G(x, y, t, T, \phi(\cdot, \cdot)) u_{0}(y), \quad t<T .
$$

Then the solution to (6.13) is given by the formula

$$
\xi(y, T)=\int_{0}^{T} e^{-m^{2}(T-t) / 2} d t \sum_{x \in Q} G(x, y, t, T, \phi(\cdot, \cdot)) \psi_{0}(x, t), \quad y \in Q .
$$


We conclude from (6.16) that

$$
\begin{aligned}
(f(\cdot), & \left.D_{\mathrm{Mal}} \phi(x, t ; \cdot, T)\right) \\
\quad & =e^{-m^{2}(T-t) / 2}(f(\cdot), G(x, \cdot, t, T, \phi(\cdot, \cdot))), \quad x \in Q, t<T, f: Q \rightarrow \mathbf{R} .
\end{aligned}
$$

Suppose now that $F: \mathbf{R}^{L^{d}} \rightarrow \mathbf{R}$ is a $C^{1}$ function such that $|D F(z)| \leq C \exp [A|z|]$, $z \in \mathbf{R}^{L^{d}}$, for some constants $C, A$. Then from (6.17) it follows that

$$
\begin{aligned}
& D_{\mathrm{Mal}} F(x, t ; \phi(\cdot, T))=e^{-m^{2}(T-t) / 2}(d F(\cdot, \phi(\cdot, T)), G(x, \cdot, t, T, \phi(\cdot, \cdot))), \quad x \in Q, t<T, \\
& D_{\mathrm{Mal}} F(x, t ; \phi(\cdot, T))=0, \quad x \in Q, t>T .
\end{aligned}
$$

Next we observe from (6.14), (6.18) that the conditional expectation (6.10) is given by the formula

$$
\left\langle D_{\mathrm{Mal}} F(\cdot, t ; \phi(\cdot, T)) \mid \mathcal{F}_{t}\right\rangle_{\Omega_{Q, \mathrm{Mal}}}=e^{-H(T-t) / 2} d F(\cdot, \phi(\cdot, t)), \quad t<T,
$$

where the operator $H$ is as in (6.7), so $H=d^{*} d+\nabla^{*} V^{\prime \prime}(\nabla \phi(\cdot)) \nabla+m^{2}$. Since for any fixed $s \geq 0$ the distribution of $\phi(\cdot, T-s)$ converges as $T \rightarrow \infty$ to the distribution of $\phi(\cdot)$ for the invariant measure (6.1), it follows that

$$
\begin{aligned}
\lim _{T \rightarrow \infty} & \left\langle\left|\left\langle D_{\mathrm{Mal}} F(\cdot, T-s ; \phi(\cdot, T)) \mid \mathcal{F}_{T-s}\right\rangle_{\Omega_{Q, \mathrm{Mal}}}\right|^{2}\right\rangle_{\Omega_{Q, \mathrm{Mal}}} \\
& =\left\langle d F(\cdot, \phi(\cdot)) e^{-H s} d F(\cdot, \phi(\cdot))\right\rangle_{\Omega_{Q}} .
\end{aligned}
$$

Now (6.7) for $F_{1}=F_{2}$ follows from (6.9), (6.20) on letting $T \rightarrow \infty$. The identity (6.7) for general $F_{1}, F_{2}$ is then a consequence of the symmetry of the LHS of (6.7) in $F_{1}, F_{2}$.

Proposition 6.2. Let $(\Omega, \mathcal{F}, P)$ be the massive field theory probability space defined by Proposition 6.1. Then the operators $\tau_{\mathbf{e}_{j}, 0}, 1 \leq j \leq d$, on $\Omega$ are strong mixing.

Proof. We proceed as in the proof of Proposition 5.2 of [10. It will be sufficient to prove that for $k \geq 1$ and $\left(x_{j}, t_{j}\right) \in \mathbf{Z}^{d} \times \mathbf{R}, j=1, \ldots, k$,

$$
\begin{array}{r}
\lim _{n \rightarrow \infty}\left\langle f\left(\phi\left(x_{1}+n \mathbf{e}_{1}, t_{1}\right), \ldots, \phi\left(x_{k}+n \mathbf{e}_{1}, t_{k}\right)\right) g\left(\phi\left(x_{1}, t_{1}\right), \ldots, \phi\left(x_{k}, t_{k}\right)\right)\right\rangle \\
=\left\langle f\left(\phi\left(x_{1}, t_{1}\right), \ldots, \phi\left(x_{k}, t_{k}\right)\right)\right\rangle\left\langle g\left(\phi\left(x_{1}, t_{1}\right), \ldots, \phi\left(x_{k}, t_{k}\right)\right)\right\rangle
\end{array}
$$

for all $C^{\infty}$ functions $f, g: \mathbf{R}^{k} \rightarrow \mathbf{R}$ with compact support. Let $Q \subset \mathbf{Z}^{d}$ be a large cube centered at the origin with side of length an even integer $L$. We define $h_{Q, T}(n)$ for $n \in \mathbf{Z}$ and $T>0$ large by

$$
\begin{aligned}
h_{Q, T}(n)=\left\langle f\left(\phi\left(x_{1}+n \mathbf{e}_{1}, t_{1}+T\right), \ldots, \phi\left(x_{k}+n \mathbf{e}_{1}, t_{k}+T\right)\right)\right. & \\
& \left.\times g\left(\phi\left(x_{1}, t_{1}+T\right), \ldots, \phi\left(x_{k}, t_{k}+T\right)\right)\right\rangle_{\Omega_{Q}, \text { Mal }} \\
-\left\langle f\left(\phi\left(x_{1}, t_{1}+T\right), \ldots, \phi\left(x_{k}, t_{k}+T\right)\right)\right\rangle_{\Omega_{Q}, \text { Mal }} & \times\left\langle g\left(\phi\left(x_{1}, t_{1}+T\right), \ldots, \phi\left(x_{k}, t_{k}+T\right)\right)\right\rangle_{\Omega_{Q}, \text { Mal }} .
\end{aligned}
$$

The function $h_{Q, T}: \mathbf{Z} \rightarrow \mathbf{R}$ is periodic on the interval $I_{L}=\mathbf{Z} \cap[-L / 2, L / 2]$. We shall show that there is a constant $C$ independent of $L, T$ as $L, T \rightarrow \infty$ such that

$$
\sum_{n \in I_{L}}\left|h_{Q, T}(n)\right|^{2} \leq C
$$

Then (6.21) follows from (6.23) and Proposition 6.1 as in Proposition 5.2 of [10]. 
To estimate the LHS of (6.23) we go into Fourier variables, using the Plancherel theorem

$$
\sum_{n \in I_{L}}\left|h_{Q, T}(n)\right|^{2}=\frac{1}{2 \pi} \int_{\hat{I}_{L}}\left|\hat{h}_{Q, T}(\zeta)\right|^{2} d \zeta
$$

Let $a(f, \zeta, \phi(\cdot, \cdot))$ be the function

$$
a(f, \zeta, \phi(\cdot, \cdot))=\sum_{n \in I_{L}} f\left(\phi\left(x_{1}+n \mathbf{e}_{1}, t_{1}+T\right), \ldots, \phi\left(x_{k}+n \mathbf{e}_{1}, t_{k}+T\right)\right) e^{i n \zeta}
$$

Then the Fourier transform of $h_{Q, T}(\cdot)$ is bounded by

$$
\left|\hat{h}_{Q, T}(\zeta)\right|^{2} \leq \frac{1}{L^{2}} \operatorname{var}_{\Omega_{Q, \mathrm{Mal}}}[a(f, \zeta, \phi(\cdot, \cdot))] \operatorname{var}_{\Omega_{Q, \mathrm{Mal}}}[a(g, \zeta, \phi(\cdot, \cdot))] .
$$

From (6.17) we see that

$$
\begin{aligned}
& \left|D_{\mathrm{Mal}} a(x, t ; f, \zeta, \phi(\cdot, \cdot))\right| \\
& \quad \leq\|D f(\cdot)\|_{\infty} \sum_{j=1}^{k} \sum_{n \in I_{L}} e^{-m^{2}\left(T+t_{j}-t\right) / 2} G\left(x, x_{j}+n \mathbf{e}_{1}, t, T+t_{j}, \phi(\cdot, \cdot)\right)
\end{aligned}
$$

where we are using the convention $G(\cdot, \cdot, s, S)=0$ if $s>S$. It follows from (6.27) that

$$
\begin{aligned}
& \sum_{x \in Q}\left|D_{\mathrm{Mal}} a(x, t ; f, \zeta, \phi(\cdot, \cdot))\right|^{2} \\
& \leq k L\|D f(\cdot)\|_{\infty}^{2} \sum_{j=1}^{k} e^{-m^{2}\left(T+t_{j}-t\right)} \sup _{y \in Q} \sum_{x \in Q} \sum_{n \in I_{L}} G\left(x, y+n \mathbf{e}_{1}, t, T+t_{j},\right. \\
& \quad \times \phi(\cdot, \cdot)) G\left(x, y, t, T+t_{j}, \phi(\cdot, \cdot)\right) .
\end{aligned}
$$

Observe now that

$$
\sum_{y^{\prime} \in Q} G\left(x, y^{\prime}, t, T, \phi(\cdot, \cdot)\right)=\sum_{x^{\prime} \in Q} G\left(x^{\prime}, y, t, T, \phi(\cdot, \cdot)\right)=1, \quad x, y \in Q, t<T .
$$

We conclude from (6.9), (6.28), 6.29) that

$$
\operatorname{var}_{\Omega_{Q, \mathrm{Mal}}}[a(f, \zeta, \phi(\cdot, \cdot))] \leq k^{2} L\|D f(\cdot)\|_{\infty}^{2} / m^{2} .
$$

The inequality (6.23) follows from (6.24), (6.26), (6.30).

To proceed further we need to obtain a more general Poincaré inequality than was used in Proposition 6.2. In order to do this we consider functions $F(\phi(\cdot, \cdot))$ of continuous in time fields $\phi: Q \times \mathbf{R} \rightarrow \mathbf{R}$. For $h \in L^{2}(Q \times \mathbf{R})$, which is continuous in time, we define the directional derivative of $F(\phi(\cdot, \cdot))$ in direction $h$ by

$$
d F_{h}(\phi(\cdot, \cdot))=\lim _{\varepsilon \rightarrow 0}[F(\phi(\cdot, \cdot)+\varepsilon h(\cdot, \cdot))-F(\phi(\cdot, \cdot))] / \varepsilon .
$$

For the functions $F(\phi(\cdot, \cdot))$ we shall be interested in, the directional derivative (6.31) can be written as

$$
d F_{h}(\phi(\cdot, \cdot))=\sum_{x \in Q} \int_{-\infty}^{\infty} d t d F(x, t ; \phi(\cdot, \cdot)) h(x, t)=[d F(\phi(\cdot, \cdot)), h] .
$$


We shall call $d F(\cdot, \cdot ; \phi(\cdot, \cdot))$ the field derivative of $F(\phi(\cdot, \cdot))$. One can use the HS formula (6.7) to obtain a Poincaré inequality for functions $F(\phi(\cdot, \cdot))$ of the form

$$
F(\phi(\cdot, \cdot))=\int_{-\infty}^{\infty} g(t) G(\phi(\cdot, t)) d t
$$

where $g: \mathbf{R} \rightarrow \mathbf{C}$ is a continuous function of compact support and $G(\phi(\cdot))$ is a complex valued $C^{1}$ function of fields $\phi: Q \rightarrow \mathbf{R}$ which satisfies $|G(z)|+|D G(z)| \leq$ $A \exp [B|z|], z \in \mathbf{R}^{L^{d}}$, for some constants $A, B$. Evidently from (6.32) we see that the field derivative of the function (6.33) is given by the formula

$$
d F(x, t ; \phi(\cdot, \cdot))=g(t) d G(x, \phi(\cdot, t)), \quad x \in Q, t \in \mathbf{R} .
$$

Let us now define the correlation function $h: \mathbf{R} \rightarrow \mathbf{C}$ by

$$
h(t)=\langle\overline{G(\phi(\cdot, t))} G(\phi(\cdot, 0))\rangle_{\Omega_{Q}}-\langle\overline{G(\phi(\cdot, t))}\rangle_{\Omega_{Q}}\langle G(\phi(\cdot, 0))\rangle_{\Omega_{Q}} .
$$

Then the variance of $F(\phi(\cdot, \cdot))$ is given in terms of the Fourier transforms of $g(\cdot)$ and $h(\cdot)$ by

$$
\operatorname{var}_{\Omega_{Q}}[F(\phi(\cdot, \cdot))]=\frac{1}{2 \pi} \int_{-\infty}^{\infty}|\hat{g}(\zeta)|^{2} \hat{h}(\zeta) d \zeta
$$

Note that the function $\hat{h}(\cdot)$ is real and non-negative. Observe next that $h(t)$ can be written as an expectation with respect to the measure (6.1) by using the operator $d^{*} d$ which occurs in (6.7). Thus we have that

$h(t)=\left\langle e^{-d^{*} d t / 2}\left[\bar{G}(\phi(\cdot, 0))-\langle\bar{G}(\phi(\cdot, 0))\rangle_{\Omega_{Q}}\right]\left[G(\phi(\cdot, 0))-\langle G(\phi(\cdot, 0))\rangle_{\Omega_{Q}}\right]\right\rangle_{\Omega_{Q}}, \quad t>0$,

with a similar formula for $t<0$. For $\zeta \in \mathbf{R}$ let $u(\zeta, \phi(\cdot))$ be the solution to the elliptic PDE:

$$
\left[d^{*} d / 2+i \zeta\right] u(\zeta, \phi(\cdot))=\left[G(\phi(\cdot))-\langle G(\phi(\cdot))\rangle_{\Omega_{Q}}\right], \quad \phi: Q \rightarrow \mathbf{R} .
$$

We conclude from (6.37), 6.38) that

$$
\hat{h}(\zeta)=\left\langle\left[\bar{G}(\phi(\cdot, 0))-\langle\bar{G}(\phi(\cdot, 0))\rangle_{\Omega_{Q}}\right][u(\zeta, \phi(\cdot, 0))+u(-\zeta, \phi(\cdot, 0))]\right\rangle_{\Omega_{Q}} .
$$

If we apply the gradient operator $d$ to (6.38) we obtain the equation

$$
\left[d^{*} d+2 i \zeta+\nabla^{*} V^{\prime \prime}(\nabla \phi(\cdot)) \nabla+m^{2}\right] d u(\cdot, \zeta, \phi(\cdot))=2 d G(\cdot, \phi(\cdot)), \quad \phi: Q \rightarrow \mathbf{R} .
$$

Hence (6.39), (6.40) and the HS formula (6.7) imply that

$$
\begin{aligned}
\hat{h}(\zeta)= & 4 \times \\
& \quad \text { real part of } \\
& \quad d \bar{G}(\cdot, \phi(\cdot, 0))\left[d^{*} d+\nabla^{*} V^{\prime \prime}(\nabla \phi(\cdot)) \nabla+m^{2}\right]^{-1} \\
& \left.\times\left[d^{*} d+2 i \zeta+\nabla^{*} V^{\prime \prime}(\nabla \phi(\cdot)) \nabla+m^{2}\right]^{-1} d G(\cdot, \phi(\cdot, 0))\right\rangle_{\Omega_{Q}} .
\end{aligned}
$$

Just as (6.6) follows from (6.7), we see from (6.41) that

$$
0 \leq \hat{h}(\zeta) \leq \frac{4}{m^{4}}\left\langle\|d G(\phi(\cdot, 0))\|^{2}\right\rangle_{\Omega_{Q}} .
$$

It follows from (6.36), (6.42) that

$$
\operatorname{var}_{\Omega_{Q}}[F(\phi(\cdot, \cdot))] \leq \frac{4}{m^{4}}\left\langle\|d G(\cdot, \phi(\cdot, 0))\|^{2}\right\rangle_{\Omega_{Q}} \int_{-\infty}^{\infty}|g(t)|^{2} d t .
$$


Since from (6.34) the inequality (6.43) can be rewritten as

$$
\operatorname{var}_{\Omega_{Q}}[F(\phi(\cdot, \cdot))] \leq \frac{4}{m^{4}}\left\langle\|d F(\cdot, \cdot ; \phi(\cdot, \cdot))\|^{2}\right\rangle_{\Omega_{Q}},
$$

we have obtained a Poincaré inequality for functions $F(\phi(\cdot, \cdot))$ of time dependent fields which are of the form (6.33). We generalize this as follows:

Lemma 6.1. Let $F(\phi(\cdot, \cdot))$ be a bounded function of continuous in time fields $\phi$ : $Q \times \mathbf{R} \rightarrow \mathbf{R}$ which is $C^{1}$ with respect to the $L^{2}(Q \times \mathbf{R})$ metric, and assume that the field derivative function $d F(\cdot, \cdot, \phi(\cdot, \cdot))$ with range $L^{2}(Q \times \mathbf{R})$ is also bounded. Then the inequality (6.44) holds.

Proof. Let $T>0$ be large and consider $F\left(\tau_{0, T} \phi(\cdot, \cdot)\right)$ as a function of solutions $\phi(x, t), x \in Q, t>0$, to the stochastic equation (6.3). By the chain rule we have that

$$
\begin{aligned}
& D_{\mathrm{Mal}} F\left(x, t ; \tau_{0, T} \phi(\cdot, \cdot)\right) \\
& =\sum_{y \in Q} \int_{t-T}^{\infty} d s d F\left(y, s ; \tau_{0, T} \phi(\cdot, \cdot)\right) D_{\mathrm{Mal}} \phi(x, t ; y, T+s), \quad x \in Q, t>0 .
\end{aligned}
$$

It follows then from (6.17) that

$$
\begin{aligned}
& D_{\mathrm{Mal}} F\left(x, t ; \tau_{0, T} \phi(\cdot, \cdot)\right) \\
& \quad=\sum_{y \in Q} \int_{t-T}^{\infty} d s d F\left(y, s ; \tau_{0, T} \phi(\cdot, \cdot)\right) e^{-m^{2}(T+s-t) / 2} \\
& \quad \times G(x, y, t, T+s, \phi(\cdot, \cdot)), \quad x \in Q, t>0 .
\end{aligned}
$$

Hence we have that

$$
\begin{aligned}
& \quad \sum_{x \in Q} \int_{0}^{\infty} d t\left|D_{\mathrm{Mal}} F\left(x, t ; \tau_{0, T} \phi(\cdot, \cdot)\right)\right|^{2} \\
& =2 \sum_{x \in Q} \int_{0<t<T+s<T+s^{\prime}} d t d s d s^{\prime} e^{-m^{2}(T+s-t) / 2} e^{-m^{2}\left(T+s^{\prime}-t\right) / 2} h(x, s) \overline{h\left(x, s^{\prime}\right)},
\end{aligned}
$$

where

$$
h(x, s)=\sum_{y \in Q} G(x, y, t, T+s, \phi(\cdot, \cdot)) d F\left(y, s ; \tau_{0, T} \phi(\cdot, \cdot)\right) .
$$

It follows from (6.29) that

$$
\sum_{x \in Q}|h(x, s)|^{2} \leq \sum_{y \in Q}\left|d F\left(y, s ; \tau_{0, T} \phi(\cdot, \cdot)\right)\right|^{2},
$$

and so we conclude from (6.47) that

$$
\sum_{x \in Q} \int_{0}^{\infty} d t\left|D_{\mathrm{Mal}} F\left(x, t ; \tau_{0, T} \phi(\cdot, \cdot)\right)\right|^{2} \leq \frac{4}{m^{4}} \sum_{y \in Q} \int_{-\infty}^{\infty} d s\left|d F\left(y, s ; \tau_{0, T} \phi(\cdot, \cdot)\right)\right|^{2} .
$$

Hence (6.9), 6.10) imply that

$$
\operatorname{var}_{\Omega_{Q}, \text { Mal }}\left[F\left(\tau_{0, T} \phi(\cdot, \cdot)\right)\right] \leq \frac{4}{m^{4}}\left\langle\left\|d F\left(\cdot, \cdot ; \tau_{0, T} \phi(\cdot, \cdot)\right)\right\|^{2}\right\rangle_{\Omega_{Q}, \text { Mal }} .
$$


The result follows now by observing that the limit of the LHS of (6.51) as $T \rightarrow \infty$ is equal to the LHS of (6.44). Similarly the RHS of (6.51) converges to the RHS of (6.44).

We shall show how the Poincaré inequality (6.44) can be used to improve the most elementary of the inequalities contained in $\S 2$. Thus let us consider an equation which differs from (2.43) only in that the projection operator $P$ has been omitted: (6.52)

$\eta \Phi(\xi, \eta, \omega)+\partial \Phi(\xi, \eta, \omega)+\partial_{\xi}^{*} \mathbf{a}(\omega) \partial_{\xi} \Phi(\xi, \eta, \omega)=-\partial_{\xi}^{*} \mathbf{a}(\omega), \quad \eta>0, \xi \in \mathbf{R}^{d}, \omega \in \Omega$.

For any $v \in \mathbf{C}^{d}$ we multiply the row vector (6.52) on the right by the column vector $v$ and by the function $\overline{\Phi(\xi, \eta, \omega) v}$ on the left. Taking the expectation we see that

$$
\left\|P \partial_{\xi} \Phi(\xi, \eta, \cdot) v\right\| \leq\left\|\partial_{\xi} \Phi(\xi, \eta, \cdot) v\right\| \leq \frac{\Lambda|v|}{\lambda},
$$

where $\|\cdot\|$ denotes the norm in $\mathcal{H}(\Omega)$. Let $g: \mathbf{Z}^{d} \times \mathbf{R} \rightarrow \mathbf{C}^{d} \otimes \mathbf{C}^{d}$ be in $L^{p}\left(\mathbf{Z}^{d} \times \mathbf{R}, \mathbf{C}^{d} \otimes \mathbf{C}^{d}\right)$ with norm given by (3.9). If $p=1$, then (6.53) implies that

$$
\left\|P \sum_{x \in \mathbf{Z}^{d}} \int_{-\infty}^{\infty} d t g(x, t) \partial_{\xi} \Phi\left(\xi, \eta, \tau_{x,-t}\right) v\right\| \leq \frac{\Lambda|v|}{\lambda}\|g\|_{1} .
$$

The Poincaré inequality (6.44) enables us to improve (6.54) to allow $g \in$ $L^{p}\left(\mathbf{Z}^{d} \times \mathbf{R}, \mathbf{C}^{d} \otimes \mathbf{C}^{d}\right)$ for some $p>1$.

Proposition 6.3. Suppose $\mathbf{a}(\cdot)$ in (6.52) is as in the statement of Theorem 1.2. Then for $\xi \in \mathbf{R}^{d}$, $\eta>0$, there exists $p_{0}(\Lambda / \lambda)$ depending only on $d$ and $\Lambda / \lambda$ and satisfying $1<p_{0}(\Lambda / \lambda)<2$ such that for $g \in L^{p}\left(\mathbf{Z}^{d} \times \mathbf{R}, \mathbf{C}^{d} \otimes \mathbf{C}^{d}\right)$ with $1 \leq p \leq p_{0}(\Lambda / \lambda)$ and $v \in \mathbf{C}^{d}$,

$$
\left\|P \sum_{x \in \mathbf{Z}^{d}} \int_{-\infty}^{\infty} d t g(x, t) \partial_{\xi} \Phi\left(\xi, \eta, \tau_{x,-t} \cdot\right) v\right\| \leq \frac{C \Lambda_{1}|v|}{m^{2} \Lambda^{3 / 2-1 / p}}\|g\|_{p},
$$

where $\Lambda_{1}$ is the constant in Theorem 1.2 and $C$ depends only on $d$ and $\Lambda / \lambda$.

Proof. We shall first assume that $g(\cdot, \cdot)$ is continuous in time and has compact support in $\mathbf{Z}^{d} \times \mathbf{R}$. For a cube $Q$ such that $Q \times \mathbf{R}$ contains the support of $g(\cdot, \cdot)$, let $\Phi_{Q}(\xi, \eta, \cdot)$ be the solution to (6.52) with $\mathbf{a}(\phi)=\tilde{\mathbf{a}}(\phi(0,0)), \phi \in \Omega_{Q}$, so the random environment for (6.52) is $\left(\Omega_{Q}, \mathcal{F}_{Q}, P_{Q}\right)$. The inequality (6.44) implies that

$$
\begin{aligned}
& \left\|P \sum_{x \in \mathbf{Z}^{d}} \int_{-\infty}^{\infty} d t g(x, t) \partial_{\xi} \Phi\left(\xi, \eta, \tau_{x,-t}\right) v\right\|^{2} \\
& \quad \leq \frac{4}{m^{4}} \sum_{z \in Q} \int_{-\infty}^{\infty} d s\left\|\frac{\partial}{\partial \phi(z, s)} \sum_{x \in \mathbf{Z}^{d}} \int_{-\infty}^{\infty} d t g(x, t) \partial_{\xi} \Phi\left(\xi, \eta, \tau_{x,-t} \cdot\right) v\right\|^{2}
\end{aligned}
$$

where we are using the notation $\partial / \partial \phi(z, s) F(\phi(\cdot, \cdot))$ to denote the value of the field derivative $d F(z, s ; \phi(\cdot, \cdot))$ defined by (6.32) of a function $F(\phi(\cdot, \cdot))$ at $(z, s)$.

Translation operators $\tau_{x, t}, x \in \mathbf{Z}^{d}, t \in \mathbf{R}$, act on functions $F_{Q}: \Omega_{Q} \rightarrow \mathbf{C}$ by $\tau_{x, t} F_{Q}(\phi(\cdot, \cdot))=F_{Q}\left(\tau_{x, t} \phi(\cdot, \cdot)\right)$. We shall also need to use translation operators $T_{x, t}, x \in \mathbf{Z}^{d}, t \in \mathbf{R}$, which act on functions $G_{Q}: Q \times \mathbf{R} \times \Omega_{Q} \rightarrow \mathbf{C}$ by $T_{x, t} G_{Q}(z, s ; \phi(\cdot, \cdot))=G_{Q}(z+x, s+t ; \phi(\cdot, \cdot))$, so $T_{x, t}$ acts on the first two variables of $G_{Q}(\cdot, \cdot ; \phi(\cdot, \cdot))$. The operators $\tau_{x, t}, x \in \mathbf{Z}^{d}, t \in \mathbf{R}$, act on the third variable of $G_{Q}(\cdot, \cdot ; \phi(\cdot, \cdot))$, and it is clear that they commute with the $T_{x, t}, x \in \mathbf{Z}^{d}, t \in \mathbf{R}$. Let 
$F_{Q}: \Omega_{Q} \rightarrow \mathbf{C}$ be a function which is $C^{1}$ with respect to the $L^{2}(Q \times \mathbf{R})$ metric as in Lemma 6.1. One easily sees from (6.31), 6.32) that

$$
d\left[\tau_{x, t} F_{Q}\right]=T_{-x,-t} \tau_{x, t} d F_{Q}, \quad x \in \mathbf{Z}^{d}, t \in \mathbf{R},
$$

whence it follows from (2.1) that

$d\left[\partial_{j, \xi} \tau_{x,-t} F_{Q}\right]=\left[e^{-i \mathbf{e}_{j} \cdot \xi} T_{-\mathbf{e}_{j}, 0} \tau_{\mathbf{e}_{j}, 0}-1\right] T_{-x, t} \tau_{x,-t} d F_{Q}, \quad 1 \leq j \leq d, x \in \mathbf{Z}^{d}, t \in \mathbf{R}$.

Hence if we define a function $G_{Q}: Q \times \mathbf{R} \times \Omega_{Q} \rightarrow \mathbf{C}$ by

$$
G_{Q}(y, r ; \phi(\cdot, \cdot))=e^{-i y \cdot \xi} d F_{Q}\left(-y, r ; \tau_{y,-r} \phi(\cdot, \cdot)\right), \quad y \in Q, r \in \mathbf{R},
$$

then (6.58) implies that

$$
\begin{aligned}
& d\left[\partial_{j, \xi} \tau_{x,-t} F_{Q}\right](z,-s ; \phi(\cdot, \cdot)) \\
& \quad=e^{i(x-z) \cdot \xi} \nabla_{j} G_{Q}\left(x-z, t-s, \tau_{z,-s} \phi(\cdot, \cdot)\right), \quad 1 \leq j \leq d, x, z \in \mathbf{Z}^{d}, t, s \in \mathbf{R} .
\end{aligned}
$$

On taking $F_{Q}(\phi(\cdot, \cdot))=\Phi_{Q}(\xi, \eta, \phi(\cdot, \cdot)) v$ and defining $G_{Q}$ by (6.59), we conclude from (6.60) that 6.56) is the same as

$$
\begin{aligned}
& \text { 61) }\left\|P \sum_{x \in \mathbf{Z}^{d}} \int_{-\infty}^{\infty} d t g(x, t) \partial_{\xi} \Phi_{Q}\left(\xi, \eta, \tau_{x,-t} \cdot\right) v\right\|^{2} \\
& \leq \frac{4}{m^{4}} \sum_{z \in Q} \int_{-\infty}^{\infty} d s\left\|\sum_{x \in \mathbf{Z}^{d}} \int_{-\infty}^{\infty} d t g(x, t) e^{i(x-z) \cdot \xi} \nabla G_{Q}(x-z, t-s, \phi(\cdot, \cdot))\right\|^{2} .
\end{aligned}
$$

We can find an equation for $G_{Q}(\cdot, \cdot ; \phi(\cdot, \cdot))$ by applying the operator $\partial / \partial \phi(\cdot, \cdot)$ to (6.52). To see this let $h \in L^{2}(Q \times \mathbf{R})$ be $C^{1}$ as a function of time and of compact support. Then (6.52) holds for $\omega=\phi(\cdot, \cdot)$ and $\omega=\phi(\cdot, \cdot)+\varepsilon h(\cdot, \cdot)$. On subtracting the equations (6.52) for the different values of $\omega$, dividing by $\varepsilon$ and letting $\varepsilon \rightarrow 0$, we have from (6.31), 6.32) that the first term on the LHS of (6.52) converges to $\eta[d \Phi(\xi, \eta, \phi(\cdot, \cdot)) v, h]=\eta\left[d F_{Q}(\phi(\cdot, \cdot)), h\right]$. To find a similar expression for the limit as $\varepsilon \rightarrow 0$ of the second term on the LHS of (6.52), we observe that for $\delta>0$,

$$
\begin{aligned}
& \lim _{\varepsilon \rightarrow 0} \frac{F_{Q}\left(\tau_{0, \delta}[\phi(\cdot, \cdot)+\varepsilon h(\cdot, \cdot)]\right)-F_{Q}\left(\tau_{0, \delta} \phi(\cdot, \cdot)\right)}{\varepsilon} \\
&=\left[d F_{Q}\left(\tau_{0, \delta} \phi(\cdot, \cdot)\right), T_{0, \delta} h\right]=\left[T_{0,-\delta} d F_{Q}\left(\tau_{0, \delta} \phi(\cdot, \cdot)\right), h\right] .
\end{aligned}
$$

Hence, assuming one can interchange the limits $\varepsilon \rightarrow 0$ and $\delta \rightarrow 0$, we see from (6.62) that the second term on the LHS of the difference of the two equations (6.52) converges to

$$
\lim _{\delta \rightarrow 0}\left[\frac{T_{0,-\delta} d F_{Q}\left(\tau_{0, \delta} \phi(\cdot, \cdot)\right)-d F_{Q}(\phi(\cdot, \cdot))}{\delta}, h\right]=\left[D_{0} d F_{Q}(\phi(\cdot, \cdot)), h\right] .
$$

To find the limit as $\varepsilon \rightarrow 0$ of the term on the RHS of (6.52) we use the fact that $\mathbf{a}(\phi(\cdot, \cdot))=\tilde{\mathbf{a}}(\phi(0,0))$. Thus we obtain the expression

$$
\lim _{\varepsilon \rightarrow 0} \frac{\partial_{\xi}^{*} \mathbf{a}(\phi(\cdot, \cdot)) v-\partial_{\xi}^{*} \mathbf{a}(\phi(\cdot, \cdot)+\varepsilon h(\cdot, \cdot)) v}{\varepsilon}=-\left[D_{\xi}^{*}\{\delta(\cdot, \cdot) D \tilde{\mathbf{a}}(\phi(0,0)) v\}, h\right],
$$


where the operators $D_{\xi}=\left(D_{1, \xi}, \ldots, D_{d, \xi}\right)$ and $D_{\xi}^{*}=\left(D_{1, \xi}^{*}, \ldots, D_{d, \xi}^{*}\right)$ are given by the formulae

$$
D_{j, \xi}=\left[e^{-i \mathbf{e}_{j} \cdot \xi} T_{-\mathbf{e}_{j}, 0} \tau_{\mathbf{e}_{j}, 0}-1\right], \quad D_{j, \xi}^{*}=\left[e^{i \mathbf{e}_{j} \cdot \xi} T_{\mathbf{e}_{j}, 0} \tau_{-\mathbf{e}_{j}, 0}-1\right], \quad 1 \leq j \leq d .
$$

The function $\delta: Q \times \mathbf{R} \rightarrow \mathbf{R}$ in (6.64) is the delta function $\delta(0, t)=\delta(t), \delta(z, t)=$ $0, z \neq 0$, where $\delta(\cdot)$ is the Dirac delta function. The limit as $\varepsilon \rightarrow 0$ of the third term on the LHS of (6.52) can be expressed by a similar formula. Thus we have

$$
\begin{aligned}
\lim _{\varepsilon \rightarrow 0} \frac{\partial_{\xi}^{*} \mathbf{a}(\phi(\cdot, \cdot)+\varepsilon h(\cdot, \cdot)) \partial_{\xi} \Phi(\xi, \eta, \phi(\cdot, \cdot)+\varepsilon h(\cdot, \cdot)) v-\partial_{\xi}^{*} \mathbf{a}(\phi(\cdot, \cdot)) \partial_{\xi} \Phi(\xi, \eta, \phi(\cdot, \cdot)) v}{\varepsilon} \\
=\left[D_{\xi}^{*} \tilde{\mathbf{a}}(\phi(0,0)) D_{\xi} d F_{Q}(\phi(\cdot, \cdot))+D_{\xi}^{*}\left\{\delta(\cdot, \cdot) D \tilde{\mathbf{a}}(\phi(0,0)) \partial_{\xi} F_{Q}(\phi(\cdot, \cdot))\right\}, h\right] .
\end{aligned}
$$

It follows from (6.52) and (6.63)-(6.66) that $d F_{Q}(\phi(\cdot, \cdot))$ satisfies the equation

$$
\begin{aligned}
\eta d F_{Q}(\phi(\cdot, \cdot))+D_{0} d F_{Q}(\phi(\cdot, \cdot))+D_{\xi}^{*} \tilde{\mathbf{a}}(\phi(0,0)) D_{\xi} d F_{Q}(\phi(\cdot, \cdot)) \\
=-D_{\xi}^{*}\left[\delta(\cdot, \cdot) D \tilde{\mathbf{a}}(\phi(0,0))\left\{v+\partial_{\xi} F_{Q}(\phi(\cdot, \cdot))\right\}\right] .
\end{aligned}
$$

Evidently for any $(y,-r) \in \mathbf{Z}^{d} \times \mathbf{R}$ we can replace $\phi(\cdot, \cdot)$ in (6.67) by $\tau_{y,-r} \phi(\cdot, \cdot)$. If we now evaluate (6.67) with $\tau_{y,-r} \phi(\cdot, \cdot)$ substituted for $\phi(\cdot, \cdot)$ and with the first variable of $d F_{Q}\left(\cdot, \cdot ; \tau_{y,-r} \phi(\cdot, \cdot)\right)$ equal to $-y$ and the second variable equal to $r$, we obtain an equation for the function $G_{Q}(\cdot, \cdot ; \phi(\cdot, \cdot))$ of (6.59),

$$
\begin{array}{r}
\eta G_{Q}(y, r ; \phi(\cdot, \cdot))-\frac{\partial G_{Q}(y, r ; \phi(\cdot, \cdot))}{\partial r}+\nabla_{y}^{*} \tilde{\mathbf{a}}(\phi(y,-r)) \nabla_{y} G_{Q}(y, r ; \phi(\cdot, \cdot)) \\
=-\nabla_{y}^{*}\left[e^{-i y \cdot \xi} \delta(-y, r) D \tilde{\mathbf{a}}(\phi(y,-r))\left\{v+\partial_{\xi} F_{Q}\left(\tau_{y,-r} \phi(\cdot, \cdot)\right)\right\}\right] .
\end{array}
$$

We define an operator $T_{\eta}$ on functions $g: \mathbf{Z}^{d} \times \mathbf{R} \times \Omega \rightarrow \mathbf{C}^{d}$ as follows: Let $u(y, r ; \phi(\cdot, \cdot))$ be the solution to the equation

$$
\eta u(y, r ; \phi(\cdot, \cdot))-\frac{\partial u(y, r ; \phi(\cdot, \cdot))}{\partial r}+\Lambda \nabla_{y}^{*} \nabla_{y} u(y, r ; \phi(\cdot, \cdot))=\Lambda \nabla_{y}^{*} g(y, r ; \phi(\cdot, \cdot)) .
$$

Then $T_{\eta} g(y, r ; \phi(\cdot, \cdot))=\nabla_{y} u(y, r ; \phi(\cdot, \cdot)), y \in \mathbf{Z}^{d}, r \in \mathbf{R}$. It is easy to see that $T_{\eta}$ is a bounded operator on $L^{2}\left(\mathbf{Z}^{d} \times \mathbf{R} \times \Omega, \mathbf{C}^{d}\right)$ with norm $\left\|T_{\eta}\right\|$ satisfying $\left\|T_{\eta}\right\| \leq 1$. We can obtain a formula for $T_{\eta}$ which is similar to (2.47). Thus we have that

$$
T_{\eta} g(y, r ; \phi(\cdot, \cdot))=\Lambda \int_{0}^{\infty} e^{-\eta t} d t \sum_{x \in \mathbf{Z}^{d}}\left\{\nabla \nabla^{*} G_{\Lambda}(x, t)\right\} g(y-x, r+t ; \phi(\cdot, \cdot))
$$

with $G_{\Lambda}(x, t)=G(x, \Lambda t), x \in \mathbf{Z}^{d}, t>0$, and $G(\cdot, \cdot)$, the Green's function (2.46). We can similarly define operators $T_{\eta, Q}$ on periodic functions $g_{Q}: Q \times \mathbf{R} \times \Omega \rightarrow \mathbf{C}^{d}$ by extending $g_{Q}$ periodically to the function $g_{Q}: \mathbf{Z}^{d} \times \mathbf{R} \times \Omega \rightarrow \mathbf{C}^{d}$ and setting $T_{\eta, Q} g_{Q}=$ $T_{\eta} g_{Q}$. If we now take $g_{Q}$ to be given by the RHS of (6.68) so $\Lambda g_{Q}(y, r ; \phi(\cdot, \cdot))=$ $e^{-i y \cdot \xi} \delta(-y, r) D \tilde{\mathbf{a}}(\phi(y,-r))\left\{v+\partial_{\xi} F_{Q}\left(\tau_{y,-r} \phi(\cdot, \cdot)\right)\right\}$, then $T_{\eta, Q} g_{Q}(y, r ; \phi(\cdot, \cdot))=$ $e^{r \eta} h_{Q}(y, r ; \phi(\cdot, \cdot))$ where

$$
\begin{aligned}
h_{Q}(y, r ; \phi(\cdot, \cdot))= & \sum_{n \in \mathbf{Z}^{d}} e^{-i(y+n L) \cdot \xi}\left\{\nabla \nabla^{*} G_{\Lambda}(y+n L,-r)\right\} \\
& \times D \tilde{\mathbf{a}}(\phi(0,0))\left\{v+\partial_{\xi} F_{Q}(\phi(\cdot, \cdot))\right\} \quad \text { if } y \in Q, r<0, \\
h_{Q}(y, r, \phi(\cdot, \cdot))= & 0 \text { if } y \in Q, r>0,
\end{aligned}
$$

where $L$ is the length of the side of $Q$. 
We can rewrite (6.68) using the function $h_{Q}$ of (6.71). Thus let $u_{Q}(y, r ; \phi(\cdot, \cdot))$, $y \in Q, r \in \mathbf{R}$, be the solution to the periodic equation (6.69) with $g=g_{Q}$. Then (6.68), (6.69) imply that $v_{Q}=G_{Q}+u_{Q}$ is the solution to the equation

$$
\begin{aligned}
\eta v_{Q}(y, r ; \phi(\cdot, \cdot))-\frac{\partial v_{Q}(y, r ; \phi(\cdot, \cdot))}{\partial r} & +\nabla_{y}^{*} \tilde{\mathbf{a}}(\phi(y,-r)) \nabla_{y} v_{Q}(y, r ; \phi(\cdot, \cdot)) \\
= & -e^{r \eta} \Lambda \nabla_{y}^{*}\left[\tilde{\mathbf{b}}(\phi(y,-r)) h_{Q}(y, r ; \phi(\cdot, \cdot))\right]
\end{aligned}
$$

where $\tilde{\mathbf{a}}(\cdot)=\Lambda\left[I_{d}-\tilde{\mathbf{b}}(\cdot)\right]$. It follows from (6.53) that $\partial_{\xi} F_{Q}(\phi(\cdot, \cdot))$ is in $\mathcal{H}(\Omega)$ and $\left\|\partial_{\xi} F_{Q}(\phi(\cdot, \cdot))\right\| \leq \Lambda|v| / \lambda$. Since $\left|\nabla \nabla^{*} G_{\Lambda}(x, t)\right|, x \in \mathbf{Z}^{d}, t>0$, is bounded by $1 /(\Lambda t+1)$ times the RHS of (2.12), it follows from (6.71) that $h_{Q}$ is in $L^{2}\left(Q \times \mathbf{R} \times \Omega, \mathbf{C}^{d}\right)$ and $\left\|h_{Q}\right\| \leq C \sqrt{\Lambda} \Lambda_{1}|v| / \lambda$, where $C$ is a constant depending only on $d$, and $\Lambda_{1}$ is the constant in the statement of Theorem 1.2. Since from (6.72) we see that $\left\|\nabla v_{Q}\right\| \leq \Lambda\left\|h_{Q}\right\| / \lambda$, we conclude that $\left\|\nabla G_{Q}\right\| \leq C \Lambda_{1}|v|(\Lambda / \lambda)^{2} / \sqrt{\Lambda}$. It follows now from (6.61) and Young's inequality that (6.55) holds for $p=1$ provided we can show that the LHS of (6.61) converges as $Q \rightarrow \mathbf{Z}^{d}$ to the LHS of (6.55). To see this note that we are assuming that the function $g(\cdot, \cdot)$ in (6.61) has compact support and that $\Re \eta>0$. Hence we can use the perturbation expansion obtained from (2.13) and Proposition 6.1 to prove the convergence.

We can also show that the RHS of (6.61) converges as $Q \rightarrow \mathbf{Z}^{d}$ by generating the function $\nabla G_{Q}$ from a perturbation expansion. Thus let $\mathbf{B}: \mathbf{Z}^{d} \times \mathbf{R} \times \Omega \rightarrow \mathbf{C}^{d} \otimes \mathbf{C}^{d}$ be defined by $\mathbf{B}(y, r ; \phi(\cdot, \cdot))=\tilde{\mathbf{b}}(\phi(y,-r)), y \in \mathbf{Z}^{d}, r \in \mathbf{R}$. It follows from (6.69), (6.72) that $\nabla v_{Q}$ is the solution to the equation

$$
\nabla v_{Q}(\cdot, \cdot ; \phi(\cdot, \cdot))=T_{\eta, Q}\left[\mathbf{B}(\cdot, \cdot ; \phi(\cdot, \cdot))\left\{\nabla v_{Q}(\cdot, \cdot ; \phi(\cdot, \cdot))-e^{r \eta} h_{Q}(\cdot, \cdot ; \phi(\cdot, \cdot))\right\}\right]
$$

Since $g \in L^{1}\left(\mathbf{Z}^{d} \times \mathbf{R}\right)$ it follows by the uniform in $Q$ estimates of the previous paragraph that it is sufficient to prove convergence as $Q \rightarrow \mathbf{Z}^{d}$ for any finite number of terms in the Neumann series expansion of (6.73). The convergence for a finite number of terms follows from Proposition 6.1 using the fact that the function $g(\cdot, \cdot)$ in (6.61) has compact support and that $\Re \eta>0$. We have shown now that

$$
\begin{aligned}
& \quad\left\|P \sum_{x \in \mathbf{Z}^{d}} \int_{-\infty}^{\infty} d t g(x, t) \partial_{\xi} \Phi\left(\xi, \eta, \tau_{x,-t} \cdot\right) v\right\|^{2} \\
& \leq \frac{4}{m^{4}} \sum_{z \in \mathbf{Z}^{d}} \int_{-\infty}^{\infty} d s\left\|\sum_{x \in \mathbf{Z}^{d}} \int_{-\infty}^{\infty} d t g(x, t) e^{i(x-z) \cdot \xi} \nabla G(x-z, t-s, \phi(\cdot, \cdot))\right\|^{2},
\end{aligned}
$$

where $\nabla G=\nabla v-h$ with

$$
\begin{aligned}
h(y, r ; \phi(\cdot, \cdot))= & \left\{\nabla \nabla^{*} G_{\Lambda}(y,-r)\right\}^{*} D \tilde{\mathbf{a}}(\phi(0,0))\left\{v+\partial_{\xi} F_{Q}(\phi(\cdot, \cdot))\right\} \\
& \text { if } y \in \mathbf{Z}^{d}, r<0 ; \quad h(y, r, \phi(\cdot, \cdot))=0 \text { if } y \in \mathbf{Z}^{d}, r>0,
\end{aligned}
$$

and $\nabla v$ is the solution to the equation

$$
\nabla v(\cdot, \cdot ; \phi(\cdot, \cdot))=T_{\eta}\left[\mathbf{B}(\cdot, \cdot ; \phi(\cdot, \cdot))\left\{\nabla v(\cdot, \cdot ; \phi(\cdot, \cdot))-e^{r \eta} h(\cdot, \cdot ; \phi(\cdot, \cdot))\right\}\right] .
$$

We can now easily extend the previous argument by using the continuous time version of the Calderon-Zygmund theorem, Corollary 5.1, to prove (6.55) for a range of $p>1$. Define for $q \geq 1$ the Banach space $L^{q}\left(\mathbf{Z}^{d} \times \mathbf{R} \times \Omega, \mathbf{C}^{d}\right)$ of functions 
$g: \mathbf{Z}^{d} \times \mathbf{R} \times \Omega \rightarrow \mathbf{C}^{d}$ with norm $\|g\|_{q}$ given by

$$
\|g\|_{q}^{q}=\sum_{y \in \mathbf{Z}^{d}} \int_{-\infty}^{\infty} d t\|g(y, r ; \phi(\cdot, \cdot))\|^{q},
$$

where $\|g(y, r ; \phi(\cdot, \cdot))\|$ is the norm of $g(y, r ; \phi(\cdot, \cdot)) \in \mathcal{H}(\Omega)$. By following the argument of Lemma 5.2, we see that $T_{\eta}$ is bounded on $L^{q}\left(\mathbf{Z}^{d} \times \mathbf{R} \times \Omega, \mathbf{C}^{d}\right)$ for $q>1$ with norm $\left\|T_{\eta}\right\|_{q} \leq 1+\delta(q)$, where $\lim _{q \rightarrow 2} \delta(q)=0$. Noting that $\|h\|_{q} \leq$ $C_{q} \Lambda^{1-1 / q} \Lambda_{1}|v| / \lambda$ for a constant $C_{q}$ depending only on $d, q$, we conclude from (6.76) and the Calderon-Zygmund theorem that there exists $q_{0}(\Lambda / \lambda)<2$ depending only on $d, \Lambda / \lambda$, such that $\nabla G$ is in $L^{q}\left(\mathbf{Z}^{d} \times \mathbf{R} \times \Omega, \mathbf{C}^{d}\right)$ for $q_{0}(\Lambda / \lambda) \leq q \leq 2$ and $\|\nabla G\|_{q} \leq C \Lambda^{-1 / q} \Lambda_{1}|v|$ where the constant $C$ depends only on $d, \Lambda / \lambda$. The inequality (6.55) with $p=2 q /(3 q-2)$ follows from (6.74) and Young's inequality.

In order to establish Hypothesis 4.2 for the massive field theory environment $(\Omega, \mathcal{F}, P)$ we shall need a refinement of the Poincaré inequality (6.44). We can see what this refinement should be by considering again functions of the form (6.33), for which (6.36) and (6.41) hold. It follows from (6.41) that $\hat{h}(\zeta)$ satisfies the inequality

$$
0 \leq \hat{h}(\zeta) \leq \frac{4}{m^{4}+4 \zeta^{2}}\left\langle\|d G(\phi(\cdot, 0))\|^{2}\right\rangle_{\Omega_{Q}} .
$$

Substituting the RHS of (6.78) into (6.36) we obtain the inequality

$$
\begin{gathered}
\operatorname{var}_{\Omega_{Q}}[F(\phi(\cdot, \cdot))] \leq \frac{1}{m^{2}} \int_{-\infty}^{\infty} \int_{-\infty}^{\infty} g(t) \overline{g(s)} e^{-m^{2}|t-s| / 2} d t d s\left\langle\|d G(\phi(\cdot, 0))\|^{2}\right\rangle_{\Omega_{Q}} \\
\leq \frac{4}{m^{4}} \int_{-\infty}^{\infty}|g(t)|^{2} d t\left\langle\|d G(\phi(\cdot, 0))\|^{2}\right\rangle_{\Omega_{Q}}=\frac{4}{m^{4}}\left\langle\|d F(\cdot, \cdot ; \phi(\cdot, \cdot))\|^{2}\right\rangle_{\Omega_{Q}} .
\end{gathered}
$$

Observe now that the first integral on the RHS of (6.79) can be written as a convolution $\left[g, f^{*} g\right]$ where $f(t)=m^{-2} e^{-m^{2}|t| / 2}, t \in \mathbf{R}$. Hence it follows from Young's inequality that for $1 \leq p \leq 2$,

$$
\operatorname{var}_{\Omega_{Q}}[F(\phi(\cdot, \cdot))] \leq \frac{C}{m^{2(3-2 / p)}}\|g\|_{p}^{2}\left\langle\|d G(\phi(\cdot, 0))\|^{2}\right\rangle_{\Omega_{Q}},
$$

where $\|g\|_{p}$ denotes the $L^{p}$ norm of $g(\cdot)$ and $C$ is a universal constant. The Poincaré inequality (6.44) only implies (6.80) for $p=2$.

We shall also need a continuous time version of Corollary 5.1, as we already did in the proof of Proposition 6.3. Thus let $T_{\xi, \eta}$ act on functions $g: \mathbf{Z}^{d} \times \mathbf{R} \rightarrow \mathbf{C}^{d}$ as

$$
T_{\xi, \eta} g(y, r)=\Lambda \int_{0}^{\infty} e^{-\eta t} d t \sum_{x \in \mathbf{Z}^{d}}\left\{\nabla \nabla^{*} G_{\Lambda}(x, t)\right\} e^{i x \cdot \xi} g(y-x, r+t) .
$$

Comparing the operator $T_{\xi, \eta}$ of (6.81) to the operator $T_{\xi, \eta}$ of (5.2), we see that one can easily extend the argument of $\S 4$ to obtain a continuous time version of Corollary 5.1:

Corollary 6.1. For $(\xi, \eta)$ satisfying the assumptions of Lemma 5.2 the operator $T_{\xi, \eta}$ of (6.81) is bounded on $\mathcal{H}^{p}\left(\mathbf{Z}^{d} \times \mathbf{R}\right)$ for $3 / 2 \leq p \leq 3$, and $\left\|T_{\xi, \eta}\right\|_{p} \leq$ $[1+\delta(p)]\left(1+C_{2}|\Im \xi|^{2} /[\Re \eta / \Lambda]\right)$, where the function $\delta(\cdot)$ depends only on $d$ and $\lim _{p \rightarrow 2} \delta(p)=0$. 
Proof of Hypothesis 4.2. We shall first prove Hypothesis 3.2. We assume $g: \mathbf{Z}^{d} \times$ $\mathbf{R} \rightarrow \mathbf{C}^{d} \otimes \mathbf{C}^{d}$ has compact support, and for $k=1,2, \ldots$, we denote by $a_{k}(g, \xi, \eta)$ the random $d \times d$ matrix

$$
a_{k}(g, \xi, \eta)=\sum_{x \in \mathbf{Z}^{d}} \int_{-\infty}^{\infty} d t g(x, t) \tau_{x,-t} P \mathbf{b}(\cdot)\left[P T_{\xi, \eta} \mathbf{b}(\cdot)\right]^{k-1} .
$$

Evidently Hypothesis 3.2 will follow if we can show there is a constant $C$ such that

$$
\sum_{k=1}^{\infty}\left\|a_{k}(g, \xi, \eta) v\right\| \leq C\|g\|_{p}|v| \quad \text { for } 1 \leq p \leq p_{0}(\Lambda / \lambda), v \in \mathbf{C}^{d} .
$$

We establish (6.83) by obtaining a bound $\left\|a_{k}(g, \xi, \eta) v\right\| \leq C_{k}\|g\|_{p}|v|$ where $C_{k}$ decays exponentially in $k$ as $k \rightarrow \infty$.

In the case $k=1$ we have from the Poincaré inequality (6.44) that

$$
\begin{aligned}
\left\|a_{1}(g, \xi, \eta) v\right\|^{2} & \leq \frac{4}{m^{4}} \sum_{x \in \mathbf{Z}^{d}} \int_{-\infty}^{\infty} d t\|g(x, t) D \tilde{\mathbf{b}}(\phi(0,0)) v\|^{2} \\
& \leq\left(\frac{2 \Lambda_{1}}{m^{2} \Lambda}\|g\|_{2}|v|\right)^{2} .
\end{aligned}
$$

We also have that $a_{1}(g, \xi, \eta)=\hat{g}(A, B) P \mathbf{b}(\cdot)$, where $\hat{g}$ is the Fourier transform (2.48) of $g$ and $A, B$ are the self-adjoint operators (2.50), (2.51). Hence we have that $\left\|a_{1}(g, \xi, \eta) v\right\| \leq\|g\|_{1}|v|$. We conclude therefore from (6.84) and the Riesz-Thorin interpolation theorem [31] that there is a constant $C_{1}$ such that $\left\|a_{1}(g, \xi, \eta) v\right\| \leq$ $C_{1}\|g\|_{p}|v|$ for $1 \leq p \leq 2$.

When $k>1$ we write

$$
a_{k}(g, \xi, \eta) v=P \sum_{x \in \mathbf{Z}^{d}} \int_{-\infty}^{\infty} d t g(x, t) \tau_{x,-t} \mathbf{b}(\cdot) \partial_{\xi} F_{k}(\phi(\cdot, \cdot)),
$$

where the functions $F_{k}(\phi(\cdot, \cdot))$ are defined inductively. For $\xi \in \mathbf{R}^{d}$ the $F_{k}(\phi(\cdot, \cdot))$ satisfy the recurrence equations

$$
[\eta+\partial] F_{2}(\phi(\cdot, \cdot))+\Lambda \partial_{\xi}^{*} \partial_{\xi} F_{2}(\phi(\cdot, \cdot))=\Lambda P \partial_{\xi}^{*}[\tilde{\mathbf{b}}(\phi(0,0)) v]
$$$$
[\eta+\partial] F_{k}(\phi(\cdot, \cdot))+\Lambda \partial_{\xi}^{*} \partial_{\xi} F_{k}(\phi(\cdot, \cdot))=\Lambda P \partial_{\xi}^{*}\left[\tilde{\mathbf{b}}(\phi(0,0)) \partial_{\xi} F_{k-1}(\phi(\cdot, \cdot))\right] \text { if } k>2 \text {. }
$$

The $F_{k}(\phi(\cdot, \cdot))$ for $\xi \in \mathbf{C}^{d}$ are defined by analytic continuation from the values of $F_{k}(\phi(\cdot, \cdot))$ when $\xi \in \mathbf{R}^{d}$. Similarly to (6.59) we define for $k \geq 2$ functions $G_{k}: \mathbf{Z}^{d} \times \mathbf{R} \times \Omega \rightarrow \mathbf{C}$ by

$$
G_{k}(y, r ; \phi(\cdot, \cdot))=e^{-i y \cdot \xi} d F_{k}\left(-y, r ; \tau_{y,-r} \phi(\cdot, \cdot)\right), \quad y \in \mathbf{Z}^{d}, r \in \mathbf{R} .
$$

Then from (6.86) we see that the $G_{k}(y, r ; \phi(\cdot, \cdot))$ satisfy the equations

$$
\begin{gathered}
\eta G_{2}(y, r ; \phi(\cdot, \cdot))-\frac{\partial G_{2}(y, r ; \phi(\cdot, \cdot))}{\partial r}+\Lambda \nabla_{y}^{*} \nabla_{y} G_{2}(y, r ; \phi(\cdot, \cdot)) \\
=\Lambda P \nabla_{y}^{*}\left[e^{-i y \cdot \xi} \delta(-y, r) D \tilde{\mathbf{b}}(\phi(y,-r)) v\right] \\
\eta G_{k}(y, r ; \phi(\cdot, \cdot))-\frac{\partial G_{k}(y, r ; \phi(\cdot, \cdot))}{\partial r}+\Lambda \nabla_{y}^{*} \nabla_{y} G_{k}(y, r ; \phi(\cdot, \cdot)) \\
=\Lambda P \nabla_{y}^{*}\left[e^{-i y \cdot \xi} \delta(-y, r) D \tilde{\mathbf{b}}(\phi(y,-r)) \partial_{\xi} F_{k-1}\left(\tau_{y,-r} \phi(\cdot, \cdot)\right)\right. \\
\left.+\tilde{\mathbf{b}}(\phi(y,-r)) \nabla_{y} G_{k-1}(y, r ; \phi(\cdot, \cdot))\right] \quad \text { if } k>2 .
\end{gathered}
$$


Instead of estimating the norm of the function $a_{k}(g, \xi, \eta) v$ of (6.85) directly by using the Poincaré inequality as in (6.61), we begin with the Clark-Okone formula (6.9). Let $\phi(\cdot, t), t>0$, be the solution of (6.3) with initial condition $\phi(\cdot, 0)=0$. We extend the function $\phi(\cdot, t)$ to $t<0$ by setting $\phi(\cdot, t)=0$ for $t<0$. It is then easy to see that

$$
\left\|a_{k}(g, \xi, \eta) v\right\|^{2}=\lim _{T \rightarrow \infty} \operatorname{var}_{\Omega_{Q}, \operatorname{Mal}}\left[H\left(\tau_{0, T} \phi(\cdot, \cdot)\right)\right],
$$

where the function $H(\phi(\cdot, \cdot))$ is given by the formula

$$
H(\phi(\cdot, \cdot))=\sum_{y \in \mathbf{Z}^{d}} \int_{-\infty}^{\infty} d s g(y, s) \tilde{\mathbf{b}}(\phi(y,-s)) \partial_{\xi} F_{k}\left(\tau_{y,-s} \phi(\cdot, \cdot)\right)
$$

We have now from (6.45) that for $x \in Q, t>0$, the Malliavin derivative

$$
D_{\mathrm{Mal}} H\left(x, t ; \tau_{0, T} \phi(\cdot, \cdot)\right)=\sigma_{1, T}(x, t ; \phi(\cdot, \cdot))+\sigma_{2, T}(x, t ; \phi(\cdot, \cdot)),
$$

where

$$
\begin{aligned}
& \sigma_{1, T}(x, t ; \phi(\cdot, \cdot)) \\
& \quad=\sum_{y \in \mathbf{Z}^{d}} \int_{-\infty}^{T-t} d s g(y, s) e^{-m^{2}(T-t-s) / 2} G(x, y, t, T-s, \phi(\cdot, \cdot)) \\
& \quad \times D \tilde{\mathbf{b}}(\phi(y, T-s)) \partial_{\xi} F_{k}\left(\tau_{y, T-s} \phi(\cdot, \cdot)\right),
\end{aligned}
$$

with $G(\cdot, \cdot, \cdot, \cdot, \phi(\cdot, \cdot))$ being Green's function (6.15). The function $\sigma_{2, T}(x, t ; \phi(\cdot, \cdot))$ is given by the formula

$$
\sigma_{2, T}(\cdot, \cdot ; \phi(\cdot, \cdot))=\sum_{y \in \mathbf{Z}^{d}} \int_{-\infty}^{\infty} d s g(y, s) \tilde{\mathbf{b}}(\phi(y, T-s)) D_{\mathrm{Mal}}\left[\partial_{\xi} F_{k}\left(\tau_{y, T-s} \phi(\cdot, \cdot)\right)\right] .
$$

It follows from (6.9) that

$$
\begin{aligned}
\left\|a_{k}(g, \xi, \eta) v\right\| & \leq \lim _{T \rightarrow \infty}\left[\sum_{x \in Q} \int_{0}^{\infty} d t\left|\left\langle\sigma_{1, T}(x, t ; \phi(\cdot, \cdot)) \mid \mathcal{F}_{t}\right\rangle_{\Omega_{Q, \mathrm{Mal}}}\right|^{2}\right]^{1 / 2} \\
& +\lim _{T \rightarrow \infty}\left[\sum_{x \in Q} \int_{0}^{\infty} d t\left|\left\langle\sigma_{2, T}(x, t ; \phi(\cdot, \cdot)) \mid \mathcal{F}_{t}\right\rangle_{\Omega_{Q, \mathrm{Mal}}}\right|^{2}\right]^{1 / 2}
\end{aligned}
$$

To estimate the first term on the RHS of (6.93) we argue as in Lemma 6.1. Thus from (6.91) we have that

$$
\begin{aligned}
& \text { (6.94) } \sum_{x \in Q} \int_{0}^{\infty} d t\left|\sigma_{1, T}(x, t ; \phi(\cdot, \cdot))\right|^{2} \\
& =2 \sum_{x \in Q} \int_{0<t<T-s<T-s^{\prime}} d t d s d s^{\prime} e^{-m^{2}(T-t-s) / 2} e^{-m^{2}\left(T-t-s^{\prime}\right) / 2} h(x, s) \cdot \overline{h\left(x, s^{\prime}\right)},
\end{aligned}
$$

where

$$
h(x, s)=\sum_{y \in Q} G(x, y, t, T-s, \phi(\cdot, \cdot)) g(y, s) D \tilde{\mathbf{b}}(\phi(y, T-s)) \partial_{\xi} F_{k}\left(\tau_{y, T-s} \phi(\cdot, \cdot)\right) .
$$


It follows from (6.29) that

$$
\sum_{x \in Q}|h(x, s)|^{2} \leq \sum_{y \in Q}\left|g(y, s) D \tilde{\mathbf{b}}(\phi(y, T-s)) \partial_{\xi} F_{k}\left(\tau_{y, T-s} \phi(\cdot, \cdot)\right)\right|^{2},
$$

and so we conclude from (6.94) that

$$
\begin{aligned}
& \sum_{x \in Q} \int_{0}^{\infty} d t\left|\sigma_{1, T}(x, t ; \phi(\cdot, \cdot))\right|^{2} \\
& \quad \leq \frac{1}{m^{2}} \int_{t-T}^{\infty} \int_{t-T}^{\infty} d s d s^{\prime} e^{-m^{2}\left|s-s^{\prime}\right| / 2} k\left(s, \tau_{0, T} \phi(\cdot, \cdot)\right) k\left(s^{\prime}, \tau_{0, T} \phi(\cdot, \cdot)\right) .
\end{aligned}
$$

The function $k(s, \phi(\cdot, \cdot))$ is given by the formula

$$
\begin{aligned}
k(s, \phi(\cdot, \cdot)) & =\left[\sum_{y \in Q}\left|g(y, s) D \tilde{\mathbf{b}}(\phi(y,-s)) \partial_{\xi} F_{k}\left(\tau_{y,-s} \phi(\cdot, \cdot)\right)\right|^{2}\right]^{1 / 2} \\
& =\|g(\cdot, s)\|_{2} k_{1}(s, \phi(\cdot, \cdot)),
\end{aligned}
$$

where $\|g(\cdot, s)\|_{2}$ denotes the $L^{2}$ norm of the $d \times d$ matrix valued function $g(y, s)$, $y \in \mathbf{Z}^{d}$. Observe next that (6.97), (6.98) and the Schwarz inequality imply that

$$
\begin{aligned}
& \sum_{x \in Q} \int_{0}^{\infty} d t\left|\sigma_{1, T}(x, t ; \phi(\cdot, \cdot))\right|^{2} \\
& \quad \leq \frac{1}{m^{2}} \int_{t-T}^{\infty} \int_{t-T}^{\infty} d s d s^{\prime} e^{-m^{2}\left|s-s^{\prime}\right| / 2}\|g(\cdot, s)\|_{2}\left\|g\left(\cdot, s^{\prime}\right)\right\|_{2} k_{1}\left(s, \tau_{0, T} \phi(\cdot, \cdot)\right)^{2} .
\end{aligned}
$$

Hence on using the fact that for any $s \in \mathbf{R}$ one has

$$
\lim _{T \rightarrow \infty}\left\langle k_{1}\left(s, \tau_{0, T} \phi(\cdot, \cdot)\right)^{2}\right\rangle_{\Omega_{Q, \text { Mal }}} \leq \frac{C \Lambda_{1}^{2}}{\Lambda^{2}}\left\langle\left|\partial_{\xi} F_{k}(\phi(\cdot, \cdot))\right|^{2}\right\rangle_{\Omega_{Q}},
$$

where the constant $C$ depends only on $d$, we conclude as in the argument showing (6.80) that for $1 \leq p \leq 2$ there is a constant $C$ depending only on $d$ such that

$$
\begin{aligned}
\lim _{T \rightarrow \infty} \sum_{x \in Q} & \int_{0}^{\infty} d t\left|\left\langle\sigma_{1, T}(x, t ; \phi(\cdot, \cdot)) \mid \mathcal{F}_{t}\right\rangle_{\Omega_{Q, \mathrm{Mal}}}\right|^{2} \\
& \leq \frac{C \Lambda_{1}^{2}}{\Lambda^{2} m^{2(3-2 / p)}}\left[\int_{-\infty}^{\infty}\|g(\cdot, s)\|_{2}^{p} d s\right]^{2 / p}\left\langle\left|\partial_{\xi} F_{k}(\phi(\cdot, \cdot))\right|^{2}\right\rangle_{\Omega_{Q}} .
\end{aligned}
$$

From (6.86) we see that for $\xi \in \mathbf{R}^{d}$,

$$
\left\langle\left|\partial_{\xi} F_{k}(\phi(\cdot, \cdot))\right|^{2}\right\rangle_{\Omega_{Q}} \leq(1-\lambda / \Lambda)^{2(k-1)}|v|^{2},
$$

and so (6.101) implies that for $\xi \in \mathbf{R}^{d}$ the first term on the RHS of (6.93) is bounded as

$$
\begin{aligned}
\lim _{T \rightarrow \infty} & \sum_{x \in Q} \int_{0}^{\infty} d t\left|\left\langle\sigma_{1, T}(x, t ; \phi(\cdot, \cdot)) \mid \mathcal{F}_{t}\right\rangle_{\Omega_{Q, \mathrm{Mal}}}\right|^{2} \\
\leq & \left\{\frac{C \Lambda_{1}}{\Lambda m^{3-2 / p}}\|g(\cdot, \cdot)\|_{p}(1-\lambda / \Lambda)^{k-1}|v|\right\}^{2},
\end{aligned}
$$

where the $p$ norm of $g(\cdot, \cdot)$ is given by (3.9). 
We can estimate the second term on the RHS of (6.93) by following the argument of Proposition 6.3. Thus we have that

$$
\begin{aligned}
& \lim _{T \rightarrow \infty} \sum_{x \in Q} \int_{0}^{\infty} d t\left|\left\langle\sigma_{2, T}(x, t ; \phi(\cdot, \cdot)) \mid \mathcal{F}_{t}\right\rangle_{\Omega_{Q, \mathrm{Mal}}}\right|^{2} \\
& \leq \frac{4}{m^{4}} \sum_{z \in Q} \int_{-\infty}^{\infty} d s\langle| \sum_{x \in \mathbf{Z}^{d}} \int_{-\infty}^{\infty} d t g(x, t) \tilde{\mathbf{b}}(\phi(x,-t)) e^{i(x-z) \cdot \xi} \\
& \left.\times\left.\nabla G_{k}\left(x-z, t-s ; \tau_{z,-s} \phi(\cdot, \cdot)\right)\right|^{2}\right\rangle_{\Omega_{Q}} \\
& =\frac{4}{m^{4}} \sum_{z \in Q} \int_{-\infty}^{\infty} d s\langle| \sum_{x \in \mathbf{Z}^{d}} \int_{-\infty}^{\infty} d t g(x, t) \tilde{\mathbf{b}}(\phi(x-z, s-t)) e^{i(x-z) \cdot \xi} \\
& \times\left.\nabla G_{k}(x-z, t-s ; \phi(\cdot, \cdot))\right|_{\Omega_{Q}} ^{2}
\end{aligned}
$$

where we have used the invariance of the operators $\tau_{z,-s}, z \in \mathbf{Z}^{d}, s \in \mathbf{R}$, on $\left(\Omega_{Q}, \mathcal{F}_{Q}, P_{Q}\right)$. As in Proposition 6.3 we are justified in taking the limit $Q \rightarrow \mathbf{Z}^{d}$ in (6.104), and hence (6.69), 6.70), (6.88) imply that $\nabla G_{2}(\cdot, \cdot ; \phi(\cdot, \cdot))$ is given by the formula

$$
\begin{aligned}
& \nabla G_{2}(y, r ; \phi(\cdot, \cdot))=\Lambda e^{\eta r} \nabla \nabla^{*} G_{\Lambda}(y,-r) P[D \tilde{\mathbf{b}}(\phi(0,0)) v] \\
& \quad \text { if } y \in \mathbf{Z}^{d}, r<0 ; \quad \nabla G_{2}(y, r ; \phi(\cdot, \cdot))=0 \text { if } y \in \mathbf{Z}^{d}, r>0 .
\end{aligned}
$$

We similarly have that for $k>2$,

$$
\nabla G_{k}(y, r ; \phi(\cdot, \cdot))=e^{\eta r} h_{k}(y, r ; \phi(\cdot, \cdot))+P T_{\eta}\left[\mathbf{B}(\cdot, \cdot ; \phi(\cdot, \cdot)) \nabla G_{k-1}(\cdot, \cdot ; \phi(\cdot, \cdot))\right],
$$

where the function $\mathbf{B}(\cdot, \cdot ; \phi(\cdot, \cdot))$ is as in (6.73) and

$$
\begin{aligned}
h_{k}(y, r ; \phi(\cdot, \cdot)) & =\Lambda \nabla \nabla^{*} G_{\Lambda}(y,-r) P\left[D \tilde{\mathbf{b}}(\phi(0,0)) \partial_{\xi} F_{k-1}(\phi(\cdot, \cdot))\right] \\
& \text { if } y \in \mathbf{Z}^{d}, r<0 ; \quad h_{k}(y, r ; \phi(\cdot, \cdot))=0 \text { if } y \in \mathbf{Z}^{d}, r>0 .
\end{aligned}
$$

Define the function $A_{k}: \mathbf{Z}^{d} \times \mathbf{R} \times \Omega \rightarrow \mathbf{C}^{d}$ by $A_{k}(y, r ; \phi(\cdot, \cdot))=e^{i y \cdot \xi} \nabla G_{k}(y, r ; \phi(\cdot, \cdot))$. Then we see from (6.106) that for $k>2$ the function $A_{k}(\cdot, \cdot ; \phi(\cdot, \cdot))$ satisfies the equation

$$
A_{k}(\cdot, \cdot ; \phi(\cdot, \cdot))=D_{k}(\cdot, \cdot ; \phi(\cdot, \cdot))+P T_{\xi, \eta}\left[\mathbf{B}(\cdot, \cdot ; \phi(\cdot, \cdot)) A_{k-1}(\cdot, \cdot ; \phi(\cdot, \cdot))\right],
$$

where $D_{k}(y, r ; \phi(\cdot, \cdot))=e^{i y \cdot \xi+\eta r} h_{k}(y, r ; \phi(\cdot, \cdot))$, and from (6.70) it follows that the operator $T_{\xi, \eta}$ is given by (6.81).

Just as in Proposition 6.3 we see that if $\|\cdot\|_{q}$ denotes the $q$ norm (6.77), then for $\xi \in \mathbf{R}^{d}$ and $1<q \leq 2$,

$$
\left\|A_{2}\right\|_{q} \leq C_{q} \Lambda_{1}|v| / \Lambda^{1 / q}, \quad\left\|D_{k}\right\|_{q} \leq C_{q}(1-\lambda / \Lambda)^{k-2} \Lambda_{1}|v| / \Lambda^{1 / q} \text { for } k>2,
$$


where the constant $C_{q}$ depends only on $d, q$ and diverges as $q \rightarrow 1$. It follows then from (6.108), (6.109) that for $\xi \in \mathbf{R}^{d}$ one has the inequality $\left\|A_{k}\right\|_{2} \leq$ $C k(1-\lambda / \Lambda)^{k-2} \Lambda_{1}|v| / \Lambda^{1 / 2}$ for some constant $C$ depending only on $d$. We can extend this inequality by using Corollary 6.1. Thus for $(\xi, \eta)$ satisfying the conditions of Lemma 5.2 for sufficiently small constant $C_{1}$ depending only on $d$, there exists $q_{0}(\Lambda / \lambda)<2$ such that for some constant $C$ depending only on $d, \Lambda / \lambda$ one has the inequality

(6.110) $\left\|A_{k}\right\|_{q} \leq C k(1-\lambda / \Lambda)^{k-2}\left(1+C_{2}|\Im \xi|^{2} /[\Re \eta / \Lambda]\right)^{k-2} \Lambda_{1}|v| / \Lambda^{1 / q}$ for $k \geq 2$,

provided $q_{0}(\Lambda / \lambda) \leq q \leq 2$. We can now bound the RHS of (6.104) in terms of $\|g\|_{p}$ with $p=2 q /(3 q-2)$ by using (6.110) and Young's inequality. If we combine this with the inequality (6.103), then we conclude from (6.93) that

$$
\begin{aligned}
& \left\|a_{k}(g, \xi, \eta) v\right\| \leq \frac{C \Lambda_{1}}{\Lambda m^{3-2 / p}}\|g(\cdot, \cdot)\|_{p}(1-\lambda / \Lambda)^{k-1}|v| \\
& \quad+\frac{C k \Lambda_{1}}{m^{2} \Lambda^{3 / 2-1 / p}}\|g(\cdot, \cdot)\|_{p}(1-\lambda / \Lambda)^{k-2}\left(1+C_{2}|\Im \xi|^{2} /[\Re \eta / \Lambda]\right)^{k-2}|v| .
\end{aligned}
$$

Evidently the inequality (6.111) implies that 6.83) holds provided $(\xi, \eta)$ satisfy the conditions of Lemma 5.2 for sufficiently small $C_{1}$ depending only on $d, \Lambda / \lambda$. Restricting $\xi$ to be in $\mathbf{R}^{n}$ (3.10) then follows, and hence we have proven that Hypothesis 3.2 holds in the massive field theory case.

To complete the proof of Hypothesis 4.2 we first observe that the above argument immediately applies to the situation where the functions $g_{2}, \ldots, g_{k}$ are delta functions $g_{j}(x, t)=\delta\left(x-x_{j}, t-t_{j}\right), j=2, \ldots, k$. The inequality (4.72) then follows for general $g_{2}, \ldots, g_{k} \in L^{1}\left(\mathbf{Z}^{d} \times \mathbf{R}, \mathbf{C}^{d} \otimes \mathbf{C}^{d}\right)$ from the triangle inequality.

\section{Appendix A. Optimal Rates of COnVERGence}

Here we shall show that if $\lambda / \Lambda$ is sufficiently close to 1 , then one can take $\alpha=1$ in Theorem 1.2. First we prove that if $\lambda / \Lambda$ is sufficiently close to 1 , then some derivatives of the function $q(\xi, \eta)$ are uniformly bounded as $\eta \rightarrow 0$.

Lemma A.1. Assume $(\Omega, \mathcal{F}, P)$ and $\mathbf{a}(\cdot)$ are as in the statement of Theorem 1.2. Then there exists $\delta>0$ depending only on $d$ such that if $1-\lambda / \Lambda<\delta$ the $d \times d$ matrix function $q(\xi, \eta)$ of (2.3) satisfies the inequality

$$
\|\partial q(\xi, \eta) / \partial \eta\|+\left\|\nabla_{\xi} q(\xi, \eta)\right\|+\left\|\nabla_{\xi} \nabla_{\xi}^{*} q(\xi, \eta)\right\| \leq C \Lambda, \quad \xi \in \mathbf{R}^{d}, 0<\Re \eta<\Lambda,
$$

for some constant $C$. If $(\Omega, \mathcal{F}, P)$ is the i.i.d. environment, then $C$ depends only on $d, \delta, \Lambda$. If $(\Omega, \mathcal{F}, P)$ is the massive field theory environment, then $C$ additionally depends on $m$ and $\|D \tilde{\mathbf{a}}(\cdot)\|_{\infty}$. Furthermore each of the functions on the LHS of (A.1) are Hölder continuous. Thus if $g(\xi, \eta)$ is either of the functions $\partial q(\xi, \eta) / \partial \eta$ or $\nabla_{\xi} \nabla_{\xi}^{*} q(\xi, \eta)$, then there exists $\alpha>0$ depending only on $d, \delta$ such that

$$
\left\|g\left(\xi^{\prime}, \eta^{\prime}\right)-g(\xi, \eta)\right\| \leq C \Lambda\left[\left|\xi^{\prime}-\xi\right|^{\alpha}+\left|\left(\eta^{\prime}-\eta\right) / \Lambda\right|^{\alpha / 2}\right]
$$

for all $\xi^{\prime}, \xi \in \mathbf{R}^{d}, 0<\Re \eta^{\prime}, \Re \eta<\Lambda$. The constant $C$ in (A.2) has the same dependency as the constant $C$ in (A.1).

Proof. We just consider the case of the i.i.d. environment, since the proof for the field theory environment is similar. It is easy to see from the argument of Proposition 4.1 that $\|\partial q(\xi, \eta) / \partial \eta\| \leq C$. To see this note that in the representation (4.12) 
for $\partial q(\xi, \eta) / \partial \eta$ the function $h$ of (4.16) is in $L^{p}\left(\mathbf{Z}^{d+1}\right)$ for $p>(d+2) /(d+1)$. Since we can choose $p<2$, the uniform boundedness of $\partial q(\xi, \eta) / \partial \eta$, provided $\lambda / \Lambda$ is sufficiently close to 1 , follows from the generalized Jones' theorem [18]; see Corollary 5.1 .

We can bound the derivatives of $q(\xi, \eta)$ with respect to $\xi$ similarly. To bound $\nabla_{\xi} q(\xi, \eta)$ we can argue as in Lemma 3.1. We see from (3.6) that the function $g_{j}(x, t)$ corresponding to $\partial q(\xi, \eta) / \partial \xi_{j}$ is given by the formula

$$
g_{j}(x, t)=\Lambda\left[\nabla \nabla^{*} G_{\Lambda}(x, t-1)\right]^{*} e^{-\eta t-i x \cdot \xi} x_{j},
$$

which is in $L^{p}\left(\mathbf{Z}^{d+1}\right)$ for $p>(d+2) /(d+1)$. Hence $\partial q(\xi, \eta) / \partial \xi_{j}$ is uniformly bounded, provided $\lambda / \Lambda$ is sufficiently close to 1 , by the argument of the previous paragraph.

To bound $\nabla_{\xi} \nabla_{\xi}^{*} q(\xi, \eta)$ we note that we need to differentiate twice the series on the RHS of (2.14). The differentiated series can be written as a sum of two parts. The first part includes all terms where each gradient $\nabla_{\xi}$ and $\nabla_{\xi}^{*}$ acts on different operators $T_{\xi, \eta}$ on the RHS of (2.14), whereas the second part includes all terms where both gradients act on the same operator $T_{\xi, \eta}$. This in turn implies that a second derivative of $v_{1}^{*} q(\xi, \eta) v_{2}$ with $v_{1}, v_{2} \in \mathbf{C}^{d}$ is a sum $\Sigma_{1}+\Sigma_{2}$ with $\Sigma_{1}$ corresponding to the first part of the differentiated series (2.14). We therefore have as in (4.13) that

$$
\begin{aligned}
& \left(\frac{\partial^{2}}{\partial \xi_{j} \partial \xi_{k}}\right) v_{1}^{*} q(\xi, \eta) v_{2}=\Sigma_{1}+\Sigma_{2}, \quad \text { where } \\
& \Sigma_{2}=-\Lambda \sum_{r=1}^{100}\left[\tilde{T}_{1, \Im \xi, \Re \eta} g_{r}(\Re \xi, \Im \eta, \cdot) v_{1}, T_{1, \Im \xi, \Re \eta} h_{r}(\Re \xi, \Im \eta, \cdot) v_{2}\right]
\end{aligned}
$$

for any $d \times d$ matrix valued functions $g_{r}(x, t), h_{r}(x, t), x \in \mathbf{Z}^{d}, t \in \mathbf{Z}, r=1, \ldots, 100$ satisfying

(A.5)

$$
\sum_{r=1}^{100} \hat{g}_{r}(\zeta, \theta)^{*} \hat{h}_{r}(\zeta, \theta)=\left(\frac{\partial^{2}}{\partial \zeta_{j} \partial \zeta_{k}}\right) \frac{\Lambda e(i \Im \xi-\zeta) e(-i \Im \xi-\zeta)^{*}}{\left[e^{\Re \eta-i \theta}-1+\Lambda e(-i \Im \xi-\zeta)^{*} e(i \Im \xi-\zeta)\right]} .
$$

Since $\Sigma_{1}$ can be bounded by arguing as in the previous paragraph, it will be sufficient to show that the $g_{r}, h_{r}, r=1, \ldots, 100$ in (A.5) can be chosen so that they are in $L^{p}\left(\mathbf{Z}^{d+1}\right)$ for $p>(d+2) /(d+1)$. Observe that if one of the derivatives on the RHS of (A.5) is applied to the denominator and the other to the numerator, we are in a situation like (4.13), whence the corresponding functions $g_{r}, h_{r}$ on the LHS of A.5 can be chosen so that they are in $L^{p}\left(\mathbf{Z}^{d+1}\right)$ for $p>(d+2) /(d+1)$. If both derivatives on the RHS of $(\mathrm{A.5}$ ) are applied to the denominator, then the typical situation we are in is to find a factorization

$$
\hat{g}(\zeta, \theta)^{*} \hat{h}(\zeta, \theta)=\frac{\Lambda^{3} e_{r_{1}}(-\zeta) e_{r_{2}}(-\zeta) e_{r_{3}}(-\zeta) e_{r_{4}}(-\zeta)}{\left[e^{\Re \eta-i \theta}-1+\Lambda e(-\zeta)^{*} e(-\zeta)\right]^{3}}
$$

such that $g, h$ are both in $L^{p}\left(\mathbf{Z}^{d+1}\right)$ for $p>(d+2) /(d+1)$. Taking $\hat{g}(\zeta, \theta)$ to be given by the formula

$$
\hat{g}(\zeta, \theta)=\frac{\Lambda^{2} e_{r_{1}}(\zeta) e_{r_{2}}(\zeta) e_{r_{3}}(\zeta)}{\left[e^{\Re \eta-i \theta}-1+\Lambda e(-\zeta)^{*} e(-\zeta)\right]^{2}},
$$


it follows from (2.11) that $g(x, t), h(x, t)$ are the functions

$$
g(x, t)=t \nabla_{r_{1}}^{*} \nabla_{r_{2}}^{*} \nabla_{r_{3}}^{*} G(x, t-2), \quad h(x, t)=\Lambda \nabla_{r_{1}} G(x, t-1) .
$$

Now (2.12) implies that $h$ is in $L^{p}\left(\mathbf{Z}^{d+1}\right)$ for $p>(d+2) /(d+1)$, and by a similar argument we see that $g$ is also.

We have proved (A.1). The proof of (A.2) is similar.

Proposition A.1. Let $\delta$ be as in the statement of Lemma A.1. Then the inequality (1.12) of Theorem 1.2 holds with $\alpha=1$ if $1-\lambda / \Lambda \leq \delta$. If $q(0,0)$ is self-adjoint and $\nabla_{\xi} q(\xi, \eta)=0$ at $(\xi, \eta)=0$, then (1.12) holds with $\alpha=2$.

Proof. We first consider the discrete time case of (1.2). Similarly to (2.35) we have from (2.34) and Lemma 2.9, Lemma 2.10 of 8 , that

$$
\int_{[-\pi, \pi]^{d}}\left|\int_{-\pi / \varepsilon^{2}}^{\pi / \varepsilon^{2}} \frac{\varepsilon^{2}\left[\hat{f}_{\varepsilon}(\xi)-\hat{f}(\xi)\right] e^{-i \xi \cdot x+\eta\left(t+\varepsilon^{2}\right)}}{e^{\varepsilon^{2} \eta}-1+e(\varepsilon \xi)^{*} q\left(\varepsilon \xi, \varepsilon^{2} \eta\right) e(\varepsilon \xi)} d[\Im \eta]\right| d \xi \leq C \varepsilon^{2},
$$

for a constant $C$ depending only on the function $f(\cdot)$ and $d, \lambda, \Lambda$. We write $q(\xi, \eta)=$ $\tilde{q}(\xi, \tilde{\eta})$ where $\tilde{\eta}=e^{\eta}-1$ and use the Taylor inequality

$$
\left|\tilde{q}(\xi, \tilde{\eta})-\tilde{q}(\xi, 0)-\tilde{\eta} \frac{\partial \tilde{q}(\xi, 0)}{\partial \tilde{\eta}}\right| \leq \frac{|\tilde{\eta}|^{1+\alpha}}{1+\alpha} \sup _{0 \leq s \leq 1}\left|\frac{1}{|s \tilde{\eta}|^{\alpha}}\left[\frac{\partial \tilde{q}(\xi, s \tilde{\eta})}{\partial \tilde{\eta}}-\frac{\partial \tilde{q}(\xi, 0)}{\partial \tilde{\eta}}\right]\right| .
$$

Since

$$
\frac{\partial \tilde{q}(\xi, \tilde{\eta})}{\partial \tilde{\eta}}-\frac{\partial \tilde{q}(\xi, 0)}{\partial \tilde{\eta}}=\left(e^{-\eta}-1\right) \frac{\partial q(\xi, \eta)}{\partial \eta}+\left[\frac{\partial q(\xi, \eta)}{\partial \eta}-\frac{\partial q(\xi, 0)}{\partial \eta}\right],
$$

it follows from Lemma A1 and (A.10), A.11) that

$$
\left|q(\xi, \eta)-q(\xi, 0)-\left(e^{\eta}-1\right) \frac{\partial q(\xi, 0)}{\partial \eta}\right| \leq C\left|e^{\eta}-1\right|^{1+\alpha}, \quad \xi \in \mathbf{R}^{d}, 0<\Re \eta<\Lambda,
$$

where $\alpha>0$ is as in (A.2). Hence we have that

$$
\begin{aligned}
e^{\varepsilon^{2} \eta}- & 1+e(\varepsilon \xi)^{*} q\left(\varepsilon \xi, \varepsilon^{2} \eta\right) e(\varepsilon \xi)=|e(\varepsilon \xi)|^{2}\left|e^{\varepsilon^{2} \eta}-1\right|^{1+\alpha} \operatorname{Error}\left(\varepsilon \xi, \varepsilon^{2} \eta\right) \\
+ & \left\{1+e(\varepsilon \xi)^{*}[\partial q(\varepsilon \xi, 0) / \partial \eta] e(\varepsilon \xi)\right\}\left(e^{\varepsilon^{2} \eta}-1\right)+e(\varepsilon \xi)^{*} q(\varepsilon \xi, 0) e(\varepsilon \xi)
\end{aligned}
$$

where by A.12 the function $\operatorname{Error}(\xi, \eta)$ is uniformly bounded for $\xi \in \mathbf{R}^{d}, 0<$ $\Re \eta<\Lambda$. It follows from Corollary 2.2, Lemma A1 and A.13 that there are positive constants $\nu, C_{1}, C_{2}$ such that

$$
\begin{aligned}
& \text { (A.14) } \mid \frac{1}{2 \pi} \int_{-\pi / \varepsilon^{2}}^{\pi / \varepsilon^{2}} \frac{\varepsilon^{2} e^{\eta\left(t+\varepsilon^{2}\right)}}{e^{\varepsilon^{2} \eta}-1+e(\varepsilon \xi)^{*} q\left(\varepsilon \xi, \varepsilon^{2} \eta\right) e(\varepsilon \xi)} d[\Im \eta] \\
& -\frac{1}{2 \pi} \int_{-\pi / \varepsilon^{2}}^{\pi / \varepsilon^{2}} \frac{\varepsilon^{2} e^{\eta\left(t+\varepsilon^{2}\right)}}{\left\{1+e(\varepsilon \xi)^{*}[\partial q(\varepsilon \xi, 0) / \partial \eta] e(\varepsilon \xi)\right\}\left(e^{\varepsilon^{2} \eta}-1\right)+e(\varepsilon \xi)^{*} q(\varepsilon \xi, 0) e(\varepsilon \xi)} d[\Im \eta] \mid \\
& \leq C_{1} \int_{-\pi / \varepsilon^{2}}^{\pi / \varepsilon^{2}} \frac{|\xi|^{2}\left|\mathrm{e}^{\varepsilon^{2} \eta}-1\right|^{1+\alpha}}{\left(|\eta|+\Lambda|\xi|^{2}\right)^{2}} d[\Im \eta] \leq C_{2}(\varepsilon|\xi|)^{2} \quad \text { if } \varepsilon|\xi| \leq \nu .
\end{aligned}
$$


We can restate (A.14) similarly to (2.38) as

$$
\begin{aligned}
& \mid \frac{1}{2 \pi} \int_{-\pi / \varepsilon^{2}}^{\pi / \varepsilon^{2}} \frac{\varepsilon^{2} e^{\eta\left(t+\varepsilon^{2}\right)}}{e^{\varepsilon^{2} \eta}-1+e(\varepsilon \xi)^{*} q\left(\varepsilon \xi, \varepsilon^{2} \eta\right) e(\varepsilon \xi)} d[\Im \eta] \\
& \quad-\frac{1}{1+e(\varepsilon \xi)^{*}[\partial q(\varepsilon \xi, 0) / \partial \eta] e(\varepsilon \xi)}\left[1-\frac{e(\varepsilon \xi)^{*} q(\varepsilon \xi, 0) e(\varepsilon \xi)}{1+e(\varepsilon \xi)^{*}[\partial q(\varepsilon \xi, 0) / \partial \eta] e(\varepsilon \xi)}\right]^{t / \varepsilon^{2}} \mid \\
& \quad \leq C_{2}(\varepsilon|\xi|)^{2} .
\end{aligned}
$$

Estimating integrals with respect to $\xi$ for $\varepsilon|\xi|>\nu$ as in (2.35) we conclude from (A.15) that

$$
\begin{aligned}
& \left|\left\langle u_{\varepsilon}\left(x / \varepsilon, t / \varepsilon^{2}, \cdot\right)\right\rangle-u_{\mathrm{hom}}(x, t)\right| \\
& \quad \leq C_{4} \varepsilon^{2}+\int_{\varepsilon|\xi|<\nu} d \xi|\hat{f}(\xi)| \exp \left[-\left\{\xi^{*} q(0,0) \xi\right\} t\right] \\
& \quad-\frac{1}{1+e(\varepsilon \xi)^{*}[\partial q(\varepsilon \xi, 0) / \partial \eta] e(\varepsilon \xi)}\left[1-\frac{e(\varepsilon \xi)^{*} q(\varepsilon \xi, 0) e(\varepsilon \xi)}{1+e(\varepsilon \xi)^{*}[\partial q(\varepsilon \xi, 0) / \partial \eta] e(\varepsilon \xi)}\right]^{t / \varepsilon^{2}} \mid,
\end{aligned}
$$

for some constant $C_{4}$. It follows from Lemma A1 upon using the interpolation identity

$$
e^{b}-e^{a}=(b-a) \int_{0}^{1} e^{\rho b+(1-\rho) a} d \rho
$$

that there is a constant $C_{5}$ such that the integral on the RHS of (A.16) is bounded by $C_{5} \varepsilon$ in general and by $C_{5} \varepsilon^{2}$ if $q(0,0)$ is self-adjoint and $\nabla_{\xi} q(0,0)=0$. We have therefore proved the proposition in the discrete time case.

The argument for the continuous time case is similar. Instead of Lemma 2.9, Lemma 2.10 of [8] we use Lemma 5.4, Lemma 5.5 of [8]. The main point to note is that integrals with respect to $\Im \eta$ over regions $|\Im \eta|>\pi / \varepsilon^{2}$ are $O\left(\varepsilon^{2}\right)$.

Remark 8. The real matrix $q(0,0)$ is self-adjoint if the environment is timeindependent; i.e. $\tau_{0, t}=$ identity for all $t \in \mathbf{Z}$ or $t \in \mathbf{R}$. In general it is not self-adjoint; see Lemma 2.6 of [8].

There does not appear to be any general criteria which would imply that $q(0,0)$ is self-adjoint and $\nabla_{\xi} q(0,0)=0$ for the PDE (1.2), (1.4) with $\nabla$ defined by the forward difference (1.5). However if we define $\nabla$ by the central difference in which

$$
\nabla_{i} \phi(x)=\frac{1}{2}\left[\phi\left(x+\mathbf{e}_{i}\right)-\phi\left(x-\mathbf{e}_{i}\right)\right], \quad \nabla_{i}^{*}=-\nabla_{i}, \quad i=1, \ldots, d,
$$

then there are such criteria. First we note that for the PDE (1.2), 1.4) with $\nabla$ defined by (A.18) the identities (2.2), (2.3), (2.5) hold if instead of (2.1) we set

$$
\begin{aligned}
\partial_{j, \xi} \psi(\omega) & =\frac{1}{2}\left[e^{-i \mathbf{e}_{j} \cdot \xi} \psi\left(\tau_{\mathbf{e}_{j}, 0} \omega\right)-e^{i \mathbf{e}_{j} \cdot \xi} \psi\left(\tau_{-\mathbf{e}_{j}, 0} \omega\right)\right] \\
e_{j}(\xi) & =\frac{1}{2}\left[e^{-i \mathbf{e}_{j} \cdot \xi}-e^{i \mathbf{e}_{j} \cdot \xi}\right], \quad j=1, \ldots, d .
\end{aligned}
$$


It follows from (A.20) that there is a constant $C$ depending only on $d$ such that

$$
\left\|\varepsilon^{-2} e(\varepsilon \xi)^{*} q(0,0) e(\varepsilon \xi)-\xi^{*} q(0,0) \xi\right\| \leq C \varepsilon^{2}|\xi|^{4}, \quad \xi \in[-\pi, \pi]^{d} .
$$

Hence if $\nabla$ is defined by the central difference (A.18) the argument of Proposition A1 and (A.21) imply that we can take $\alpha=2$ in (1.12) provided $\nabla_{\xi} q(0,0)=0$ and $\lambda / \Lambda$ is sufficiently close to 1 .

We show that $\nabla_{\xi} q(0,0)=0$ if the environment $(\Omega, \mathcal{F}, P)$ is reflection invariant. To define reflection invariance of $\Omega$ first denote by $R_{i}: \mathbf{Z}^{d} \rightarrow \mathbf{Z}^{d}, i=1, \ldots, d$, the reflections on $\mathbf{Z}^{d}$ which are the linear maps determined by the relations $R_{i} \mathbf{e}_{j}=$ $\mathbf{e}_{j}-2 \delta(i-j) \mathbf{e}_{i}, j=1, \ldots, d$. We say that $(\Omega, \mathcal{F}, P)$ is reflection invariant if there exist mappings $\tilde{R}_{i}: \Omega \rightarrow \Omega, i=1, \ldots, d$, which are measure preserving involutions $\left(\tilde{R}_{i}^{2}=\right.$ identity) and $\tau_{x, t} \tilde{R}_{i}=\tilde{R}_{i} \tau_{R_{i} x, t}$ for $x \in \mathbf{Z}^{d}$ and $t$ in $\mathbf{Z}$ or $\mathbf{R}$.

Lemma A.2. Assume $(\Omega, \mathcal{F}, P)$ is a reflection invariant environment, a $(\cdot)$ is a diagonal matrix and $\mathbf{a}\left(\tilde{R}_{k} \omega\right)=\mathbf{a}(\omega), \omega \in \Omega, k=1, . ., d$. If $\nabla$ is defined by the central difference (A.18) and $q(\cdot, \cdot)$ is the function defined by (2.3), then

$$
q\left(R_{k} \xi, \eta\right)=q(\xi, \eta) \text { for } \xi \in \mathbf{R}^{d}, \Re \eta>0, k=1, \ldots, d .
$$

Proof. Observe from A.19) that $\partial_{j, \xi}^{*}=-\partial_{j, \xi}, j=1, \ldots, d, \xi \in \mathbf{R}^{d}$. We also have that $\tilde{R}_{k} \partial_{j, \xi}=\partial_{j, R_{k} \xi} \tilde{R}_{k}$ if $k \neq j$ and $\tilde{R}_{k} \partial_{j, \xi}=-\partial_{j, R_{k} \xi} \tilde{R}_{k}$ if $k=j$. It follows by applying $\tilde{R}_{k}$ to (2.2) that if $\mathbf{a}(\cdot)$ is a diagonal matrix, then

$$
\Phi_{j}\left(\xi, \eta, \tilde{R}_{k} \omega\right)=[1-2 \delta(k-j)] \Phi_{j}\left(R_{k} \xi, \eta, \omega\right), \quad \xi \in \mathbf{R}^{d}, \omega \in \Omega .
$$

We conclude from A.23) that if $\Psi(\xi, \eta, \omega)=\partial_{\xi} \Phi(\xi, \eta, \omega)$, then the $d \times d$ matrix $\Psi(\xi, \eta, \omega)=\left[\Psi_{i, j}(\xi, \eta, \omega)\right]$ has the property

$$
\Psi_{j, j}\left(\xi, \eta, \tilde{R}_{k} \omega\right)=\Psi_{j, j}\left(R_{k} \xi, \eta, \omega\right), \quad \xi \in \mathbf{R}^{d}, \omega \in \Omega, j=1, \ldots, d .
$$

Now (A.22) follows from (2.3) and A.24 on using the fact that $\tilde{R}_{k}$ is measure preserving on $\Omega$.

In the case of an environment $(\Omega, \mathcal{F}, P)$ for which $\Omega$ is a set of mappings $\omega$ : $\mathbf{Z}^{d} \times \mathcal{T} \rightarrow \mathbf{R}^{n}$ with $\mathcal{T}=\mathbf{Z}$ or $\mathbf{R}$, we can define $\tilde{R}_{k}$ by $\tilde{R}_{k} \omega(x, t)=\omega\left(R_{k} x, t\right), x \in$ $\mathbf{Z}^{d}, t \in \mathcal{T}$. Both the i.i.d. and massive field theory environments of Theorem 1.2 are of this nature. In the i.i.d. case $n=d(d+1) / 2$ and $\omega(x, t)=\mathbf{a}(x, t), x \in \mathbf{Z}^{d}, t \in \mathbf{Z}$, with $P$ being a product measure on $\Omega$. In the field theory case $\omega(x, t)=\phi(x, t), x \in$ $\mathbf{Z}^{d}, t \in \mathbf{R}$. The mapping $\tilde{R}_{k}$ is clearly measure preserving in the i.i.d. case, but is only measure preserving in the massive field theory case if $V\left(R_{k} z\right)=V(z), z \in \mathbf{R}^{d}$. Hence the i.i.d. environment is always reflection invariant, and the field theory environment is reflection invariant provided the function $V(z), z \in \mathbf{R}^{d}$, is reflection invariant.

\section{ACKNOWLEDGEMENTS}

The authors would like to thank Tom Spencer for helpful conversations. They would also like to thank the referee for helpful comments on the original manuscript. 


\section{REFERENCES}

[1] C. Boldrighini, R. A. Minlos, and A. Pellegrinotti, Random walks in quenched i.i.d. space-time random environment are always a.s. diffusive, Probab. Theory Related Fields 129 (2004), no. 1, 133-156, DOI 10.1007/s00440-003-0331-x. MR2052866 (2005e:60048)

[2] Herm Jan Brascamp and Elliott H. Lieb, On extensions of the Brunn-Minkowski and Prékopa-Leindler theorems, including inequalities for log concave functions, and with an application to the diffusion equation, J. Functional Analysis 22 (1976), no. 4, 366-389. MR0450480 (56 \#8774)

[3] Leo Breiman, Probability, Classics in Applied Mathematics, vol. 7, Society for Industrial and Applied Mathematics (SIAM), Philadelphia, PA, 1992. Corrected reprint of the 1968 original. MR:1163370 (93d:60001)

[4] Luis A. Caffarelli and Panagiotis E. Souganidis, Rates of convergence for the homogenization of fully nonlinear uniformly elliptic pde in random media, Invent. Math. 180 (2010), no. 2, 301-360, DOI 10.1007/s00222-009-0230-6. MR2609244 (2011c:35041)

[5] A. P. Calderon and A. Zygmund, On the existence of certain singular integrals, Acta Math. 88 (1952), 85-139. MR0052553 (14,637f)

[6] G. A. Chechkin, A. L. Piatnitski, and A. S. Shamaev, Homogenization, Methods and applications, Translations of Mathematical Monographs, vol. 234, American Mathematical Society, Providence, RI, 2007. Translated from the 2007 Russian original by Tamara Rozhkovskaya. MR2337848 (2008j:35013)

[7] Joseph G. Conlon, PDE with random coefficients and Euclidean field theory, J. Statist. Phys. 116 (2004), no. 1-4, 933-958, DOI 10.1023/B:JOSS.0000037204.93858.f2. MR2082201 (2005e:81132)

[8] Joseph G. Conlon, Green's functions for elliptic and parabolic equations with random coefficients. II, Trans. Amer. Math. Soc. 356 (2004), no. 10, 4085-4142 (electronic), DOI 10.1090/S0002-9947-04-03467-1. MR.2058840 (2005f:35341)

[9] Joseph G. Conlon and Ali Naddaf, On homogenization of elliptic equations with random coefficients, Electron. J. Probab. 5 (2000), no. 9, 58 pp. (electronic), DOI 10.1214/EJP.v565. MR1768843 (2002j:35328)

[10] Joseph G. Conlon and Thomas Spencer, Strong convergence to the homogenized limit of elliptic equations with random coefficients, Trans. Amer. Math. Soc. 366 (2014), no. 3, $1257-$ 1288, DOI 10.1090/S0002-9947-2013-05762-5. MR3145731

[11] T. Delmotte and J.-D. Deuschel, On estimating the derivatives of symmetric diffusions in stationary random environment, with applications to $\nabla \phi$ interface model, Probab. Theory Related Fields 133 (2005), no. 3, 358-390, DOI 10.1007/s00440-005-0430-y. MR2198017 (2007a:60057)

[12] Dmitry Dolgopyat, Gerhard Keller, and Carlangelo Liverani, Random walk in Markovian environment, Ann. Probab. 36 (2008), no. 5, 1676-1710, DOI 10.1214/07-AOP369. MR2440920 (2009f:60124)

[13] Richard Durrett, Probability: theory and examples, 2nd ed., Duxbury Press, Belmont, CA, 1996. MR1609153 (98m:60001)

[14] T. Funaki and H. Spohn, Motion by mean curvature from the Ginzburg-Landau $\nabla \phi$ interface model, Comm. Math. Phys. 185 (1997), no. 1, 1-36, DOI 10.1007/s002200050080. MR 1463032 (98f:60206)

[15] Antoine Gloria and Felix Otto, An optimal variance estimate in stochastic homogenization of discrete elliptic equations, Ann. Probab. 39 (2011), no. 3, 779-856, DOI 10.1214/10-AOP571. MR2789576(2012j:35018)

[16] Mathieu Gourcy and Liming Wu, Logarithmic Sobolev inequalities of diffusions for the $L^{2}$ metric, Potential Anal. 25 (2006), no. 1, 77-102, DOI 10.1007/s11118-006-9009-1. MR:2238937(2008h:60214)

[17] Bernard Helffer and Johannes Sjöstrand, On the correlation for Kac-like models in the convex case, J. Statist. Phys. 74 (1994), no. 1-2, 349-409, DOI 10.1007/BF02186817. MR1257821 (95g:82022)

[18] B. Frank Jones Jr., A class of singular integrals, Amer. J. Math. 86 (1964), 441-462. MR0161099 (28 \#4308)

[19] S. M. Kozlov, Averaging of random structures (Russian), Dokl. Akad. Nauk SSSR 241 (1978), no. 5, 1016-1019. MR510894 (80e:60078) 
[20] S. M. Kozlov, The averaging method and walks in inhomogeneous environments (Russian), Uspekhi Mat. Nauk 40 (1985), no. 2(242), 61-120, 238. MR786087(87b:60104)

[21] C. Landim, S. Olla, and H. T. Yau, Convection-diffusion equation with space-time ergodic random flow, Probab. Theory Related Fields 112 (1998), no. 2, 203-220, DOI 10.1007/s004400050187. MR 1653837 (99j:35084)

[22] Per-Gunnar Martinsson and Gregory J. Rodin, Asymptotic expansions of lattice Green's functions, R. Soc. Lond. Proc. Ser. A Math. Phys. Eng. Sci. 458 (2002), no. 2027, 26092622, DOI 10.1098/rspa.2002.0985. MR1942800(2003j:82019)

[23] Jean-Christophe Mourrat, Kantorovich distance in the martingale CLT and quantitative homogenization of parabolic equations with random coefficients, Probab. Theory Related Fields 160 (2014), no. 1-2, 279-314, DOI 10.1007/s00440-013-0529-5. MR3256815

[24] A. Naddaf and T. Spencer, Estimates on the variance of some homogenization problems, 1998 preprint.

[25] David Nualart, The Malliavin calculus and related topics, 2nd ed., Probability and its Applications (New York), Springer-Verlag, Berlin, 2006. MR2200233 (2006j:60004)

[26] G. C. Papanicolaou and S. R. S. Varadhan, Boundary value problems with rapidly oscillating random coefficients, Random fields, Vol. I, II (Esztergom, 1979), Colloq. Math. Soc. János Bolyai, vol. 27, North-Holland, Amsterdam, 1981, pp. 835-873. MR712714 (84k:58233)

[27] William Parry, Topics in ergodic theory, Cambridge Tracts in Mathematics, vol. 75, Cambridge University Press, Cambridge, 2004. Reprint of the 1981 original. MR2140546 (2005m:37003)

[28] Rémi Rhodes, On homogenization of space-time dependent and degenerate random flows, Stochastic Process. Appl. 117 (2007), no. 10, 1561-1585, DOI 10.1016/j.spa.2007.01.010. MR2353040 (2008j:60195)

[29] Michael Reed and Barry Simon, Methods of modern mathematical physics. II. Fourier analysis, self-adjointness, Academic Press [Harcourt Brace Jovanovich Publishers], New York, 1975. MR0493420 (58 \#12429b)

[30] Elias M. Stein, Singular integrals and differentiability properties of functions, Princeton Mathematical Series, No. 30, Princeton University Press, Princeton, N.J., 1970. MR0290095 (44 \#7280)

[31] Elias M. Stein and Guido Weiss, Introduction to Fourier analysis on Euclidean spaces, Princeton Mathematical Series, No. 32, Princeton University Press, Princeton, N.J., 1971. MR0304972 (46 \#4102)

[32] V. V. Yurinski i, Averaging of symmetric diffusion in a random medium (Russian), Sibirsk. Mat. Zh. 27 (1986), no. 4, 167-180, 215. MR867870(88e:35190)

[33] V. V. Jikov, S. M. Kozlov, and O. A. Ole inik, Homogenization of differential operators and integral functionals, Springer-Verlag, Berlin, 1994. Translated from the Russian by G. A. Yosifian [G. A. Iosif'yan]. MR1329546(96h:35003b) 1109

Department of Mathematics, University of Michigan, Ann Arbor, Michigan 48109-

E-mail address: conlon@umich.edu

Department of Mathematics, University of Michigan, Ann Arbor, Michigan 481091109

E-mail address: fahimara@umich.edu

Current address: Department of Mathematics, Florida State University, Tallahassee, Florida 32306

E-mail address: fahim@math.fsu.edu 